Review

\title{
Quantum Entanglement Concentration Based on Nonlinear Optics for Quantum Communications
}

\author{
Yu-Bo Sheng ${ }^{1,2, *}$ and Lan Zhou ${ }^{2,3}$ \\ ${ }^{1}$ Institute of Signal Processing Transmission, Nanjing University of Posts and Telecommunications, \\ Nanjing 210003, China \\ ${ }^{2}$ Key Lab of Broadband Wireless Communication and Sensor Network Technology, Nanjing \\ University of Posts and Telecommunications, Ministry of Education, Nanjing 210003, China \\ ${ }^{3}$ College of Mathematics \& Physics, Nanjing University of Posts and Telecommunications, Nanjing \\ 210003, China; E-Mail: zhoul@ njupt.edu.cn (L.Z.) \\ * Author to whom correspondence should be addressed; E-Mail: shengyb@ njupt.edu.cn; \\ Tel./Fax: +86-025-83492417.
}

Received: 14 March 2013; in revised form: 3 May 2013 / Accepted: 8 May 2013 /

Published: 16 May 2013

\begin{abstract}
Entanglement concentration is of most importance in long distance quantum communication and quantum computation. It is to distill maximally entangled states from pure partially entangled states based on the local operation and classical communication. In this review, we will mainly describe two kinds of entanglement concentration protocols. One is to concentrate the partially entangled Bell-state, and the other is to concentrate the partially entangled W state. Some protocols are feasible in current experimental conditions and suitable for the optical, electric and quantum-dot and optical microcavity systems.
\end{abstract}

Keywords: quantum entanglement; entanglement concentration; quantum communication

\section{Introduction}

Quantum communication and quantum computation have attracted much attention over the last 20 years, due to the absolute safety in the information transmission for quantum communication and the super fast factoring for quantum computation [1,2]. However, in most of the communication protocols, such as quantum teleportation [3], quantum key distribution (QKD) [4-6], quantum secure 
direct communication (QSDC) [7-9] and quantum secret sharing [10-12], the basic requirement is to set up the quantum entanglement channel via entanglement distribution. Unfortunately, the entanglement decreases exponentially with the length of the connecting channel, because of the optical absorption and inevitable channel noise.

In this way, quantum repeaters are used [13-16]. Moreover, in order to obtain a high quality of entanglement for long distance communication, the entanglement distillation is required. Usually, the entanglement distillation mainly includes three different classes $[17,18]$. The first one is the entanglement purification that focuses on the general mixed state [19-29]. Entanglement purification is to distill highly entangled states from mixed states. The second one is the Procrustean method. The third one is the Schmidt decomposition method. These two methods also are called the entanglement concentration [17,18,30-37]. In optical fiber transmission, the dielectric constant acquires a temporal and spatial dependence. Therefore, if the time delay between the photons is small, the effect of the noise is known as the unitary collective noise model. Some entanglement distribution protocols based on collective noise are proposed [38-41]. Entanglement concentration is to distill maximally entangled states from pure partially entangled states. It has been used as a basis for theoretically-oriented results in quantum information and gives operational meaning to the von Neumann entropy [42]. The Schmidt decomposition method is a powerful way for realizing the entanglement concentration. The first entanglement concentration protocol (ECP) is proposed by Bennett et al. using collective measurement [30]. Using linear optical elements, Zhao et al. and Yamamoto et al. developed the Schmidt decomposition method and proposed two similar ECPs, respectively [33,34]. Two independent experiments were reported for linear optical entanglement concentration [35,36].

Most ECPs are based on linear optics, for the photons can be manipulated and controlled easily. However, in current quantum communication and computation, linear optics has an inherent defect, because it is usually based on the post-selection principle. After the photons are detected by the single photon detectors, the photons are destroyed simultaneously, and they cannot be further used. Using nonlinear optics, such as the cross-Kerr nonlinearity, or other systems, as the auxiliary, can greatly improve such protocols.

In this review, we will mainly describe some ECPs based on the nonlinear optical elements. The review is organized as follows: In Section 2, we describe the ECPs with Bell states. In Section 3, we explain the ECPs for W states. In Section 4, we mainly describe some ECPs for NOON states, ECPs for electrons and quantum dot and optical microcavities systems. In Section 5, we will provide a discussion and make a conclusion.

\section{ECPs for Bell States}

Cross-Kerr nonlinearity provides us with a good tool to construct nondestructive quantum nondemolition detectors (QND), which have the potential for conditioning the evolution of our system without necessarily destroying the single photon. The Hamiltonian of a cross-Kerr nonlinear medium can be written by the form [43-45]:

$$
H_{Q N D}=\hbar \chi \hat{n}_{a} \hat{n}_{c}
$$


where $\hat{n}_{a}\left(\hat{n}_{c}\right)$ denotes the number operator for mode a (c) and $\hbar \chi$ is the coupling strength of the nonlinearity, which is decided by the property of material. For instance, for a signal photon state, $|\varphi\rangle=a|0\rangle+b|1\rangle$, and a coherent state, $|\alpha\rangle$, the cross-Kerr interaction causes the combined system composed of a single photon and a coherent state to evolve as [43]:

$$
U_{c k}|\varphi\rangle|\alpha\rangle=e^{i H_{Q N D} t / \hbar}(a|0\rangle+b|1\rangle)|\alpha\rangle=a|0\rangle|\alpha\rangle+b|1\rangle\left|\alpha e^{i \theta}\right\rangle
$$

Here, the $|0\rangle$ and $|1\rangle$ are the number of the photons. After the interaction, the signal photon state is unaffected, but the coherent probe beam $|\alpha\rangle$ makes a phase shift of $\theta$ with $\theta=\chi t, t$ is the interaction time here. One can see that the phase shift is directly proportional to the number of photons.

Figure 1 is the basic construction of the parity-check gate, which was first proposed by Nemoto and Munro in 2004 [43]. It is also a powerful element in current quantum information processing, such as Bell state analysis [46-48], entanglement purification [22-24], and so on [49-52]. Let us suppose that two polarization qubits, $a 1$ and $a 2$, are initially in the state:

$$
\left|\Psi_{a 1}\right\rangle=\alpha_{1}|H\rangle+\alpha_{2}|V\rangle
$$

and

$$
\left|\Psi_{a 2}\right\rangle=\beta_{1}|H\rangle+\beta_{2}|V\rangle
$$

$|H\rangle$ and $|V\rangle$ are the polarization state of the photon, with horizontal and vertical polarization, respectively.

Figure 1. The parity-check gate was constructed by Nemoto and Munro [43].

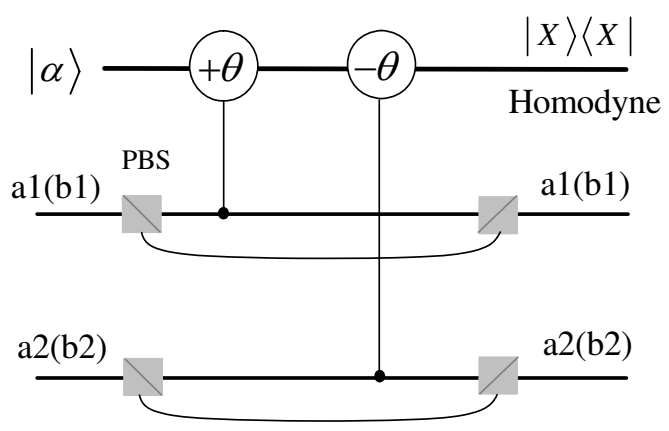

These two qubits are transmitted into the spatial modes, $a_{1}$ and $a_{2}$, respectively, and they interact with cross-Kerr nonlinearities. The polarization beam splitter (PBS) transmits the $|H\rangle$ polarization photon and reflects the $|V\rangle$ polarization photon. The action of the PBS's and cross-Kerr nonlinearities will make the whole state of the two photons evolve to:

$$
\left|\Psi_{T}\right\rangle_{a b}=\alpha_{1} \beta_{1}|H H\rangle_{a b}|\alpha\rangle+\alpha_{1} \beta_{2}|H V\rangle_{a b}\left|\alpha e^{i \theta}\right\rangle+\alpha_{2} \beta_{1}|V H\rangle_{a b}\left|\alpha e^{-i \theta}\right\rangle+\alpha_{2} \beta_{2}|V V\rangle_{a b}|\alpha\rangle
$$

One can observe immediately that the items, $|H H\rangle$ and $|V V\rangle$, make the coherent state pick up no phase shift and remain as a coherent state with respect to each other. However, the items, $|H V\rangle$ and $|V H\rangle$, pick up phase shifts, $\theta$ and $-\theta$, respectively. The different phase shifts can be distinguished by a 
general homodyne-heterodyne measurement. We can also choose the local oscillator phase, $\pi / 2$, offset from the probe phase, which can be used to make states, $\left|\alpha e^{ \pm \theta}\right\rangle$, undistinguishable [43]. As mentioned in [43], it is called an $X$ quadrature measurement $(|X\rangle\langle X|)$, as shown in Figure 1. The operation of the QND measurement relies on distinguishing the phase shift of the coherent state. However, the coherent states are not orthogonal; this will lead to the effect of errors and imperfection. The probability of this error occurring is given by $P_{\text {error }}=\frac{1}{2}\left(1-\operatorname{Erf}\left[X_{d} / 2 \sqrt{2}\right]\right)$, and it is less than $10^{-5}$ when the distance is $X_{d} \sim \alpha \theta^{2}>9$ [43]. Here, $X_{d}=2 \alpha[1-\cos \theta]$. This requirement can be satisfied with the large amplitude of the coherent state. Therefore, this requirement may be feasible with current experimental technology. If we choose to call the $|H H\rangle$ and $|V V\rangle$ even parity states and $|H V\rangle$ and $|V H\rangle$ odd parity states, the action of the parity-check gate is very clear: it splits the even parity states deterministically from the odd parity states by nondemolition measurements. If we let the initial coefficient, $\alpha_{1}=\beta_{1}=\frac{1}{\sqrt{2}}$, one can easily generate four Bell states, with the same probability of $1 / 4$. The four Bell states are:

$$
\begin{aligned}
& \left|\Phi^{ \pm}\right\rangle=\frac{1}{\sqrt{2}}(|H H\rangle \pm|V V\rangle) \\
& \left|\Psi^{ \pm}\right\rangle=\frac{1}{\sqrt{2}}(|H V\rangle \pm|V H\rangle)
\end{aligned}
$$

\subsection{ECP with Cross-Kerr Nonlinearity}

In this section, we will start to describe the ECPs based on nonlinear optics, say cross-Kerr nonlinearity. The three ECPs are also shown in [53-55].

From Figure 2, suppose there are two identical photon pairs with less entanglement, $a_{1} b_{1}$ and $a_{2} b_{2}$. The photons, $a$, belong to Alice, and photons, $b$, belong to Bob. The photon pairs, $a_{1} b_{1}$ and $a_{2} b_{2}$, are in the following unknown polarization entangled states [53]:

$$
\begin{aligned}
& |\Phi\rangle_{a_{1} b_{1}}=\alpha|H\rangle_{a_{1}}|H\rangle_{b_{1}}+\beta|V\rangle_{a_{1}}|V\rangle_{b_{1}} \\
& |\Phi\rangle_{a_{2} b_{2}}=\alpha|H\rangle_{a_{2}}|H\rangle_{b_{2}}+\beta|V\rangle_{a_{2}}|V\rangle_{b_{2}}
\end{aligned}
$$

where $|\alpha|^{2}+|\beta|^{2}=1$.

Figure 2. Schematic diagram of the proposed entanglement concentration protocol (ECP) [53]. Two pairs of identical less entanglement photons are sent to Alice and Bob from source $1\left(S_{1}\right)$ and source $2\left(S_{2}\right)$. The QND is a parity-checking device shown in Figure 3. The wave plates, $R_{45}$ and $R_{90}$, rotate the horizontal and vertical polarizations by $45^{\circ}$ and $90^{\circ}$, respectively.

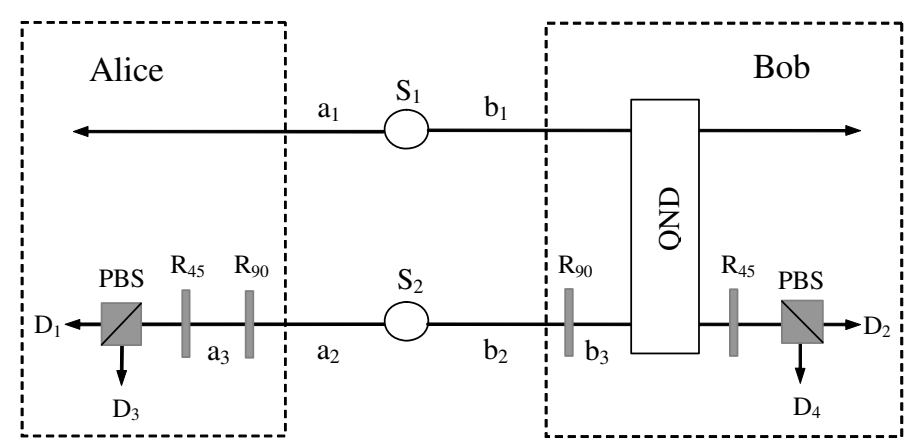


Figure 3. The parity check gate with only one Kerr material $[45,53,56]$. The original work is shown in [56], with a difference in that the probe beam is rotated by $\theta$.

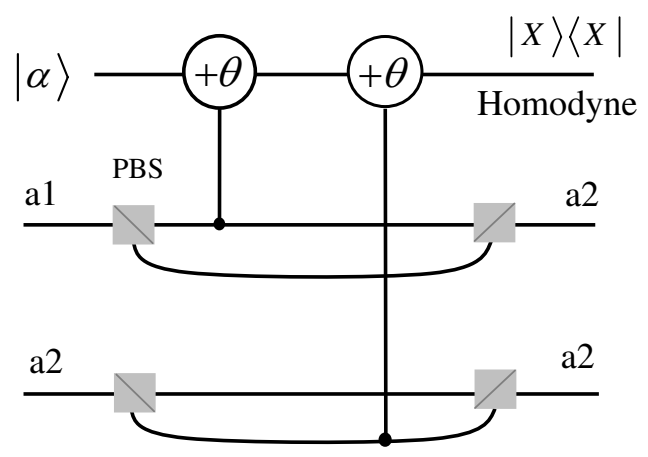

The two parties, Alice and Bob, first rotate the polarization states of the second photons by $90^{\circ}$ with half-wave plates (i.e., $R_{90}$ shown in Figure 2), the state of the four photons can evolve to:

$$
\begin{aligned}
|\Psi\rangle^{\prime} & =\alpha^{2}|H\rangle_{a_{1}}|V\rangle_{a_{3}}|H\rangle_{b_{1}}|V\rangle_{b_{3}}+\alpha \beta|H\rangle_{a_{1}}|H\rangle_{a_{3}}|H\rangle_{b_{1}}|H\rangle_{b_{3}} \\
& +\alpha \beta|V\rangle_{a_{1}}|V\rangle_{a_{3}}|V\rangle_{b_{1}}|V\rangle_{b_{3}}+\beta^{2}|V\rangle_{a_{1}}|H\rangle_{a_{3}}|V\rangle_{b_{1}}|H\rangle_{b_{3}}
\end{aligned}
$$

Here, $a_{3}\left(b_{3}\right)$ is used to label the photon, $a_{2}\left(b_{2}\right)$, after the half-wave plate $R_{90}$.

From Equation (8), it is obvious that items, $|H\rangle_{a_{1}}|H\rangle_{a_{3}}|H\rangle_{b_{1}}|H\rangle_{b_{3}}$ and $|V\rangle_{a_{1}}|V\rangle_{a_{3}}|V\rangle_{b_{1}}|V\rangle_{b_{3}}$, have the same coefficient of $\alpha \beta$, but the other two terms are different. After the two photons pass through the QND, Bob may get three different kinds of phase shifts: $\theta, 2 \theta$ and 0 . The phase shift, $\theta$, corresponds to the items, $|H H\rangle$ and $|V V\rangle$. The phase shift, 0, corresponds to the item, $|V H\rangle$, and $2 \theta$ corresponds to $|H V\rangle$. We first discuss the case that the phase shift is $\theta$. The state of the photons becomes

$$
|\Psi\rangle^{\prime \prime}=\frac{1}{\sqrt{2}}\left(|H\rangle_{a_{1}}|H\rangle_{a_{3}}|H\rangle_{b_{1}}|H\rangle_{b_{3}}+|V\rangle_{a_{1}}|V\rangle_{a_{3}}|V\rangle_{b_{1}}|V\rangle_{b_{3}}\right)
$$

The probability that Alice and Bob get the above state is: $P_{s_{1}}=2|\alpha \beta|^{2}$.

Now, both pairs, $a_{1} b_{1}$ and $a_{3} b_{3}$, are in the same polarization. Alice and Bob use their $\lambda / 4$-wave plates, $R_{45}$, to rotate the photons, $a_{3}$ and $b_{3}$, by $45^{\circ}$. After the rotations, Equation (9) will evolve into:

$$
\begin{aligned}
|\Psi\rangle^{\prime \prime \prime} & =\frac{1}{2 \sqrt{2}}\left(|H\rangle_{a 1}|H\rangle_{b 1}+|V\rangle_{a 1}|V\rangle_{b 1}\right)\left(|H\rangle_{a 3}|H\rangle_{b 3}+|V\rangle_{a 3}|V\rangle_{b 3}\right) \\
& +\frac{1}{2 \sqrt{2}}\left(|H\rangle_{a 1}|H\rangle_{b 1}-|V\rangle_{a 1}|V\rangle_{b 1}\right)\left(|H\rangle_{a 3}|V\rangle_{b 3}+|V\rangle_{a 3}|H\rangle_{b 3}\right)
\end{aligned}
$$

From Equation (10), we need to distinguish the photons in modes, $a_{3}$ and $b_{3}$. One can see that if the two detectors, $D_{1}$ and $D_{2}$, or the two detectors, $D_{3}$ and $D_{4}$, fire, the photon pair, $a_{1} b_{1}$, is left in the state:

$$
\left|\phi^{+}\right\rangle_{a_{1} b_{1}}=\frac{1}{\sqrt{2}}\left(|H\rangle_{a_{1}}|H\rangle_{b_{1}}+|V\rangle_{a_{1}}|V\rangle_{b_{1}}\right)
$$

If $D_{1}$ and $D_{4}$ or $D_{2}$ and $D_{3}$ fire, the photon pair, $a_{1} b_{1}$, is left in the state:

$$
\left|\phi^{-}\right\rangle_{a_{1} b_{1}}=\frac{1}{\sqrt{2}}\left(|H\rangle_{a_{1}}|H\rangle_{b_{1}}-|V\rangle_{a_{1}}|V\rangle_{b_{1}}\right)
$$


Both $\left|\phi^{+}\right\rangle_{a_{1} b_{1}}$ and $\left|\phi^{-}\right\rangle_{a_{1} b_{1}}$ are the maximally entangled states. A bit-flip operation is needed to convert $\left|\phi^{-}\right\rangle_{a_{1} b_{1}}$ to $\left|\phi^{+}\right\rangle_{a_{1} b_{1}}$.

In fact, in this protocol, the QND essentially acts as the role of parity check. It picks up the two photons in the same polarization state, i.e., $|H H\rangle$ and $|V V\rangle$, with the same phase shift of $\theta$. This ECP does not require sophisticated single-photon detectors and can reach the same efficiency as the conventional concentration protocol. Interestingly, the efficiency of this ECP can be improved in the next step, because in the above description, we only consider the case that both of the parties choose the phase shift, $\theta$. If we choose $\theta=\pi$, in this way, $2 \theta$ and 0 represent the same phase shift, 0 . Therefore, if both of the parties get the phase shift 0 , the initial state will collapse to:

$$
\left|\Phi_{1}\right\rangle^{\prime \prime}=\alpha^{2}|H\rangle_{a_{1}}|V\rangle_{a_{3}}|H\rangle_{b_{1}}|V\rangle_{b_{3}}+\beta^{2}|V\rangle_{a_{1}}|H\rangle_{a_{3}}|V\rangle_{b_{1}}|H\rangle_{b_{3}}
$$

The above state is essentially the nonmaximally entangled state and can be concentrated again. In detail, Alice and Bob take a rotation of $90^{\circ}$ on each photon of the second four-photon system and make the state of this system become:

$$
\left|\Phi_{2}\right\rangle^{\prime \prime}=\beta^{2}|H\rangle_{a_{1}^{\prime}}|V\rangle_{a_{3}^{\prime}}|H\rangle_{b_{1}^{\prime}}|V\rangle_{b_{3}^{\prime}}+\alpha^{2}|V\rangle_{a_{1}^{\prime}}|H\rangle_{a_{3}^{\prime}}|V\rangle_{b_{1}^{\prime}}|H\rangle_{b_{3}^{\prime}}
$$

The state of the composite system composed of eight photons becomes:

$$
\begin{aligned}
& \left|\Phi_{s}\right\rangle^{\prime \prime} \equiv\left|\Phi_{1}\right\rangle^{\prime \prime} \otimes\left|\Phi_{2}\right\rangle^{\prime \prime}=\alpha^{2} \beta^{2}\left(|H\rangle_{a_{1}}|V\rangle_{a_{3}}|H\rangle_{b_{1}}|V\rangle_{b_{3}}|H\rangle_{a_{1}^{\prime}}|V\rangle_{a_{3}^{\prime}}|H\rangle_{b_{1}^{\prime}}|V\rangle_{b_{3}^{\prime}}\right. \\
+ & \left.|V\rangle_{a_{1}}|H\rangle_{a_{3}}|V\rangle_{b_{1}}|H\rangle_{b_{3}}|V\rangle_{a_{1}^{\prime}}|H\rangle_{a_{3}^{\prime}}|V\rangle_{b_{1}^{\prime}}|H\rangle_{b_{3}^{\prime}}\right)+\alpha^{4}|H\rangle_{a_{1}}|V\rangle_{a_{3}}|H\rangle_{b_{1}}|V\rangle_{b_{3}}|V\rangle_{a_{1}^{\prime}}|H\rangle_{a_{3}^{\prime}}|V\rangle_{b_{1}^{\prime}}|H\rangle_{b_{3}^{\prime}} \\
+ & \beta^{4}|V\rangle_{a_{1}}|H\rangle_{a_{3}}|V\rangle_{b_{1}}|H\rangle_{b_{3}}|H\rangle_{a_{1}^{\prime}}|V\rangle_{a_{3}^{\prime}}|H\rangle_{b_{1}^{\prime}}|V\rangle_{b_{3}^{\prime}}
\end{aligned}
$$

The first two terms have the same coefficient, $\alpha^{2} \beta^{2}$, and can be picked upped by detecting the two photons, $b_{3}$ and $b_{3}^{\prime}$, with the QND. If Bob gets the phase shift, $\theta=\pi$, the eight photons will collapse to the state:

$$
\begin{aligned}
\left|\Phi_{s}\right\rangle^{\prime \prime \prime} & =\frac{1}{\sqrt{2}}\left(|H\rangle_{a_{1}}|V\rangle_{a_{3}}|H\rangle_{b_{1}}|V\rangle_{b_{3}}|H\rangle_{a_{1}^{\prime}}|V\rangle_{a_{3}^{\prime}}|H\rangle_{b_{1}^{\prime}}|V\rangle_{b_{3}^{\prime}}\right. \\
& \left.+|V\rangle_{a_{1}}|H\rangle_{a_{3}}|V\rangle_{b_{1}}|H\rangle_{b_{3}}|V\rangle_{a_{1}^{\prime}}|H\rangle_{a_{3}^{\prime}}|V\rangle_{b_{1}^{\prime}}|H\rangle_{b_{3}^{\prime}}\right)
\end{aligned}
$$

The probability that Alice and Bob get this state is:

$$
P_{s_{2}}=\frac{2|\alpha \beta|^{4}}{\left(|\alpha|^{4}+|\beta|^{4}\right)^{2}}
$$

They have the probability, $P_{f_{2}}^{\prime}=1-P_{s_{2}}$, to obtain the partially entangled state:

$$
\begin{aligned}
\left|\Phi_{1}\right\rangle^{\prime \prime \prime} & =\alpha^{4}|H\rangle_{a_{1}}|V\rangle_{a_{3}}|H\rangle_{b_{1}}|V\rangle_{b_{3}}|V\rangle_{a_{1}^{\prime}}|H\rangle_{a_{3}^{\prime}}|V\rangle_{b_{1}^{\prime}}|H\rangle_{b_{3}^{\prime}} \\
& +\beta^{4}|V\rangle_{a_{1}}|H\rangle_{a_{3}}|V\rangle_{b_{1}}|H\rangle_{b_{3}}|H\rangle_{a_{1}^{\prime}}|V\rangle_{a_{3}^{\prime}}|H\rangle_{b_{1}^{\prime}}|V\rangle_{b_{3}^{\prime}}
\end{aligned}
$$

which can be concentrated by iteration of the process discussed above.

For the eight photons in the state described by Equation (16), Alice and Bob can obtain a maximally entangled photon pair with some single-photon measurements on the other six photons. 
We can denote the yield of the maximally entangled state, $Y$, as $|\alpha \beta|^{2}$. The yield is the ratio of the number of maximally entangled photon pairs, $N_{m}$, and the number of originally partially entangled photon pairs, $N_{l}$. With the iteration of the entanglement concentration process, the yield of this ECP is:

$$
Y=\sum_{i=1}^{n} Y_{i}
$$

where:

$$
\begin{aligned}
& Y_{1}=|\alpha \beta|^{2} \\
& Y_{2}= \frac{1}{2}\left(1-2|\alpha \beta|^{2}\right) \frac{|\alpha \beta|^{4}}{\left(|\alpha|^{4}+|\beta|^{4}\right)^{2}} \\
& Y_{3}=\frac{1}{2^{2}}\left(1-2|\alpha \beta|^{2}\right)\left[1-\frac{|\alpha \beta|^{4}}{\left(|\alpha|^{4}+|\beta|^{4}\right)^{2}}\right] \frac{|\alpha \beta|^{8}}{\left(|\alpha|^{8}+|\beta|^{8}\right)^{2}} \\
& Y_{n}=\frac{1}{2^{n-1}}\left(\prod_{j=2}^{n-1}\left[1-\frac{2|\alpha \beta|^{2^{j-1}}}{\left(|\alpha|^{2^{j-1}}+|\beta|^{2^{j-1}}\right)^{2}}\right]\right) \frac{|\alpha \beta|^{2^{n}}}{\left(|\alpha|^{2^{n}}+|\beta|^{2^{n}}\right)^{2}}
\end{aligned}
$$

The yield is shown in Figure 4 with the change of the iteration number of entanglement concentration processes, $n$, and the coefficient, $\alpha \in[0,1]$.

One can easily obtain the success probability:

$$
P_{s_{n}}=\frac{2|\alpha \beta|^{2^{n}}}{\left(|\alpha|^{2^{n}}+|\beta|^{2^{n}}\right)^{2}}
$$

where $n$ is the iteration number of the entanglement concentration processes. The $Y_{n}$ can also be rewritten as:

$$
Y_{n}=\frac{1}{2^{n}}\left(\prod_{j=2}^{n-1}\left[1-P_{s_{j-1}}\right]\right) P_{s_{n}}
$$

Figure 4. The yield $(Y)$ is altered with the iteration number of entanglement concentration processes, $n$, and the coefficient, $\alpha \in[0,1]$ [53].

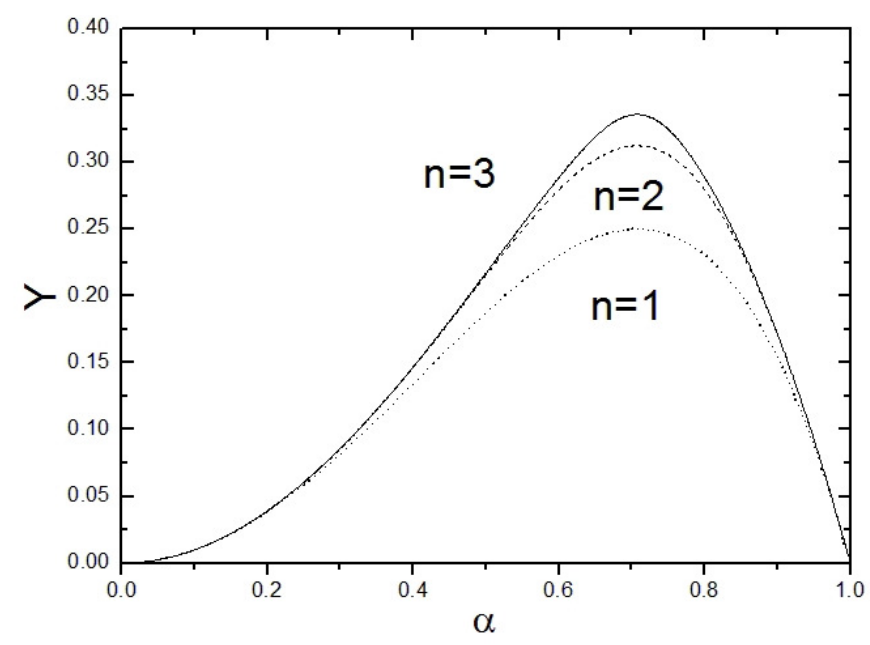


It is straightforward to generalize this ECP to distill the maximally entangled multipartite Greenberger-Horne-Zeilinger (GHZ) state states from the partially entangled GHZ-class states. $\mathrm{N}$-particle GHZ-class states can be described as:

$$
\left|\Phi^{\prime+}\right\rangle=\alpha|H H \cdots H\rangle+\beta|V V \cdots V\rangle
$$

Figure 5 shows the principle of this ECP for multipartite GHZ-class states. Two pairs of N-particles are sent to each party, say, Alice, Bob, Charlie, etc., and each party gets two photons. Following the same principle described above, they can ultimately obtain the maximally entangled GHZ state with the same success probability described above.

Figure 5. Schematic diagram of the multipartite entanglement concentration scheme [53]. $2 N$ photons in two partially entangled $N$-particle Greenberger-Horne-Zeilinger (GHZ)-class states are sent to $N$ parties of quantum communication-say, Alice, Bob, Charlie, etc. Photons 2 and $N+2$ are sent to Bob and enter the quantum nondemolition detectors (QND) to complete a parity-check measurement. After the QND measurement, Bob asks the others to retain their photons if his two photons have the same parity, $(|H H\rangle$ or $|V V\rangle)$, and remove them for the next iteration if Bob gets an odd parity $(|H V\rangle$ or $|V H\rangle)$.

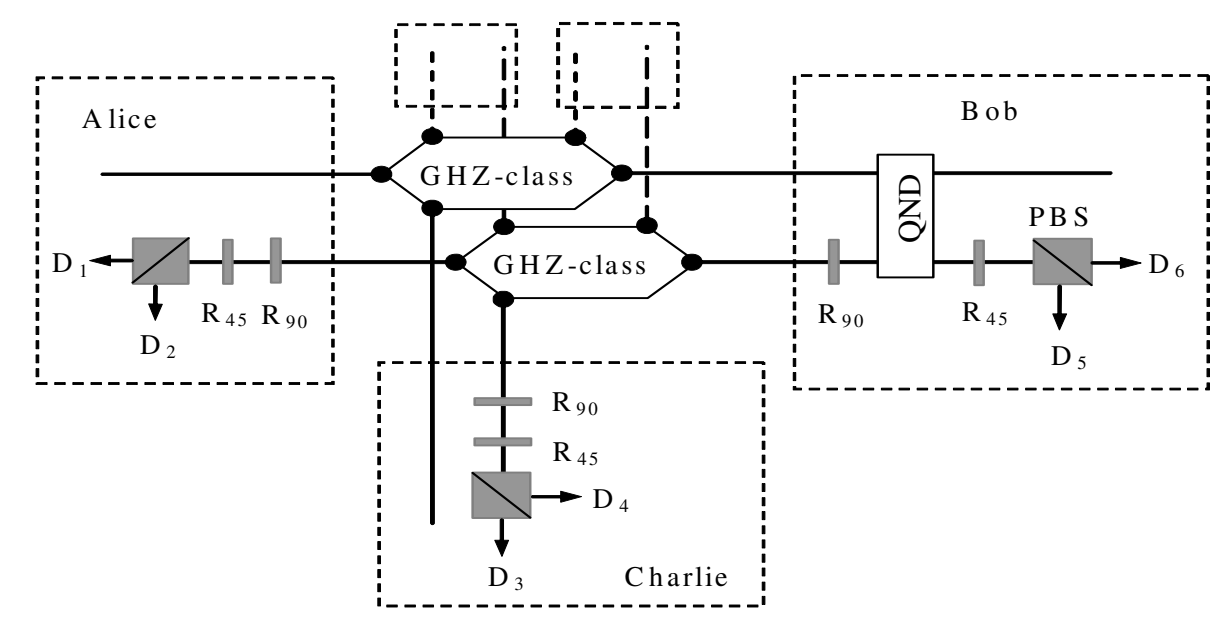

\subsection{Single-Photon-Assisted Entanglement Concentration}

In the above section, we described the ECP with cross-Kerr nonlinearity. Compared with the ECPs with linear optics [33,34], it can be repeated to obtain a higher success probability. Moreover, the concentrated maximally entangled state can be retained, while in linear optics, it is always destroyed with the post-selection principle. However, it is still not the optimal one. It is shown that the two copies of partially entangled states are not necessary. One pair of partially entangled states and a single photon can also complete the task [54]. In this section, we will describe two ECPs both assisted with single photons. The first one uses linear optics, and the second one uses the cross-Kerr nonlinearity. From Figure 6, suppose the partially entangled pair of photons emitted from $S_{1}$ are sent to Alice and Bob. The photon, $a$, belongs to Alice, and $b$ belongs to Bob. The initial photon pair is in the following state:

$$
|\Phi\rangle_{a 1 b 1}=\alpha|H\rangle_{a 1}|H\rangle_{b 1}+\beta|V\rangle_{a 1}|V\rangle_{b 1}
$$


Figure 6. A schematic drawing of the single-photon-assisted ECP with linear optics [54]. $S_{1}$ is the partial entanglement source, and $S_{2}$ is the single photon source. $\mathrm{HWP}_{90}$ and $\mathrm{HWP}_{45}$ can rotate the polarization of the state by $90^{\circ}$ and $45^{\circ}$, respectively.

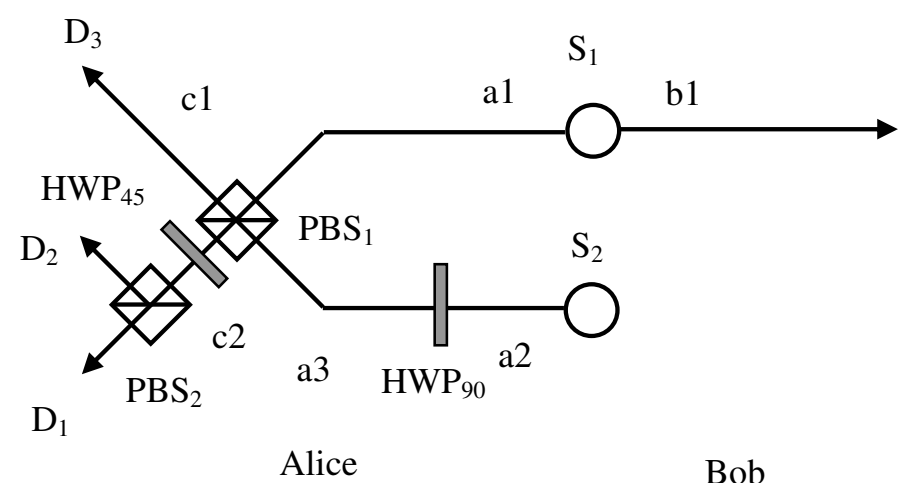

Suppose that we know the coefficients, $\alpha$ and $\beta$. In a practical experiment, one can measure enough samples to obtain the information of the state. Another source, $S_{2}$, emits a single photon with the form:

$$
|\Phi\rangle_{a 2}=\alpha|H\rangle_{a 2}+\beta|V\rangle_{a 2}
$$

$a 1, b 1$ and $a 2$ are different spatial modes. Before the photons pass through the PBSs, Alice first rotates the polarization state of the single photon, $|\Phi\rangle_{a 2}$, by $90^{\circ}$ by the half-wave plate $\left(\mathrm{HWP}_{90}\right.$ in Figure 6). Then, the whole system can be rewritten as:

$$
\begin{aligned}
|\Psi\rangle^{\prime} & =|\Phi\rangle_{a 1 b 1} \otimes\left|\Phi^{\prime}\right\rangle_{a 3} \\
& =\alpha^{2}|H\rangle_{a 1}|H\rangle_{b 1}|V\rangle_{a 3}+\alpha \beta|H\rangle_{a 1}|H\rangle_{b 1}|H\rangle_{a 3}+\alpha \beta|V\rangle_{a 1}|V\rangle_{b 1}|V\rangle_{a 3}+\beta^{2}|V\rangle_{a 1}|V\rangle_{b 1}|H\rangle_{a 3} \\
& =\alpha^{2}|H\rangle_{a 1}|V\rangle_{a 3}|H\rangle_{b 1}+\beta^{2}|V\rangle_{a 1}|H\rangle_{a 3}|V\rangle_{b 1}+\alpha \beta\left(|H\rangle_{a 1}|H\rangle_{a 3}|H\rangle_{b 1}+|V\rangle_{a 1}|V\rangle_{a 3}|V\rangle_{b 1}\right)
\end{aligned}
$$

From the above equation, it is obvious that the items, $|H\rangle_{a 1}|H\rangle_{a 3}|H\rangle_{b 1}$ and $|V\rangle_{a 1}|V\rangle_{a 3}|V\rangle_{b 1}$, will lead the two output modes, $c 1$ and $c 2$, both exactly containing only one photon. However, item $|H\rangle_{a 1}|V\rangle_{a 3}|H\rangle_{b 1}$ will lead two photons both in $c 2$ mode, and item $|V\rangle_{a 1}|H\rangle_{a 3}|V\rangle_{b 1}$ will lead both photons in $c 1$ mode. Therefore, by choosing the three-mode cases, i.e., each mode of $c 1, c 2$ and $b 1$ exactly contains one photon, and the initial state can be projected into a maximally three-photon entangled state:

$$
|\Psi\rangle^{\prime \prime}=\frac{1}{\sqrt{2}}\left(|H\rangle_{c 1}|H\rangle_{c 2}|H\rangle_{b 1}+|V\rangle_{c 1}|V\rangle_{c 2}|V\rangle_{b 1}\right)
$$

with a probability of $2|\alpha \beta|^{2}$. In order to obtain a maximally entangled state, they could perform a $45^{\circ}$ polarization measurement on the photon in mode $c 2$. In Figure 6, with the quarter-wave plate $\left(\mathrm{HWP}_{45}\right)$, Equation (27) will evolve to:

$$
|\Psi\rangle^{\prime \prime \prime}=\frac{1}{2}\left(|H\rangle_{c 1}|H\rangle_{b 1}+|V\rangle_{c 1}|V\rangle_{b 1}\right)|H\rangle_{c 2}+\left(|H\rangle_{c 1}|H\rangle_{b 1}-|V\rangle_{c 1}|V\rangle_{b 1}\right)|V\rangle_{c 2}
$$

Now, Alice lets the photon, $c 2$, pass through the $\mathrm{PBS}_{2}$. Clearly, if the detector, $D_{1}$, fires, the photon pair will be left in the state as:

$$
\left|\phi^{+}\right\rangle_{a 1 b 1}=\frac{1}{\sqrt{2}}\left(|H\rangle_{c 1}|H\rangle_{b 1}+|V\rangle_{c 1}|V\rangle_{b 1}\right)
$$


If the detector, $D_{2}$, fires, the photon pair will be left in the state as:

$$
\left|\phi^{-}\right\rangle_{a 1 b 1}=\frac{1}{\sqrt{2}}\left(|H\rangle_{c 1}|H\rangle_{b 1}-|V\rangle_{c 1}|V\rangle_{b 1}\right)
$$

Both Equations (29) and (30) are the maximally entangled states. One of them, say Alice or Bob, only needs to perform a phase flip to convert Equation (30) to Equation (29), and the whole concentration process is finished.

The above ECP can also be improved with QND. As shown in Figure 7, we use the QND to substitute the PBS. The whole system, $|\Phi\rangle_{a 1 b 1} \otimes\left|\Phi^{\prime}\right\rangle_{a 3}$, with the coherent state, $|\alpha\rangle$, can be rewritten:

$$
\begin{aligned}
& |\Psi\rangle^{\prime}|\alpha\rangle=|\Phi\rangle_{a 1 b 1} \otimes\left|\Phi^{\prime}\right\rangle_{a 3}|\alpha\rangle \\
= & \left(\alpha^{2}|H\rangle_{a 1}|H\rangle_{b 1}|V\rangle_{a 3}+\alpha \beta|H\rangle_{a 1}|H\rangle_{b 1}|H\rangle_{a 3}+\alpha \beta|V\rangle_{a 1}|V\rangle_{b 1}|V\rangle_{a 3}+\beta^{2}|V\rangle_{a 1}|V\rangle_{b 1}|H\rangle_{a 3}\right)|\alpha\rangle \\
\rightarrow & \alpha^{2}|H\rangle_{a 1}|V\rangle_{a 3}|H\rangle_{b 1}\left|\alpha e^{i 2 \theta}\right\rangle+\beta^{2}|V\rangle_{a 1}|H\rangle_{a 3}|V\rangle_{b 1}|\alpha\rangle \\
+ & \alpha \beta\left(|H\rangle_{a 1}|H\rangle_{a 3}|H\rangle_{b 1}+|V\rangle_{a 1}|V\rangle_{a 3}|V\rangle_{b 1}\right)\left|\alpha e^{i \theta}\right\rangle
\end{aligned}
$$

With the same principle in Section 2, if the phase shift of homodyne measurement is $\theta$, Alice asks Bob to keep the whole state. Otherwise, they discard the state. The remaining state is essentially the state described in Equation (27). Therefore, following the same step described above, one can ultimately obtain the maximally entangled state $\left|\phi^{+}\right\rangle_{c 1 b 1}$ if $D_{1}$ fires, and get $\left|\phi^{-}\right\rangle_{c 1 b 1}$ if $D_{2}$ fires. Certainly, if a suitable cross-Kerr medium is available and Alice can control the interaction time, $t$, exactly, which makes the phase shift $\theta=\pi$, one cannot distinguish the phase shift 0 and $2 \pi$. The discarded items in the above equation can be written as:

$$
\left|\Phi^{\prime}\right\rangle=\alpha^{2}|H\rangle_{c 1}|V\rangle_{a 3}|H\rangle_{b 1}+\beta^{2}|V\rangle_{c 1}|H\rangle_{a 3}|V\rangle_{b 1}
$$

with the probability of $|\alpha|^{4}+|\beta|^{4}$. Alice uses the $\mathrm{HWP}_{45}$ to rotate the photon in $c 2$, and finally, it is detected by $D_{1}$ or $D_{2}$. Equation (32) will become:

$$
\left|\Phi^{\prime \prime}\right\rangle=\alpha^{2}|H\rangle_{c 1}|H\rangle_{b 1}+\beta^{2}|V\rangle_{c 1}|V\rangle_{b 1}
$$

if $D_{1}$ fires, and will become:

$$
\left|\Phi^{\prime \prime \prime}\right\rangle=\alpha^{2}|H\rangle_{c 1}|H\rangle_{b 1}-\beta^{2}|V\rangle_{c 1}|V\rangle_{b 1}
$$

if $D_{2}$ fires. With the same principle with Section 2.1, this ECP can also be repeated to obtain a higher success probability.

It is known that local operation and classical communication cannot increase entanglement. Entanglement concentration is essentially the transformation of entanglement. We define the entanglement transformation efficiency $\eta$ as:

$$
\eta=\frac{E_{c}}{E_{0}}
$$

Here, $E_{0}$ is the entanglement of an initially partial entangled state, and $E_{c}$ is the entanglement of the state after performing the concentration process one time. $E_{c}$ can be described as:

$$
E_{c}=P_{s} \times 1+\left(1-P_{s}\right) \times E^{\prime}
$$


$E^{\prime}$ is the entanglement of the remaining state if the concentration is unsuccessful. For a two-body pure entangled state, von Neumann entropy is suitable to describe the entanglement. The entanglement of the initial state in Equation (24) can be described as:

$$
E_{0}=-|\alpha|^{2} \log _{2}|\alpha|^{2}-|\beta|^{2} \log _{2}|\beta|^{2}
$$

Figure 7. The schematic drawing of the ECP with QND [54]. Here, we substitute the polarization beam splitter (PBS) shown in Figure 6 with QND. The QND is described in Figure 3. This ECP can obtain a higher success probability.

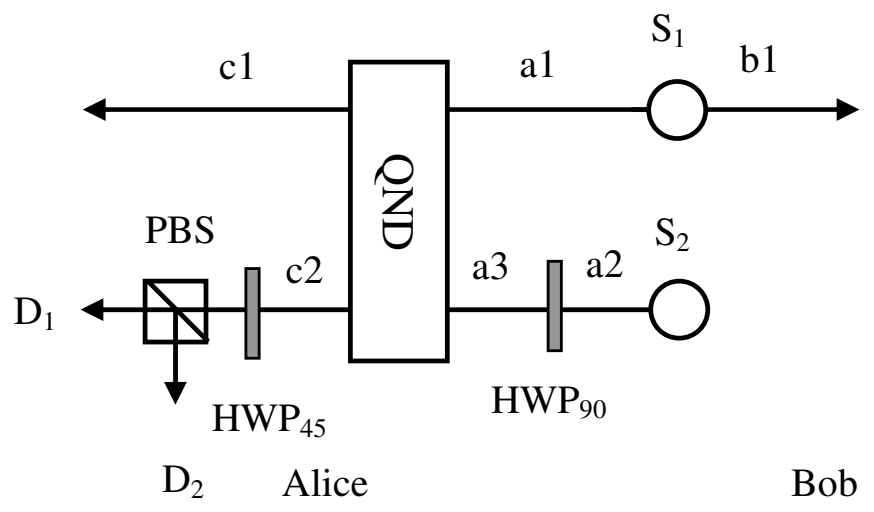

We can calculate the $\eta$ in each previous ECP. We call [33] as the PBS1 protocol, Ref [53] as the QND1 protocol, the first protocol of this section as PBS2 protocol and the second protocol as the QND2 protocol. The $\eta$ of PBS1 protocol is [33]:

$$
\eta_{P B S 1}=\frac{2|\alpha \beta|^{2} \times 1}{2 E}=\frac{|\alpha \beta|}{E}
$$

For the ECP in Section 2.1 [53]:

$$
\eta_{Q N D 1}=\frac{E_{Q N D 1}^{\prime}}{2 E}
$$

with:

$$
\begin{aligned}
E_{Q N D 1}^{\prime} & =2|\alpha \beta|^{2}+\left(|\alpha|^{4}+|\beta|^{4}\right)\left[-\frac{|\alpha|^{4}}{|\alpha|^{4}+|\beta|^{4}} \log _{2} \frac{|\alpha|^{4}}{|\alpha|^{4}+|\beta|^{4}}\right. \\
& \left.-\frac{|\beta|^{4}}{|\alpha|^{4}+|\beta|^{4}} \log _{2} \frac{|\beta|^{4}}{|\alpha|^{4}+|\beta|^{4}}\right]
\end{aligned}
$$

In this ECP, we obtain:

$$
\eta_{P B S 2}=\frac{2|\alpha \beta|^{2}}{E}=2 \eta_{P B S 1}
$$

and in QND2 protocol:

$$
\eta_{Q N D 2}=\frac{E_{Q N D 1}^{\prime}}{E}=2 \eta_{Q N D 1}
$$


From Figure 8, it is shown that the $\eta$ increases with the initial entanglement and can reach the max value, 1, in the QND2 protocol. In Figure 9, we also calculate the limit of entanglement transformation efficiency by iterating the protocol $N(N \rightarrow \infty)$ times.

$$
\eta_{Q N D 2}^{N \rightarrow \infty}=\frac{\sum_{N=1}^{\infty} E_{N} P_{N}}{E_{0}}=\frac{P}{E_{0}}
$$

Obviously, $\eta$ is monotone, increasing with the entanglement of the initial state, and can get the max value, 1 , when the initial state is a maximally entangled one, that is, $\alpha=\frac{1}{\sqrt{2}}$. However, in the PBS1 protocol, it only reaches 0.25 , and they both reach 0.5 in the QND1 protocol and PBS2 protocol. Therefore, only the QND2 protocol can completely distill the entanglement, theoretically.

Figure 8. The entanglement transformation efficiency, $\eta$, is altered with the coefficient, $\alpha$, after performing each protocol one time [54]. Curves B, C, D and E correspond to the protocols of PBS1 [33], QND1 [53], PBS2 and QND2, respectively. All the curves show that $\eta$ increases with the entanglement of the initial entangled state. The QND2 protocol has the highest transformation efficiency. It can reach maximum value, 1 , when $\alpha=\frac{1}{\sqrt{2}}$.

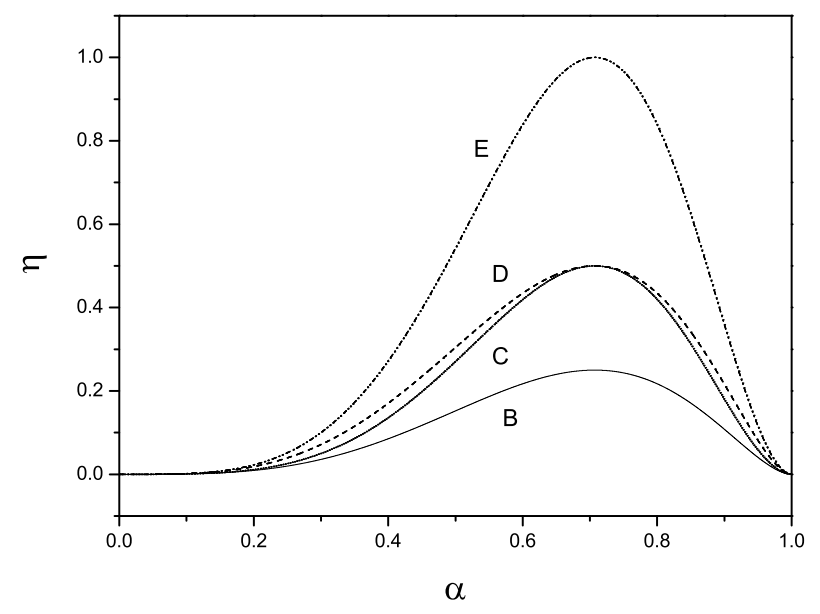

Figure 9. The entanglement transformation efficiency, $\eta$, plotted against $\alpha$ after performing each protocol $N$ times $(N \rightarrow \infty)$ in the QND2 protocol. For numerical simulation, we let $N=10$ as a good approximation [54].

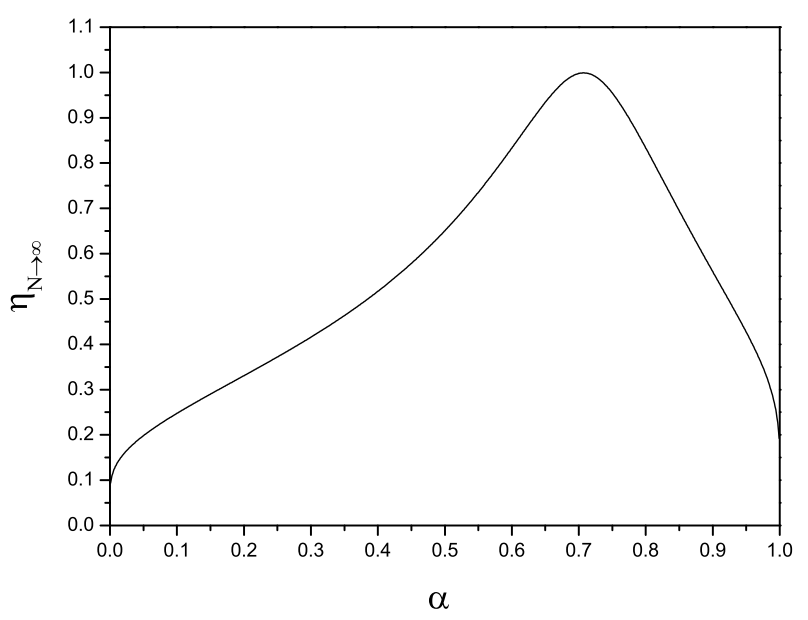


This ECP is quite different from the ECP described in Section 2.1. In each round, we only require one pair of the partially entangled state and can reach the same success probability as before. This makes it optimal. Moreover, only one of the parties needs to operate the ECP. It is more useful when used to concentrate the multipartite GHZ state. Therefore, this ECP is more suitable than the ECP in Section 2.1.

\subsection{Entanglement Concentration for Single-Photon Entanglement}

Single-photon entanglement may be the simplest entanglement with the form of $\frac{1}{\sqrt{2}}\left(|1\rangle_{A}|0\rangle_{B}+\right.$ $\left.|0\rangle_{A}|1\rangle_{B}\right)=\frac{1}{\sqrt{2}}\left(a^{\dagger}+b^{\dagger}\right)|0\rangle$. It is a superposition state in location A or B. Here, the $|0\rangle$ and $|1\rangle$ represent the photon numbers, 0 and 1 , respectively. The most important application for single-photon entanglement may be the quantum repeater protocol in long-distance quantum communication [13]. We take the famous Duan-Lukin-Cirac-Zoller (DLCZ) protocol, for example [16]. The first step for the DLCZ protocol is to entangle two atomic ensembles in distance locations, A and B. The pair sources are coherently excited by synchronized classical pumping pulses, and then, they emit a photon pair with a small probability, $p / 2$, corresponding to the state $[16,55]$ :

$$
\left[1+\sqrt{\frac{p}{2}}\left(a^{\dagger} a^{\prime \dagger}+b^{\dagger} b^{\prime \dagger}\right)+o(p)\right]|0\rangle
$$

Here, $a^{+}\left(b^{+}, a^{\prime+}\right.$ or $\left.b^{+}\right)$is the creation operation for the mode, $a\left(b, a^{\prime}\right.$ or $\left.b^{\prime}\right)$. However, in a practical manipulation, we cannot ensure that the pair sources excited by the synchronized classical pumping pulses always have the same probability. For example, in location A, it may be in the following form:

$$
|0\rangle_{a}|0\rangle_{a^{\prime}}+\sqrt{\frac{p_{a}}{2}} a^{\dagger} a^{\prime \dagger}|0\rangle_{a}|0\rangle_{a^{\prime}}+o\left(p_{a}\right)
$$

but in location B, the pair source may emit a pair with the form:

$$
|0\rangle_{b}|0\rangle_{b^{\prime}}+\sqrt{\frac{p_{b}}{2}} b^{\dagger} b^{\dagger \dagger}|0\rangle_{b}|0\rangle_{b^{\prime}}+o\left(p_{b}\right)
$$

$\frac{p_{a}}{2}$ and $\frac{p_{b}}{2}$ are two different probabilities for locations A and B, respectively.

The whole system evolves as:

$$
\left[1+\sqrt{\frac{p_{a}}{2}} a^{\dagger} a^{\prime \dagger}|0\rangle_{a}|0\rangle_{a^{\prime}}+\sqrt{\frac{p_{b}}{2}} b^{\dagger} b^{\prime \dagger}|0\rangle_{b}|0\rangle_{b^{\prime}}+o(p)\right]|0\rangle
$$

Finally, after the detection of the photon by a 50:50 beam splitter (BS), the single-photon entangled state will become: $\left(\sqrt{\frac{p_{a}}{2}} a^{\dagger}+\sqrt{\frac{p_{b}}{2}} e^{i \theta_{A B}} b^{\dagger}\right)|0\rangle$. We can rewrite it as:

$$
\left|\Psi^{\prime}\right\rangle_{a b}=\left(\alpha a^{\dagger}+\beta e^{i \theta_{A B}} b^{\dagger}\right)|0\rangle
$$

where $|\alpha|^{2}+|\beta|^{2}=1 . \theta_{A B}$ is the relative phase between $\mathrm{A}$ and $\mathrm{B}$.

Equation (48) is the entanglement of photonic modes, and we can convert it to the memory modes with $M_{A}$ and $M_{B}$. In a quantum repeater protocol, after entanglement generation, they need to extend the entanglement to long distance with entanglement swapping for long-distance communication. If the entanglement between $M_{A}$ and $M_{B}$ and $M_{C}$ and $M_{D}$ are both maximally entangled ones, we can 
easily establish the maximal entanglement between $M_{A}$ and $M_{D}$ [16]. However, if we cannot get the maximally entangled states during entanglement generation, but get the entanglement as Equation (48), then the combination of $\left|\Psi^{\prime}\right\rangle_{a b}$ and $\left|\Psi^{\prime}\right\rangle_{c d}$ can be written as:

$$
\begin{aligned}
& \left|\Psi^{\prime}\right\rangle_{a b} \otimes\left|\Psi^{\prime}\right\rangle_{c d}=\left(\alpha a^{\dagger}+\beta e^{i \theta_{A B}} b^{\dagger}\right) \otimes\left(\alpha c^{\dagger}+\beta e^{i \theta_{C D}} d^{\dagger}\right)|0\rangle \\
& =\left(\alpha^{2} a^{\dagger} c^{\dagger}+\beta^{2} e^{i\left(\theta_{A B}+\theta_{C D}\right)} b^{\dagger} d^{\dagger}\right)|0\rangle+\left(\alpha \beta e^{i \theta_{A B}} b^{\dagger} c^{\dagger}+\alpha \beta e^{i \theta_{C D}} a^{\dagger} d^{\dagger}\right)|0\rangle
\end{aligned}
$$

Here, we let $\left|\Psi^{\prime}\right\rangle_{c d}$ have the same form as $\left|\Psi^{\prime}\right\rangle_{a b}$, i.e., $\left|\Psi^{\prime}\right\rangle_{c d}=\left(\alpha c^{\dagger}+\beta e^{i \theta_{C D}} d^{\dagger}\right)|0\rangle$. The BS will make $b^{\dagger}|0\rangle \rightarrow \frac{1}{\sqrt{2}}\left(D_{1}^{\dagger}+D_{2}^{\dagger}\right)|0\rangle$ and $c^{\dagger}|0\rangle \rightarrow \frac{1}{\sqrt{2}}\left(D_{1}^{\dagger}-D_{2}^{\dagger}\right)|0\rangle$. After BS, from Equation (49), we can find that if one of the detectors clicks one photon, we will get:

$$
\left|\Psi^{\prime \prime}\right\rangle_{a d}=\left(\alpha^{2} a^{\dagger} \pm \beta^{2} d^{\dagger} e^{i\left(\theta_{A B}+\theta_{C D}\right)}\right)|0\rangle \text {. }
$$

The "+" or "-" depends on the click of the detector, $D_{1}$ or $D_{2}$.

Figure 10. The setup of entanglement connection in the Duan-Lukin-Cirac-Zoller (DLCZ) protocol [16]. BS is a 50:50 beam splitter [55]. After this swapping, if one of the detectors registers exactly one photon, the entanglement between $M_{A}$ and $M_{D}$ can be set up.

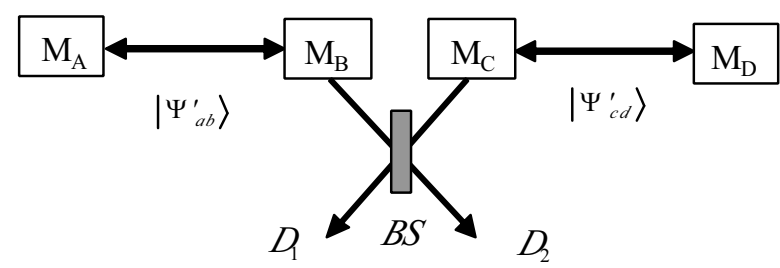

From Equation (50), the entanglement will be degraded after the entanglement connection, as shown in Figure 10. If we consider the case that we perform the entanglement swapping for $n$ times to connect the entanglement between the remote locations, $A$ and $K$, we will get:

$$
\left|\Psi^{n+1}\right\rangle_{a k}=\left(\alpha^{n+1} a^{\dagger} \pm \beta^{n+1} k^{\dagger} e^{i \theta_{A K}}\right)|0\rangle
$$

For $\alpha \neq \beta$, the entanglement decreases rapidly, so that we cannot establish a perfect long-distance entanglement channel for quantum communication.

Fortunately, entanglement concentration provides us a good way to convert the nonmaximally entangled state, like Equation (51), to a maximally entangled state. The principle of our single-photon ECP is shown in Figure 11 [55]. Alice and Bob want to share the maximally entangled state, $|\Psi\rangle_{a b}=\frac{1}{\sqrt{2}}\left(a^{\dagger}+b^{\dagger}\right)|0\rangle$. But the initial state shared by Alice and Bob is:

$$
\begin{aligned}
|\Psi\rangle_{a_{1} b_{1}} & =\left(\alpha a_{1}^{\dagger}+\beta e^{i \theta_{A_{1} B_{1}}} b_{1}^{\dagger}\right)|0\rangle \\
|\Psi\rangle_{a_{2} b_{2}} & =\left(\alpha a_{2}^{\dagger}+\beta e^{i \theta_{A_{2} B_{2}}} b_{2}^{\dagger}\right)|0\rangle
\end{aligned}
$$


Figure 11. The principle of the single-photon ECP [55]. A BS is located in the middle of $\mathrm{A}$ and $\mathrm{B}$, and it is used to couple the two modes, $a_{2}$ and $b_{2}$. A homodyne detector is used to distinguish the photon number. After the detection of $D_{1}$ and $D_{2}$, the two parties can get some maximally entangled states with the probability $2|\alpha \beta|^{2}$.

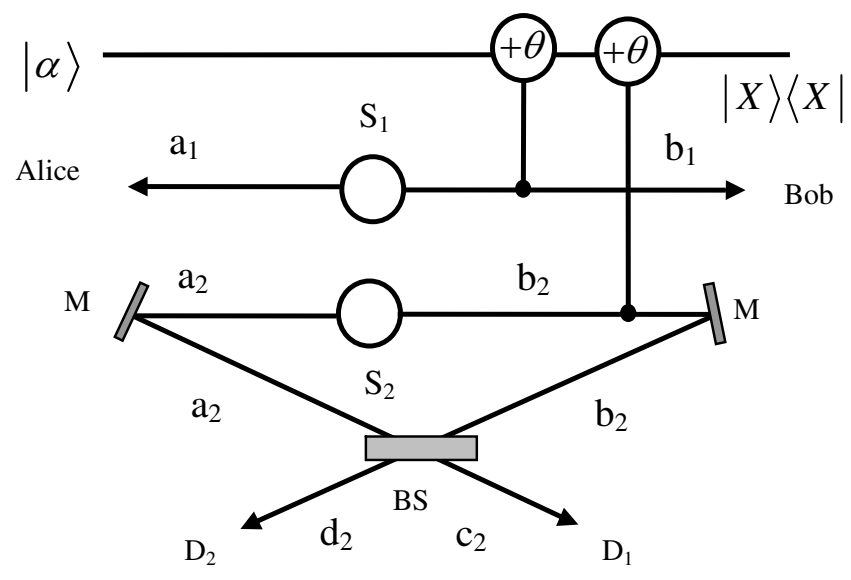

Here, $a_{1} b_{1}$ and $a_{2} b_{2}$ are the different spatial modes shown in Figure 11. In this protocol, we neglect the path length fluctuations of two channels, $a_{1} b_{1}$ and $a_{2} b_{2}$, and suppose that the two sources emit the entangled state simultaneously. In this way, the relative phase, $\theta_{A_{1} B_{1}}$ and $\theta_{A_{2} B_{2}}$, can sever as the same. The combination of the two partially entangled state can be written as:

$$
|\Phi\rangle=|\Psi\rangle_{a_{1} b_{1}} \otimes|\Psi\rangle_{a_{2} b_{2}}=\left(\alpha^{2} a_{1}^{\dagger} a_{2}^{\dagger}+\alpha \beta e^{i \theta_{A B}} a_{1}^{\dagger} b_{2}^{\dagger}+\alpha \beta e^{i \theta_{A B}} a_{2}^{\dagger} b_{1}^{\dagger}+\beta^{2} e^{2 i \theta_{A B}} b_{1}^{\dagger} b_{2}^{\dagger}\right)|0\rangle
$$

$a_{1}^{\dagger} a_{2}^{\dagger}$ and $b_{1}^{\dagger} b_{2}^{\dagger}$ represent that the two photons both belong to Alice and Bob, respectively. From the above equation, it is obvious that $a_{1}^{\dagger} b_{2}^{\dagger}$ and $a_{2}^{\dagger} b_{1}^{\dagger}$ have the same coefficient, $\alpha \beta e^{i \theta_{A B}}$, and the other two terms have different coefficients. Therefore, if Bob makes a homodyne measurement on the coherent state, he will get three different results. The first one is the phase shift, $\theta$, which corresponds to the items $a_{1}^{\dagger} b_{2}^{\dagger}$ and $a_{2}^{\dagger} b_{1}^{\dagger}$. The second result is $2 \theta$, which corresponds to the item $b_{1}^{\dagger} b_{2}^{\dagger}$, and the last one is 0 , which corresponds to the item $a_{1}^{\dagger} b_{2}^{\dagger}$. Bob will require Alice to keep her result if the phase shift is $\theta$. In this way, if we omit the global phase shift factor, $e^{i \theta_{A B}}$, then the original state will collapse to:

$$
|\Phi\rangle^{\prime}=\frac{1}{\sqrt{2}}\left(a_{1}^{\dagger} b_{2}^{\dagger}+a_{2}^{\dagger} b_{1}^{\dagger}\right)|0\rangle
$$

The probability that Alice and Bob get the state, $|\Phi\rangle^{\prime}$, is $2|\alpha \beta|^{2}$. The modes, $a_{2}$ and $b_{2}$, are reflected and coupled by a 50:50 BS, which will make:

$$
\begin{aligned}
& a_{2}^{\dagger}|0\rangle \rightarrow \frac{1}{\sqrt{2}}\left(c_{2}^{\dagger}-d_{2}^{\dagger}\right)|0\rangle \\
& b_{2}^{\dagger}|0\rangle \rightarrow \frac{1}{\sqrt{2}}\left(c_{2}^{\dagger}+d_{2}^{\dagger}\right)|0\rangle
\end{aligned}
$$

After the BS, Equation (55) can evolve to:

$$
|\Phi\rangle^{\prime} \rightarrow \frac{1}{2}\left[a_{1}^{\dagger}\left(c_{2}^{\dagger}+d_{2}^{\dagger}\right)+b_{1}^{\dagger}\left(c_{2}^{\dagger}-d_{2}^{\dagger}\right)\right]|0\rangle=\frac{1}{2}\left[\left(a_{1}^{\dagger}+b_{1}^{\dagger}\right) c_{2}^{\dagger}+\left(a_{1}^{\dagger}-b_{1}^{\dagger}\right) d_{2}^{\dagger}\right]|0\rangle
$$


One can see that if the detector, $D_{1}$, fires, the state of the remaining quantum system will be left to:

$$
\left|\Phi_{1}\right\rangle^{\prime}=\frac{1}{\sqrt{2}}\left(a_{1}^{\dagger}+b_{1}^{\dagger}\right)|0\rangle
$$

otherwise, the detector, $D_{2}$, fires, and the quantum system will collapse to:

$$
\left|\Phi_{2}\right\rangle^{\prime}=\frac{1}{\sqrt{2}}\left(a_{1}^{\dagger}-b_{1}^{\dagger}\right)|0\rangle
$$

Equations (59) and (60) are both maximally single-photon entangled states. There is a phase difference between them, and one can perform a phase-flipping operation with the help of the wave plate to convert Equation (60) to Equation (59).

So far, we have explained the basic principle of this concentration protocol. Alice and Bob pick up the case that the phase shift is $\theta$ with the probability of $2|\alpha \beta|^{2}$. In fact, similar to the above section, the yield can also be increased if a suitable cross-Kerr material can be provided, or the interaction time can be controlled accurately. This time, Alice and Bob will get the phase shift, $\theta=\pi$, when one photon is detected, and one cannot distinguish the phase shift, 0 and $2 \pi$. In this case, Equation (54) will collapse to:

$$
|\Phi\rangle^{\prime \prime}=\left(\alpha^{2} a_{1}^{\dagger} a_{2}^{\dagger}+\beta^{2} e^{2 i \theta_{A B}} b_{1}^{\dagger} b_{2}^{\dagger}\right)|0\rangle
$$

In the next step, one can also get the maximal one with single-photon entanglement concentration. In detail, after coupled by BS, Equation (54) collapses to:

$$
\begin{aligned}
|\Phi\rangle^{\prime \prime} & =\left[\frac{\alpha^{2}}{\sqrt{2}} a_{1}^{\dagger}\left(c_{2}^{\dagger}-d_{2}^{\dagger}\right)+\frac{\beta^{2} e^{2 i \theta_{A B}}}{\sqrt{2}} b_{1}^{\dagger}\left(c_{2}^{\dagger}+d_{2}^{\dagger}\right)\right]|0\rangle \\
& =\left[\left(\frac{\alpha^{2}}{\sqrt{2}} a_{1}^{\dagger}-\frac{\beta^{2} e^{2 i \theta_{A B}}}{\sqrt{2}} b_{1}^{\dagger}\right) c_{2}^{\dagger}+\left(\frac{\alpha^{2}}{\sqrt{2}} a_{1}^{\dagger}+\frac{\beta^{2} e^{2 i \theta_{A B}}}{\sqrt{2}} b_{1}^{\dagger}\right) d_{2}^{\dagger}\right]|0\rangle
\end{aligned}
$$

If the detector, $D_{1}$, fires, the state in Equation (62) will be transformed to:

$$
\left|\Phi_{1}\right\rangle^{\prime \prime}=\left(\alpha^{2} a_{1}^{\dagger}-\beta^{2} e^{2 i \theta_{A B}} b_{1}^{\dagger}\right)|0\rangle
$$

If the detector, $D_{2}$, fires, it will be transformed to:

$$
\left|\Phi_{2}\right\rangle^{\prime \prime}=\left(\alpha^{2} a_{1}^{\dagger}+\beta^{2} e^{2 i \theta_{A B}} b_{1}^{\dagger}\right)|0\rangle
$$

Compared with Equation (48), Equation (64) has the same form as Equation (48) and can be reconcentrated. In the next concentration step, we choose two copies of these partially entangled states, and the whole system becomes:

$$
|\Phi\rangle^{\prime \prime \prime}=\left|\Phi_{2}\right\rangle_{a_{1} b_{1}}^{\prime \prime} \otimes\left|\Phi_{2}\right\rangle_{a_{2} b_{2}}^{\prime \prime}=\left(\alpha^{4} a_{1}^{\dagger} a_{2}^{\dagger}+\alpha^{2} \beta^{2} e^{i 2 \theta_{A B}} a_{1}^{\dagger} b_{2}^{\dagger}+\alpha^{2} \beta^{2} e^{i 2 \theta_{A B}} a_{2}^{\dagger} b_{1}^{\dagger}+\beta^{4} e^{i 4 \theta_{A B}} b_{1}^{\dagger} b_{2}^{\dagger}\right)|0\rangle
$$

Alice and Bob pick up the case that the phase shift is also $\theta$ with the success probability of $2\left|\alpha^{2} \beta^{2}\right|^{2}$, and they keep the other terms for the next iteration. Equation (63) can also be manipulated with the same step as that discussed above. In this way, this ECP can also be repeated to obtain a higher success probability. 


\section{ECPs for W States}

In the three-particle system, there are two classes of tripartite-entangled states, which cannot be converted into each other by stochastic local operations and classical communication. They are the GHZ state and the $\mathrm{W}$ state. The GHZ state can be written as $|G H Z\rangle=\frac{1}{\sqrt{2}}(|000\rangle+|111\rangle)$, and the $\mathrm{W}$ state can be written as $|W\rangle=\frac{1}{\sqrt{3}}(|001\rangle+|010\rangle+|100\rangle)$. The concentration for the GHZ state can be easily extended from the ECPs for the Bell state in Section 2. However, the ECPs described in Section 2 cannot deal with the W state. Certainly, there are several ECPs for the W state, such as the ECP with joint unitary transformation proposed by Cao and Yang [57], the ECP based on the Bell-state measurement [58]. Both joint unitary transformation and Bell-state measurement are not easy to realize in the current condition. There are ECPs for some special types of W states, such as $\alpha|H H V\rangle+\beta(|H V H\rangle+|V H H\rangle)$, and the asymmetric W states [59]

In this section, we will describe the ECP for concentrating arbitrary $\mathrm{W}$ stat, $\alpha|V H H\rangle+\beta|H V H\rangle+$ $\gamma|H H V\rangle$, to a standard maximally entangled $\mathrm{W}$ state, $\frac{1}{\sqrt{3}}(|V H H\rangle+|H V H\rangle+|H H V\rangle)[60]$. We also extend this idea to concentrate the single-photon multi-mode $\mathrm{W}$ state [61]. We will show that both linear optics and nonlinear optics can achieve the task. Interestingly, different from the other ECPs, these ECPs only require some single photons $[60,61]$.

\subsection{Two-Step Entanglement Concentration for Arbitrary W States}

We will describe two ECPs for concentrating the arbitrary W state, $\alpha|V H H\rangle+\beta|H V H\rangle+\gamma|H H V\rangle$. The first one uses linear optics, and the second one uses the cross-Kerr nonlinearity. From Figure 12, suppose a pair of partially entangled W states, $|\Phi\rangle_{a 1 b 1 c 1}$, is sent to Alice, Bob and Charlie [60]. The photon pair is initially in the following polarization partially entangled state:

$$
|\Phi\rangle_{a 1 b 1 c 1}=\alpha|V\rangle_{a 1}|H\rangle_{b 1}|H\rangle_{c 1}+\beta|H\rangle_{a 1}|V\rangle_{b 1}|H\rangle_{c 1}+\gamma|H\rangle_{a 1}|H\rangle_{b 1}|V\rangle_{c 1}
$$

We let $\alpha, \beta$ and $\gamma$ be real for simplicity, with $\alpha^{2}+\beta^{2}+\gamma^{2}=1$. The source, $S_{2}$, emits a single photon of the form:

$$
|\Phi\rangle_{a 2}=\frac{\alpha}{\sqrt{\alpha^{2}+\beta^{2}}}|H\rangle_{a 2}+\frac{\beta}{\sqrt{\alpha^{2}+\beta^{2}}}|V\rangle_{a 2}
$$

In this way, the whole system can be written as:

$$
\begin{aligned}
|\Psi\rangle & =|\Phi\rangle_{a 1 b 1 c 1} \otimes|\Phi\rangle_{a 2}=\left(\alpha|V\rangle_{a 1}|H\rangle_{b 1}|H\rangle_{c 1}+\beta|H\rangle_{a 1}|V\rangle_{b 1}|H\rangle_{c 1}+\gamma|H\rangle_{a 1}|H\rangle_{b 1}|V\rangle_{c 1}\right) \\
& \otimes\left(\frac{\alpha}{\sqrt{\alpha^{2}+\beta^{2}}}|H\rangle_{a 2}+\frac{\beta}{\sqrt{\alpha^{2}+\beta^{2}}}|V\rangle_{a 2}\right) \\
& =\frac{\alpha^{2}}{\sqrt{\alpha^{2}+\beta^{2}}}|V\rangle_{a 1}|H\rangle_{a 2}|H\rangle_{b 1}|H\rangle_{c 1}+\frac{\beta^{2}}{\sqrt{\alpha^{2}+\beta^{2}}}|H\rangle_{a 1}|V\rangle_{a 2}|V\rangle_{b 1}|H\rangle_{c 1} \\
& +\frac{\alpha \gamma}{\sqrt{\alpha^{2}+\beta^{2}}}|H\rangle_{a 1}|H\rangle_{a 2}|H\rangle_{b 1}|V\rangle_{c 1}+\frac{\beta \gamma}{\sqrt{\alpha^{2}+\beta^{2}}}|H\rangle_{a 1}|V\rangle_{a 2}|H\rangle_{b 1}|V\rangle_{c 1} \\
& +\frac{\alpha \beta}{\sqrt{\alpha^{2}+\beta^{2}}}|V\rangle_{a 1}|V\rangle_{a 2}|H\rangle_{b 1}|H\rangle_{c 1}+\frac{\alpha \beta}{\sqrt{\alpha^{2}+\beta^{2}}}|H\rangle_{a 1}|H\rangle_{a 2}|V\rangle_{b 1}|H\rangle_{c 1}
\end{aligned}
$$


If they choose the cases that the spatial modes, $d 1, d 2, b 1$ and $c 1$, all contain exactly one photon, the initial state will become:

$$
\begin{aligned}
|\Psi\rangle^{\prime} & =\frac{\alpha \gamma}{\sqrt{\alpha^{2}+\beta^{2}}}|H\rangle_{d 1}|H\rangle_{d 2}|H\rangle_{b 1}|V\rangle_{c 1}+\frac{\alpha \beta}{\sqrt{\alpha^{2}+\beta^{2}}}|V\rangle_{d 1}|V\rangle_{d 2}|H\rangle_{b 1}|H\rangle_{c 1} \\
& +\frac{\alpha \beta}{\sqrt{\alpha^{2}+\beta^{2}}}|H\rangle_{d 1}|H\rangle_{d 2}|V\rangle_{b 1}|H\rangle_{c 1}
\end{aligned}
$$

The probability is:

$$
P^{1}=\frac{\alpha^{2}\left(\gamma^{2}+2 \beta^{2}\right)}{\alpha^{2}+\beta^{2}}
$$

The superscript " 1 " means the first concentration step.

Figure 12. Schematic drawing of the first ECP with linear optics [60]. $S_{1}$ is the partial entanglement source and $S_{2}$ and $S_{3}$ are the single photon sources. $\mathrm{HWP}_{90}$ and $\mathrm{HWP}_{45}$ can rotate the polarization of the state by $90^{\circ}$ and $45^{\circ}$, respectively.

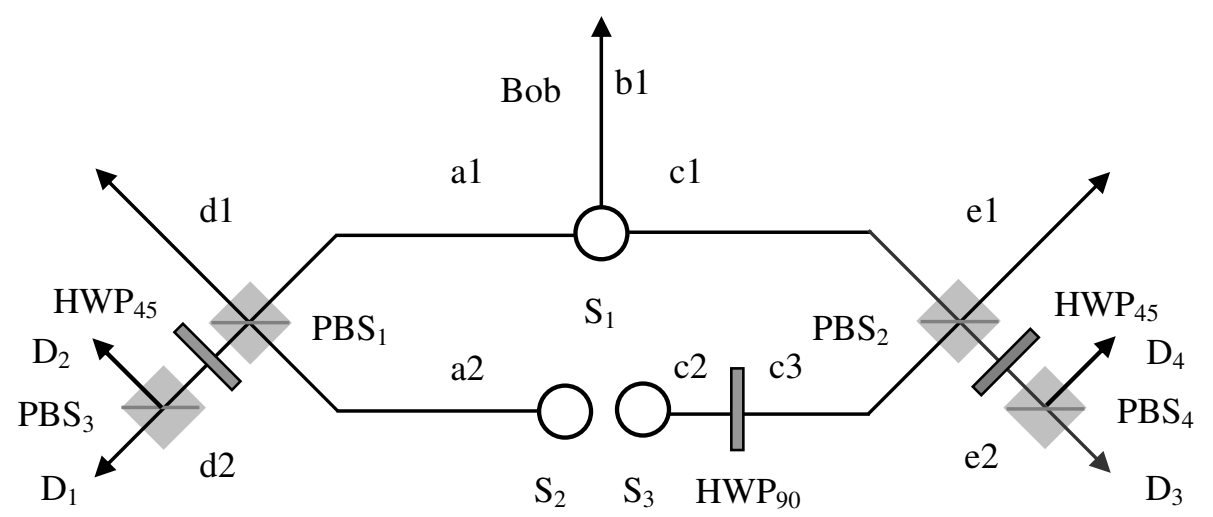

Alice

Charlie

Equation (69) can be rewritten as:

$$
\begin{aligned}
|\Psi\rangle^{\prime} & =\frac{\gamma}{\sqrt{\gamma^{2}+2 \beta^{2}}}|H\rangle_{d 1}|H\rangle_{d 2}|H\rangle_{b 1}|V\rangle_{c 1}+\frac{\beta}{\sqrt{\gamma^{2}+2 \beta^{2}}}|V\rangle_{d 1}|V\rangle_{d 2}|H\rangle_{b 1}|H\rangle_{c 1} \\
& +\frac{\beta}{\sqrt{\gamma^{2}+2 \beta^{2}}}|H\rangle_{d 1}|H\rangle_{d 2}|V\rangle_{b 1}|H\rangle_{c 1}
\end{aligned}
$$

From Equation (71), the initial coefficient, $\alpha$, is eliminated. Then, Alice rotates the photons in spatial mode, $d 2$, with $\lambda / 4$-wave plate $\mathrm{HWP}_{45}$ to perform a Hadamard operation and measures it with D1 and D2. If the photon in spatial mode, $d 2$, is $|H\rangle_{d 2}$, which makes detector $\mathrm{D}_{1}$ fire, the original state will be left in the state:

$$
\begin{aligned}
\left|\Phi_{1}\right\rangle_{d 1 b 1 c 1} & =\frac{\gamma}{\sqrt{\gamma^{2}+2 \beta^{2}}}|H\rangle_{d 1}|H\rangle_{b 1}|V\rangle_{c 1}+\frac{\beta}{\sqrt{\gamma^{2}+2 \beta^{2}}}|V\rangle_{d 1}|H\rangle_{b 1}|H\rangle_{c 1} \\
& +\frac{\beta}{\sqrt{\gamma^{2}+2 \beta^{2}}}|H\rangle_{d 1}|V\rangle_{b 1}|H\rangle_{c 1}
\end{aligned}
$$


Otherwise, if $\mathrm{D}_{2}$ fires, the original state will be left in the state:

$$
\begin{aligned}
\left|\Phi_{2}\right\rangle_{d 1 b 1 c 1} & =\frac{\gamma}{\sqrt{\gamma^{2}+2 \beta^{2}}}|H\rangle_{d 1}|H\rangle_{b 1}|V\rangle_{c 1}-\frac{\beta}{\sqrt{\gamma^{2}+2 \beta^{2}}}|V\rangle_{d 1}|H\rangle_{b 1}|H\rangle_{c 1} \\
& +\frac{\beta}{\sqrt{\gamma^{2}+2 \beta^{2}}}|H\rangle_{d 1}|V\rangle_{b 1}|H\rangle_{c 1}
\end{aligned}
$$

In order to get $\left|\Phi_{1}\right\rangle_{d 1 b 1 c 1}$, one of the parties, say, Alice, Bob or Charlie, should perform a local operation of phase rotation on her or his photon.

The second concentration step is analogous with the first one performed by Charlie. The source, $S_{3}$, emits a single photon of the form:

$$
|\Phi\rangle_{c 2}=\frac{\beta}{\sqrt{\gamma^{2}+\beta^{2}}}|H\rangle_{c 2}+\frac{\gamma}{\sqrt{\gamma^{2}+\beta^{2}}}|V\rangle_{c 2}
$$

Charlie first rotates the photon by $90^{\circ}$ in the spatial mode, $c 2$, with $\mathrm{HWP}_{90}$. The $|\Phi\rangle_{c 2}$ can be written as:

$$
|\Phi\rangle_{c 3}=\frac{\beta}{\sqrt{\gamma^{2}+\beta^{2}}}|V\rangle_{c 3}+\frac{\gamma}{\sqrt{\gamma^{2}+\beta^{2}}}|H\rangle_{c 3}
$$

Certainly, one can also first perform the single-photon of the form of Equation (75). We prepare the state, $|\Phi\rangle_{c 2}$, to have the same form of $|\Phi\rangle_{a 2}$ and make the whole protocol have the same form. The combination of the four-photon state can be written as:

$$
\begin{aligned}
& \left|\Phi_{1}\right\rangle_{d 1 b 1 c 1} \otimes|\Phi\rangle_{c 3}=\frac{\beta \gamma}{\sqrt{\gamma^{2}+2 \beta^{2}} \sqrt{\gamma^{2}+\beta^{2}}}|H\rangle_{d 1}|H\rangle_{b 1}|V\rangle_{c 1}|V\rangle_{c 3} \\
+ & \frac{\gamma^{2}}{\sqrt{\gamma^{2}+2 \beta^{2}} \sqrt{\gamma^{2}+\beta^{2}}}|H\rangle_{d 1}|H\rangle_{b 1}|V\rangle_{c 1}|H\rangle_{c 3}+\frac{\beta^{2}}{\sqrt{\gamma^{2}+2 \beta^{2}} \sqrt{\gamma^{2}+\beta^{2}}}|V\rangle_{d 1}|H\rangle_{b 1}|H\rangle_{c 1}|V\rangle_{c 3} \\
+ & \frac{\beta \gamma}{\sqrt{\gamma^{2}+2 \beta^{2}} \sqrt{\gamma^{2}+\beta^{2}}}|V\rangle_{d 1}|H\rangle_{b 1}|H\rangle_{c 1}|H\rangle_{c 3}+\frac{\beta^{2}}{\sqrt{\gamma^{2}+2 \beta^{2}} \sqrt{\gamma^{2}+\beta^{2}}}|H\rangle_{d 1}|V\rangle_{b 1}|H\rangle_{c 1}|V\rangle_{c 3} \\
+ & \frac{\beta \gamma}{\sqrt{\gamma^{2}+2 \beta^{2}} \sqrt{\gamma^{2}+\beta^{2}}}|H\rangle_{d 1}|V\rangle_{b 1}|H\rangle_{c 1}|H\rangle_{c 3}
\end{aligned}
$$

With the same principle, Charlie chooses the cases that two output modes of $\mathrm{PBS}_{2}$ both exactly contain only one photon, and the Equation (76) becomes:

$$
\left|\Psi^{\prime \prime \prime}\right\rangle=\frac{1}{\sqrt{3}}\left(|H\rangle_{d 1}|H\rangle_{b 1}|V\rangle_{e 1}|V\rangle_{e 2}+|V\rangle_{d 1}|H\rangle_{b 1}|H\rangle_{e 1}|H\rangle_{e 2}+|H\rangle_{d 1}|V\rangle_{b 1}|H\rangle_{e 1}|H\rangle_{e 2}\right)
$$

with a success probability of:

$$
P^{2}=\frac{3 \beta^{2} \gamma^{2}}{\left(\gamma^{2}+\beta^{2}\right)\left(\gamma^{2}+2 \beta^{2}\right)}
$$

Here, the superscript " 2 " means the second concentration step. Finally, Charlie rotates his photon in the mode, $e 2$, by $45^{\circ}$ and measures the photon in spatial mode, $e 2$. If $\mathrm{D}_{3}$ fires, they will get:

$$
\left|\Phi_{1}\right\rangle_{d 1 b 1 e 1}=\frac{1}{\sqrt{3}}\left(|H\rangle_{d 1}|H\rangle_{b 1}|V\rangle_{e 1}+|V\rangle_{d 1}|H\rangle_{b 1}|H\rangle_{e 1}+|H\rangle_{d 1}|V\rangle_{b 1}|H\rangle_{e 1}\right)
$$


If $\mathrm{D}_{4}$ fires, they will get:

$$
\left|\Phi_{2}\right\rangle_{d 1 b 1 e 1}=\frac{1}{\sqrt{3}}\left(-|H\rangle_{d 1}|H\rangle_{b 1}|V\rangle_{e 1}+|V\rangle_{d 1}|H\rangle_{b 1}|H\rangle_{e 1}+|H\rangle_{d 1}|V\rangle_{b 1}|H\rangle_{e 1}\right)
$$

Both Equations (79) and (80) are the maximally entangled W states. In order to get $\left|\Phi_{1}\right\rangle_{d 1 b 1 e 1}$, one of three parties, says Alice, Bob or Charlie, should perform a local operation of phase rotation on her or his photon.

The total success probability, $P_{s}$, for obtaining a maximally entangled $\mathrm{W}$ state is:

$$
P_{s}=P^{1} P^{2}=\frac{\alpha^{2}\left(\gamma^{2}+2 \beta^{2}\right)}{\alpha^{2}+\beta^{2}} \frac{3 \beta^{2} \gamma^{2}}{\left(\gamma^{2}+\beta^{2}\right)\left(\gamma^{2}+2 \beta^{2}\right)}=\frac{3 \alpha^{2} \beta^{2} \gamma^{2}}{\left(\alpha^{2}+\beta^{2}\right)\left(\gamma^{2}+\beta^{2}\right)}
$$

In the above description, the total ECP is divided into two steps. The first one is performed by Alice, and the second one is performed by Charlie. In the practical operation, they should perform the ECP simultaneously, because of the post-selection principle. On the other hand, they should resort to the sophisticated single-photon detectors to check the photon number. So, it is not an optimal ECP. In the second ECP, we use the QND to substitute the PBS to redescribe this ECP. The QND is described in Figure 3. The principle of the second ECP is shown in Figure 13. The first step is also performed by Alice. The four photons combined with the coherent state can be written as:

$$
\begin{aligned}
|\Psi\rangle|\alpha\rangle & =|\Phi\rangle_{a 1 b 1 c 1} \otimes|\Phi\rangle_{a 2}|\alpha\rangle=\left(\alpha|V\rangle_{a 1}|H\rangle_{b 1}|H\rangle_{c 1}+\beta|H\rangle_{a 1}|V\rangle_{b 1}|H\rangle_{c 1}+\gamma|H\rangle_{a 1}|H\rangle_{b 1}|V\rangle_{c 1}\right) \\
& \otimes\left(\frac{\alpha}{\sqrt{\alpha^{2}+\beta^{2}}}|H\rangle_{a 2}+\frac{\beta}{\sqrt{\alpha^{2}+\beta^{2}}}|V\rangle_{a 2}\right)|\alpha\rangle \\
& \rightarrow \frac{\alpha^{2}}{\sqrt{\alpha^{2}+\beta^{2}}}|V\rangle_{a 1}|H\rangle_{a 2}|H\rangle_{b 1}|H\rangle_{c 1}|\alpha\rangle+\frac{\beta^{2}}{\sqrt{\alpha^{2}+\beta^{2}}}|H\rangle_{a 1}|V\rangle_{a 2}|V\rangle_{b 1}|H\rangle_{c 1}\left|\alpha e^{i 2 \theta}\right\rangle \\
& +\frac{\alpha \gamma}{\sqrt{\alpha^{2}+\beta^{2}}}|H\rangle_{a 1}|H\rangle_{a 2}|H\rangle_{b 1}|V\rangle_{c 1}\left|\alpha e^{i \theta}\right\rangle+\frac{\beta \gamma}{\sqrt{\alpha^{2}+\beta^{2}}}|H\rangle_{a 1}|V\rangle_{a 2}|H\rangle_{b 1}|V\rangle_{c 1}\left|\alpha e^{i 2 \theta}\right\rangle \\
& +\frac{\alpha \beta}{\sqrt{\alpha^{2}+\beta^{2}}}|V\rangle_{a 1}|V\rangle_{a 2}|H\rangle_{b 1}|H\rangle_{c 1}\left|\alpha e^{i \theta}\right\rangle+\frac{\alpha \beta}{\sqrt{\alpha^{2}+\beta^{2}}}|H\rangle_{a 1}|H\rangle_{a 2}|V\rangle_{b 1}|H\rangle_{c 1}\left|\alpha e^{i \theta}\right\rangle
\end{aligned}
$$

After the photons pass through the QND1, if Alice picks up the phase shift, $\theta$, the remaining state essentially will be Equation (69). Certainly, the similar step is performed by Charlie. The $\left|\Phi_{1}\right\rangle_{d 1 b 1 c 1}$ and $|\Phi\rangle_{c 3}$ combined with the coherent state evolves as:

$$
\begin{aligned}
& |\Phi\rangle_{c 3} \otimes\left|\Phi_{1}\right\rangle_{d 1 b 1 c 1}|\alpha\rangle \rightarrow \frac{\beta \gamma}{\sqrt{\gamma^{2}+2 \beta^{2}} \sqrt{\gamma^{2}+\beta^{2}}}|H\rangle_{d 1}|H\rangle_{b 1}|V\rangle_{c 1}|V\rangle_{e 2}\left|\alpha e^{i \theta}\right\rangle \\
+ & \frac{\gamma^{2}}{\sqrt{\gamma^{2}+2 \beta^{2}} \sqrt{\gamma^{2}+\beta^{2}}}|H\rangle_{d 1}|H\rangle_{b 1}|V\rangle_{c 1}|H\rangle_{e 2}|\alpha\rangle \\
+ & \frac{\beta^{2}}{\sqrt{\gamma^{2}+2 \beta^{2}} \sqrt{\gamma^{2}+\beta^{2}}}|V\rangle_{d 1}|H\rangle_{b 1}|H\rangle_{c 1}|V\rangle_{e 2}\left|\alpha e^{i 2 \theta}\right\rangle \\
+ & \frac{\beta \gamma}{\sqrt{\gamma^{2}+2 \beta^{2}} \sqrt{\gamma^{2}+\beta^{2}}}|V\rangle_{d 1}|H\rangle_{b 1}|H\rangle_{c 1}|H\rangle_{e 2}\left|\alpha e^{i \theta}\right\rangle \\
+ & \frac{\beta^{2}}{\sqrt{\gamma^{2}+2 \beta^{2}} \sqrt{\gamma^{2}+\beta^{2}}}|H\rangle_{d 1}|V\rangle_{b 1}|H\rangle_{c 1}|V\rangle_{e 2}\left|\alpha e^{i 2 \theta}\right\rangle \\
+ & \frac{\beta \gamma}{\sqrt{\gamma^{2}+2 \beta^{2}} \sqrt{\gamma^{2}+\beta^{2}}}|H\rangle_{d 1}|V\rangle_{b 1}|H\rangle_{c 1}|H\rangle_{e 2}\left|\alpha e^{i \theta}\right\rangle
\end{aligned}
$$


From Equation (83), if Charlie also picks up the $\theta$ phase shift, the remaining state essentially will be the four-photon maximally entangled $\mathrm{W}$ state, which can be used to obtain the three-photon maximally entangled W state by measuring one of the photons. From the above description, both Alice and Charlie pick up the $\theta$ shift of the coherent state. If a suitable cross-Kerr nonlinearity can be provided, and the interaction time, $t$, can be well controlled, which leads to $\theta=\pi$. In this way, phase shift, $2 \theta=2 \pi$ and 0 , will not be distinguished. Therefore, the discarded items in each step are the nonmaximally entangled $\mathrm{W}$ states and can be reconcentrated in the next round. For example, if the phase shift in Charlie's location is not $\theta$, yet, then the Equation (83) will become:

$$
\begin{gathered}
\left|\Psi_{2}\right\rangle_{d 1 b 1 c 1 e 2}^{\prime}=\frac{\gamma^{2}}{\sqrt{\gamma^{2}+2 \beta^{2}} \sqrt{\gamma^{2}+\beta^{2}}}|H\rangle_{d 1}|H\rangle_{b 1}|V\rangle_{c 1}|H\rangle_{e 2} \\
+\frac{\beta^{2}}{\sqrt{\gamma^{2}+2 \beta^{2}} \sqrt{\gamma^{2}+\beta^{2}}}|V\rangle_{d 1}|H\rangle_{b 1}|H\rangle_{c 1}|V\rangle_{e 2}+\frac{\beta^{2}}{\sqrt{\gamma^{2}+2 \beta^{2}} \sqrt{\gamma^{2}+\beta^{2}}}|H\rangle_{d 1}|V\rangle_{b 1}|H\rangle_{c 1}|V\rangle_{e 2}
\end{gathered}
$$

Figure 13. Schematic drawing of the second ECP with the cross-Kerr nonlinearity [60]. Compared with Figure 12, we use two QNDs described in Figure 3 to substitute the two PBSs. It can reach a higher success probability than the first ECP.

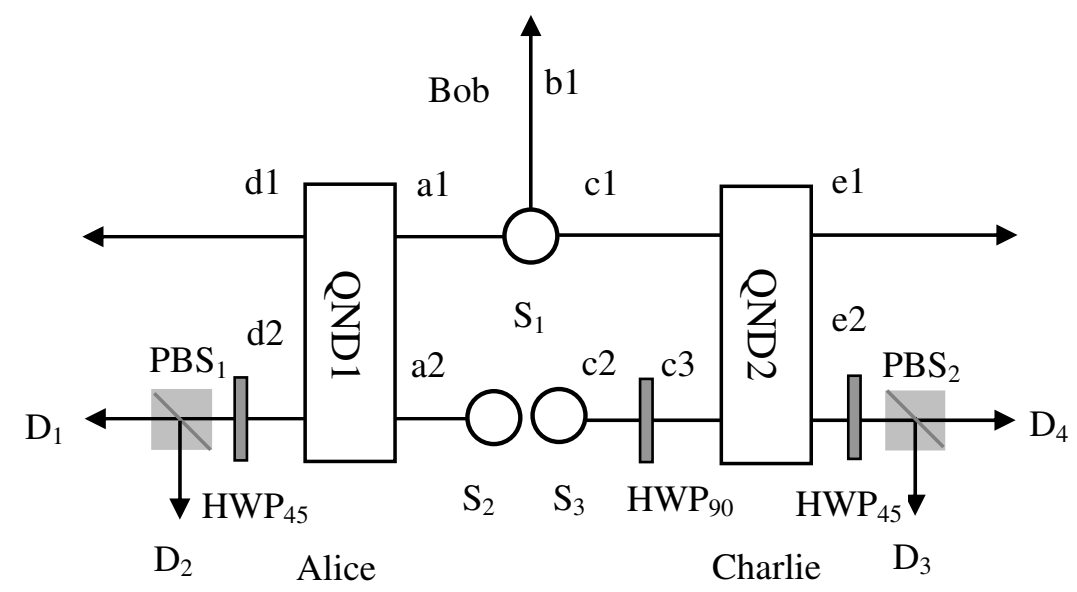

By measuring the photon in mode, $e 2$, after rotating it by $45^{\circ}$, it becomes:

$$
\left|\Psi_{2}^{ \pm}\right\rangle_{d 1 b 1 c 1}^{\prime}=\gamma^{\prime \prime}|H\rangle_{d 1}|H\rangle_{b 1}|V\rangle_{c 1} \pm \beta^{\prime \prime}|V\rangle_{d 1}|H\rangle_{b 1}|H\rangle_{c 1} \pm \beta^{\prime \prime}|H\rangle_{d 1}|V\rangle_{b 1}|H\rangle_{c 1}
$$

Here we let:

$$
\begin{aligned}
& \gamma^{\prime \prime}=\frac{\gamma^{2}}{\sqrt{\gamma^{4}+2 \beta^{4}}} \\
& \beta^{\prime \prime}=\frac{\beta^{2}}{\sqrt{\gamma^{4}+2 \beta^{4}}}
\end{aligned}
$$

"+" or "-" also depend on the measurement result. If $\mathrm{D}_{3}$ fires, it is "+", otherwise, it is "-_". In this way, the remaining state in Equation (85) can be reconcentrated in the next round. In detail, Charlie chooses another single photon of the form:

$$
\left|\Phi^{\prime}\right\rangle_{c 2}=\frac{\beta^{2}}{\sqrt{\gamma^{4}+\beta^{4}}}|H\rangle_{c 2}+\frac{\gamma^{2}}{\sqrt{\gamma^{4}+\beta^{4}}}|V\rangle_{c 2}
$$


After rotating this photon by $90^{\circ}$, it becomes:

$$
\left|\Phi^{\prime}\right\rangle_{c 3}=\frac{\beta^{2}}{\sqrt{\gamma^{4}+\beta^{4}}}|V\rangle_{c 3}+\frac{\gamma^{2}}{\sqrt{\gamma^{4}+\beta^{4}}}|H\rangle_{c 3}
$$

Therefore, states $\left|\Psi_{2}^{+}\right\rangle_{d 1 b 1 c 1}^{\prime}$ and $\left|\Phi^{\prime}\right\rangle_{c 3}$ combined with the coherent state, $|\alpha\rangle$, evolve to:

$$
\begin{aligned}
\left|\Phi^{\prime}\right\rangle_{c 3} & \otimes\left|\Psi_{2}^{+}\right\rangle_{d 1 b 1 c 1}^{\prime}|\alpha\rangle \rightarrow \frac{\beta^{2} \gamma^{2}}{\sqrt{\gamma^{4}+2 \beta^{4}} \sqrt{\gamma^{4}+\beta^{4}}}|H\rangle_{d 1}|H\rangle_{b 1}|V\rangle_{c 1}|V\rangle_{e 2}\left|\alpha e^{i \theta}\right\rangle \\
& +\frac{\gamma^{4}}{\sqrt{\gamma^{4}+2 \beta^{4}} \sqrt{\gamma^{4}+\beta^{4}}}|H\rangle_{d 1}|H\rangle_{b 1}|V\rangle_{c 1}|H\rangle_{e 2}|\alpha\rangle \\
& +\frac{\beta^{4}}{\sqrt{\gamma^{4}+2 \beta^{4}} \sqrt{\gamma^{4}+\beta^{4}}}|V\rangle_{d 1}|H\rangle_{b 1}|H\rangle_{c 1}|V\rangle_{e 2}\left|\alpha e^{i 2 \theta}\right\rangle \\
& +\frac{\beta^{2} \gamma^{2}}{\sqrt{\gamma^{4}+2 \beta^{4}} \sqrt{\gamma^{4}+\beta^{4}}}|V\rangle_{d 1}|H\rangle_{b 1}|H\rangle_{c 1}|H\rangle_{e 2}\left|\alpha e^{i \theta}\right\rangle \\
& +\frac{\beta^{4}}{\sqrt{\gamma^{4}+2 \beta^{4}} \sqrt{\gamma^{4}+\beta^{4}}}|H\rangle_{d 1}|V\rangle_{b 1}|H\rangle_{c 1}|V\rangle_{e 2}\left|\alpha e^{i 2 \theta}\right\rangle \\
& +\frac{\beta^{2} \gamma^{2}}{\sqrt{\gamma^{4}+2 \beta^{4}} \sqrt{\gamma^{4}+\beta^{4}}}|H\rangle_{d 1}|V\rangle_{b 1}|H\rangle_{c 1}|H\rangle_{e 2}\left|\alpha e^{i \theta}\right\rangle
\end{aligned}
$$

After the photons in the spatial modes, $c 1$ and $c 3$, pass through the QND2, if the homodyne measurement of the coherent state is $\theta$, Equation (89) will also collapse to the maximally entangled $\mathrm{W}$ state. The success probability $P_{2}^{2}$ is:

$$
P_{2}^{2}=\frac{\gamma^{4}+2 \beta^{4}}{\left(\gamma^{2}+\beta^{2}\right)\left(\gamma^{2}+2 \beta^{2}\right)} \frac{3 \beta^{4} \gamma^{4}}{\left(\gamma^{4}+2 \beta^{4}\right)\left(\gamma^{4}+\beta^{4}\right)}=\frac{3 \beta^{4} \gamma^{4}}{\left(\gamma^{2}+2 \beta^{2}\right)\left(\gamma^{4}+\beta^{4}\right)\left(\gamma^{2}+\beta^{2}\right)}
$$

Here, the subscript " 2 " means the second concentration round.

In the first step, we calculate the success probability in the Nth round:

$$
P_{N}^{1}=\frac{\alpha^{2^{N}}\left(\beta^{2^{N}-2} \gamma^{2}+2 \beta^{2^{N}}\right)}{\left(\alpha^{2^{N}}+\beta^{2^{N}}\right)\left(\alpha^{2^{N-1}}+\beta^{2^{N-1}}\right) \cdots\left(\alpha^{2}+\beta^{2}\right)}
$$

In the second step, we obtain the success probability in the Mth round:

$$
P_{M}^{2}=\frac{3 \beta^{2^{M}} \gamma^{2^{M}}}{\left(\gamma^{2^{M}}+\beta^{2^{M}}\right)\left(\gamma^{2^{M-1}}+\beta^{2^{M-1}}\right) \cdots\left(\gamma^{2}+\beta^{2}\right)} \cdot \frac{1}{\left(\gamma^{2}+2 \beta^{2}\right)}
$$

Therefore, by repeating both steps, the total success probability is:

$$
\begin{aligned}
P_{\text {total }} & =P_{1}^{1}\left(P_{1}^{2}+P_{2}^{2}+\cdots+P_{M}^{2}\right)+P_{2}^{1}\left(P_{1}^{2}+P_{2}^{2}+\cdots+P_{M}^{2}\right) \\
& +\cdots+P_{N}^{1}\left(P_{1}^{2}+P_{2}^{2}+\cdots+P_{M}^{2}\right)=\sum_{N=1}^{\infty} P_{N}^{1} \sum_{M=1}^{\infty} P_{M}^{2}
\end{aligned}
$$

We calculate the total success probability of both the PBS and the QND protocol, as shown in Figure 14. In Figure 14, it is shown that both success probability monotones increase with $\alpha$, when $\alpha \in\left(0, \sqrt{\frac{1}{3}}\right)$. They both have a maximal value when $\alpha=\frac{1}{\sqrt{3}}$. 
Figure 14. The total success probability, $\mathrm{P}$, of getting a maximally entangled $\mathrm{W}$ state is altered with the initial coefficient, $\alpha$ [60]. Here, we choose $\beta=\frac{1}{\sqrt{3}}, \alpha \in\left(0, \sqrt{\frac{2}{3}}\right)$. Curve $\mathrm{A}$ is the ECP with QND, and Curve B is the ECP with PBS. For numerical simulation, we choose $N=M=3$ for approximation.

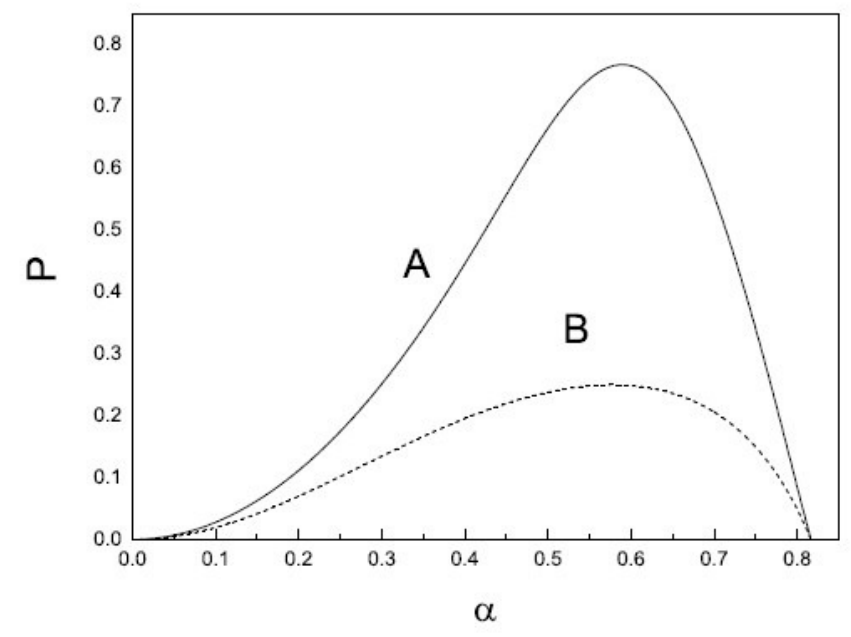

\subsection{Entanglement Concentration for Single-Photon Multi-Mode W State}

The single-photon Bell state, $\frac{1}{\sqrt{2}}\left(|1\rangle_{A}|0\rangle_{B}+|0\rangle_{A}|1\rangle_{B}\right)=\frac{1}{\sqrt{2}}\left(a^{\dagger}+b^{\dagger}\right)|0\rangle$, described in Section 2.3, can be easily extended to the single-photon multi-mode W state of the form [61]:

$$
|W\rangle_{N}=\frac{1}{\sqrt{N}}(|1,0,0, \cdots, 0\rangle+|0,1,0, \cdots, 0\rangle+\cdots+|0, \cdots 0,1\rangle)
$$

where the single photon is in the superposition of $\mathrm{N}$ spatial modes in different locations $(\mathrm{N}>2)$. Experimental schemes to generate these $\mathrm{W}$ states have been already proposed [62-65]. It has been proven that the $\mathrm{W}$ state is robust to decoherence in the noisy environment [66-68], and it displays an effective all-versus-nothing nonlocality, as the number of $\mathrm{N}$ delocalizations of the single particle goes up [69]. Especially, Gottesman et al. discussed an approach to building interferometric telescopes using such W states [70]. Unfortunately, in practical application, the maximally entangled W state may inevitably suffer decoherence under realistic conditions, which can make it degrade to the pure partially entangled state of the form:

$$
\left|W^{\prime}\right\rangle_{N}=a_{1}|1,0,0, \cdots, 0\rangle+a_{2}|0,1,0, \cdots, 0\rangle+\cdots+a_{N}|0, \cdots 0,1\rangle
$$

where $\left|a_{1}\right|^{2}+\left|a_{2}\right|^{2}+\cdots+\left|a_{N}\right|^{2}=1$, and all the $a_{i}(i=1,2, \cdots, N)$ are not equal.

In this section, we put forward an efficient ECP for recovering the single-photon three-mode partially entangled $\mathrm{W}$ state into the maximally entangled $\mathrm{W}$ state [61]. We suppose a single photon source, here named $S_{1}$, emits a photon and sends it to the three parties, say Alice, Bob and Charlie, in the spatial mode, $\mathrm{a}_{1}, \mathrm{~b}_{1}$ and $\mathrm{c}_{1}$, respectively. Therefore, a partially entangled three-mode $\mathrm{W}$ state shared by the three parties is created, which can be written as:

$$
|\psi\rangle_{a_{1} b_{1} c_{1}}=\alpha|1,0,0\rangle_{a_{1} b_{1} c_{1}}+\beta|0,1,0\rangle_{a_{1} b_{1} c_{1}}+\gamma|0,0,1\rangle_{a_{1} b_{1} c_{1}}
$$


$\alpha, \beta$ and $\gamma$ are the coefficients of the initial W state, where $|\alpha|^{2}+|\beta|^{2}+|\gamma|^{2}=1$ and $\alpha \neq \beta \neq \gamma$. The QND is shown in Figure 15, which is different from the QND as shown in Figures 1 and 3. The basic principle of the ECP is described in Figure 16.

Figure 15. A schematic drawing of the photon-number quantum nondemolition detector (QND) based on the weak cross-Kerr nonlinearity [61]. Here, the photons in the spatial modes, $\mathrm{a}_{1}$ and $\mathrm{a}_{2}$, pass through the cross-Kerr material. In the early work of [51], the high-efficiency quantum-nondemolition single-photon-number-resolving detector with cross-Kerr nonlinearity was proposed. This setup is a development of [51], for it is essentially the parity check measurement for the photon number.

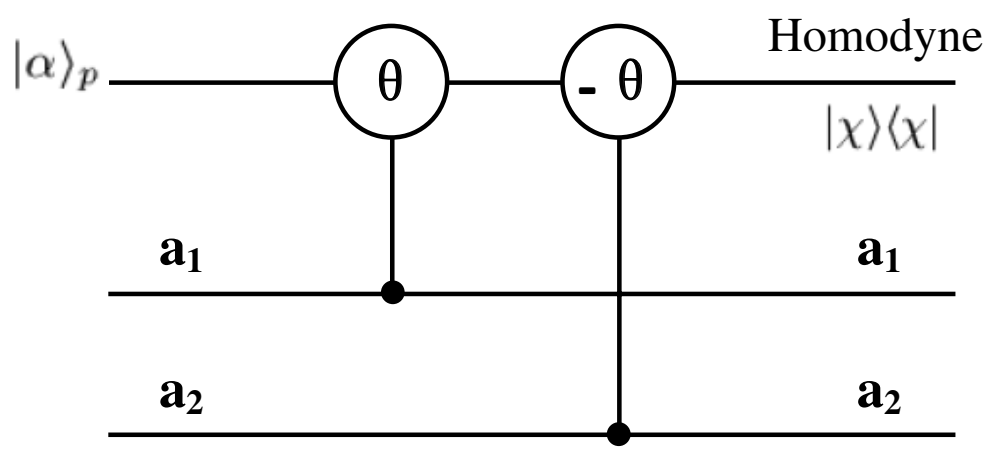

Figure 16. A schematic drawing of our ECP for distilling the single-photon maximally entangled $\mathrm{W}$ state from the arbitrary single-photon partially entangled $\mathrm{W}$ state [61]. The VBSis used to adjust the coefficients of the entangle state and, ultimately, obtain the maximally entangled state [71].

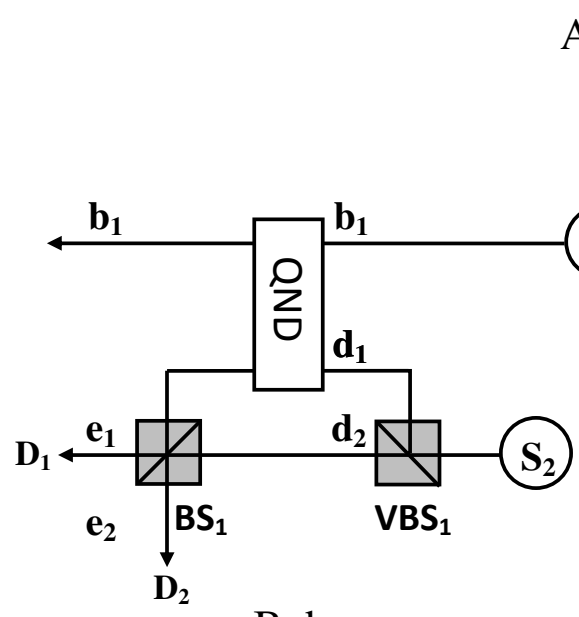

Bob
Alice

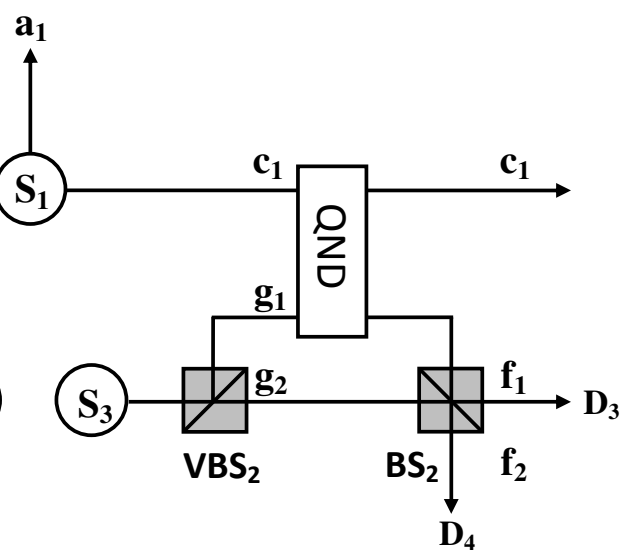

Charlie

Analogous to Section 3.1, the whole protocol can be divided into two steps. In the first step, the single photon source, here named $\mathrm{S}_{2}$, emits an auxiliary single photon and sends it to Bob. Then, the single photon is passed through the $\mathrm{VBS}_{1}$, with the transmission of $\mathrm{t}$ [71]. In this way, it can be written as:

$$
|\psi\rangle_{d_{1} d_{2}}=\sqrt{1-t}|1,0\rangle_{d_{1} d_{2}}+\sqrt{t}|0,1\rangle_{d_{1} d_{2}}
$$


Therefore, the whole two-photon system can be described as:

$$
\begin{aligned}
|\Psi\rangle_{a_{1} b_{1} c_{1} d_{1} d_{2}} & =|\psi\rangle_{a_{1} b_{1} c_{1}} \otimes|\psi\rangle_{d_{1} d_{2}} \\
& =(\alpha \sqrt{1-t}|1,0,0,1,0\rangle+\alpha \sqrt{t}|1,0,0,0,1\rangle \\
& +\beta \sqrt{1-t}|0,1,0,1,0\rangle+\beta \sqrt{t}|0,1,0,0,1\rangle \\
& +\gamma \sqrt{1-t}|0,0,1,1,0\rangle+\gamma \sqrt{t}|0,0,1,0,1\rangle)_{a_{1} b_{1} c_{1} d_{1} d_{2}}
\end{aligned}
$$

After the two photons pass through the QND, the whole system combined with the coherent state can evolve to:

$$
\begin{aligned}
|\Psi\rangle_{a_{1} b_{1} c_{1} d_{1} d_{2}} \otimes|\alpha\rangle & \longrightarrow \alpha \sqrt{1-t}|1,0,0,1,0\rangle_{a_{1} b_{1} c_{1} d_{1} d_{2}}\left|\alpha e^{-i \theta}\right\rangle+\alpha \sqrt{t}|1,0,0,0,1\rangle_{a_{1} b_{1} c_{1} d_{1} d_{2}}|\alpha\rangle \\
& +\beta \sqrt{1-t}|0,1,0,1,0\rangle_{a_{1} b_{1} c_{1} d_{1} d_{2}}|\alpha\rangle+\beta \sqrt{t}|0,1,0,0,1\rangle_{a_{1} b_{1} c_{1} d_{1} d_{2}}\left|\alpha e^{i \theta}\right\rangle \\
& +\gamma \sqrt{1-t}|0,0,1,1,0\rangle_{a_{1} b_{1} c_{1} d_{1} d_{2}}\left|\alpha e^{-i \theta}\right\rangle+\gamma \sqrt{t}|0,0,1,0,1\rangle_{a_{1} b_{1} c_{1} d_{1} d_{2}}|\alpha\rangle
\end{aligned}
$$

One can find that the items, $\alpha \sqrt{1-t}|1,0,0,1,0\rangle_{a_{1} b_{1} c_{1} d_{1} d_{2}}$ and $\gamma \sqrt{1-t}|0,0,1,1,0\rangle_{a_{1} b_{1} c_{1} d_{1} d_{2}}$, can make the coherent state pick up the phase shift of $-\theta$, while the item, $\beta \sqrt{t}|0,1,0,0,1\rangle_{a_{1} b_{1} c_{1} d_{1} d_{2}}$, can make it pick up $\theta$. After the measurements, Bob only selects the items corresponding to the phase shift, $\pm \theta$, and discards other items. Therefore, Equation (99) can collapse to:

$$
\begin{aligned}
\left|\Psi_{1}\right\rangle_{a_{1} b_{1} c_{1} d_{1} d_{2}} & =\alpha \sqrt{1-t}|1,0,0,1,0\rangle_{a_{1} b_{1} c_{1} d_{1} d_{2}}+\beta \sqrt{t}|0,1,0,0,1\rangle_{a_{1} b_{1} c_{1} d_{1} d_{2}} \\
& +\gamma \sqrt{1-t}|0,0,1,1,0\rangle_{a_{1} b_{1} c_{1} d_{1} d_{2}}
\end{aligned}
$$

Bob lets the photons in the spatial modes, $d_{1}$ and $d_{2}$, pass through the 50:50 beam splitter $\left(B_{1}\right)$, Equation (100) can evolve to:

$$
\begin{aligned}
\left|\Psi_{1}\right\rangle_{a_{1} b_{1} c_{1} e_{1} e_{2}} & =\left(\frac{\alpha \sqrt{1-t}}{\sqrt{2}}|1,0,0\rangle_{a_{1} b_{1} c_{1}}+\frac{\beta \sqrt{t}}{\sqrt{2}}|0,1,0\rangle_{a_{1} b_{1} c_{1}}+\frac{\gamma \sqrt{1-t}}{\sqrt{2}}|0,0,1\rangle_{a_{1} b_{1} c_{1}}\right)|1\rangle_{e_{1}} \\
& +\left(\frac{\alpha \sqrt{1-t}}{\sqrt{2}}|1,0,0\rangle_{a_{1} b_{1} c_{1}}-\frac{\beta \sqrt{t}}{\sqrt{2}}|0,1,0\rangle_{a_{1} b_{1} c_{1}}+\frac{\gamma \sqrt{1-t}}{\sqrt{2}}|0,0,1\rangle_{a_{1} b_{1} c_{1}}\right)|1\rangle_{e_{2}}
\end{aligned}
$$

From the above equation, it can become:

$$
\left|\psi_{1}\right\rangle_{a_{1} b_{1} c_{1}}=\alpha \sqrt{1-t}|1,0,0\rangle_{a_{1} b_{1} c_{1}}+\beta \sqrt{t}|0,1,0\rangle_{a_{1} b_{1} c_{1}}+\gamma \sqrt{1-t}|0,0,1\rangle_{a_{1} b_{1} c_{1}}
$$

if the detector, $\mathrm{D}_{1}$, fires. It can also become:

$$
\left|\psi_{1}^{\prime}\right\rangle_{a_{1} b_{1} c_{1}}=\alpha \sqrt{1-t}|1,0,0\rangle_{a_{1} b_{1} c_{1}}-\beta \sqrt{t}|0,1,0\rangle_{a_{1} b_{1} c_{1}}+\gamma \sqrt{1-t}|0,0,1\rangle_{a_{1} b_{1} c_{1}}
$$

if the detector, $\mathrm{D}_{2}$, fires. There is only a phase difference between Equation (102) and Equation (103). Interestingly, if we can adopt a suitable VBS with the transmission $t_{1}=\frac{|\alpha|^{2}}{|\alpha|^{2}+|\beta|^{2}}$, where the subscript " 1 " means in the first concentration step, Equation (102) can evolve to:

$$
\left|\psi_{1}\right\rangle_{a_{1} b_{1} c_{1}}=\frac{\alpha \beta}{\sqrt{\alpha^{2}+\beta^{2}}}|1,0,0\rangle_{a_{1} b_{1} c_{1}}+\frac{\alpha \beta}{\sqrt{\alpha^{2}+\beta^{2}}}|0,1,0\rangle_{a_{1} b_{1} c_{1}}+\frac{\gamma \beta}{\sqrt{\alpha^{2}+\beta^{2}}}|0,0,1\rangle_{a_{1} b_{1} c_{1}}(104
$$


Equation (104) can be rewritten as:

$$
\left|\psi_{1}\right\rangle_{a_{1} b_{1} c_{1}}=\alpha|1,0,0\rangle_{a_{1} b_{1} c_{1}}+\alpha|0,1,0\rangle_{a_{1} b_{1} c_{1}}+\gamma|0,0,1\rangle_{a_{1} b_{1} c_{1}}
$$

So far, we have completed the first concentration step. In this step, with the help of the cross-Kerr nonlinearities and the VBS, we successfully make the entanglement coefficients of the items $|1,0,0\rangle_{a_{1} b_{1} c_{1}}$ and $|0,1,0\rangle_{a_{1} b_{1} c_{1}}$ be the same, with the success probability of

$$
P_{1}^{1}=\frac{|\beta|^{2}\left(2|\alpha|^{2}+|\gamma|^{2}\right)}{|\alpha|^{2}+|\beta|^{2}}
$$

From the above discussion, we only pick up the cases that the phase shift is $\theta$. If Alice picks up no phase shift, the Equation (99) can collapse to:

$$
\begin{aligned}
\left|\Psi^{\prime}\right\rangle_{a_{1} b_{1} c_{1} d_{1} d_{2}} & =\alpha^{2}|1,0,0,0,1\rangle_{a_{1} b_{1} c_{1} d_{1} d_{2}}+\beta^{2}|0,1,0,1,0\rangle_{a_{1} b_{1} c_{1} d_{1} d_{2}} \\
& +\alpha \gamma|0,0,1,0,1\rangle_{a_{1} b_{1} c_{1} d_{1} d_{2}}
\end{aligned}
$$

With the same principle, it can be found that if the detector, $\mathrm{D}_{1}$, fires, Equation (107) will collapse to:

$$
\left|\psi_{2}\right\rangle_{a_{1} b_{1} c_{1}}=\alpha^{2}|1,0,0\rangle_{a_{1} b_{1} c_{1}}+\beta^{2}|0,1,0\rangle_{a_{1} b_{1} c_{1}}+\alpha \gamma|0,0,1\rangle_{a_{1} b_{1} c_{1}}
$$

while if the detector, $\mathrm{D}_{2}$, fires, Equation (107) will collapse to:

$$
\left|\psi_{2}^{\prime}\right\rangle_{a_{1} b_{1} c_{1}}=\alpha^{2}|1,0,0\rangle_{a_{1} b_{1} c_{1}}-\beta^{2}|0,1,0\rangle_{a_{1} b_{1} c_{1}}+\alpha \gamma|0,0,1\rangle_{a_{1} b_{1} c_{1}}
$$

Similarly, Equation (109) can be easily converted to Equation (108) by a phase flip operation from Bob. Equation (108) can be rewritten as:

$$
\left|\psi_{2}\right\rangle_{a_{1} b_{1} c_{1}}=\alpha^{\prime}|1,0,0\rangle_{a_{1} b_{1} c_{1}}+\beta^{\prime}|0,1,0\rangle_{a_{1} b_{1} c_{1}}+\gamma^{\prime}|1,0,0\rangle_{a_{1} b_{1} c_{1}}
$$

Here, $\alpha^{\prime}=\frac{\alpha^{2}}{\sqrt{\alpha^{4}+\beta^{4}+\alpha^{2} \gamma^{2}}}, \beta^{\prime}=\frac{\beta^{2}}{\sqrt{\alpha^{4}+\beta^{4}+\alpha^{2} \gamma^{2}}}$ and $\gamma^{\prime} \frac{\alpha \gamma}{\sqrt{\alpha^{4}+\beta^{4}+\alpha^{2} \gamma^{2}}}$. It has the same form of the initial partially entangled W state in Equation (96) and can be reconcentrated in the second round. Bob chooses another VBS with the transmission of $t_{1}^{2}$, where the subscript " 1 " means in the first concentration step and the superscript " 2 " means in the second concentration round. After the single photon passes through the VBS, the new two-photon system can ultimately evolve to:

$$
\left|\psi_{3}\right\rangle_{a_{1} b_{1} c_{1}}=\alpha^{2} \sqrt{1-t_{1}^{2}}|1,0,0\rangle_{a_{1} b_{1} c_{1}}+\beta^{2} \sqrt{t_{1}^{2}}|0,1,0\rangle_{a_{1} b_{1} c_{1}}+\alpha \gamma \sqrt{1-t_{1}^{2}}|0,0,1\rangle_{a_{1} b_{1} c_{1}}
$$

If a suitable transmission, $\mathrm{t}_{1}^{2}=\frac{\left|\alpha^{\prime}\right|^{2}}{\left|\alpha^{\prime}\right|^{2}+\left|\beta^{\prime}\right|^{2}}=\frac{|\alpha|^{4}}{|\alpha|^{4}+|\beta|^{4}}$, can be selected, Equation (111) can be converted into Equation (105), with the success probability of:

$$
P_{1}^{2}=\frac{|\beta|^{4}\left(|\alpha \gamma|^{2}+2|\alpha|^{4}\right)}{\left(|\alpha|^{2}+|\beta|^{2}\right)\left(|\alpha|^{4}+|\beta|^{4}\right)}
$$

Similarly, we can get a new partially entangled W state as:

$$
\left|\psi_{4}\right\rangle_{a_{1} b_{1} c_{1}}=\alpha^{4}|1,0,0\rangle_{a_{1} b_{1} c_{1}}+\beta^{4}|0,1,0\rangle_{a_{1} b_{1} c_{1}}+\alpha^{3} \gamma|0,0,1\rangle_{a_{1} b_{1} c_{1}}
$$


which can be reconcentrated for the third round. Following the same principle, Alice can perform the concentration step for $K$ times, with $\mathrm{t}_{1}^{K}=\frac{|\alpha|^{2^{K}}}{|\alpha|^{2^{K}}+|\beta|^{2^{K}}}$, with the success probability as:

$$
P_{1}^{K}=\frac{|\beta|^{2^{K}}\left(|\alpha|^{2^{K}-2}|\gamma|^{2}+2|\alpha|^{2^{K}}\right)}{\left(|\alpha|^{2}+|\beta|^{2}\right)\left(|\alpha|^{4}+|\beta|^{4}\right) \cdots\left(|\alpha|^{2^{K}}+|\beta|^{2^{K}}\right)}
$$

and the total success probability of the first concentration step can be written as:

$$
P_{\text {total } 1}=P_{1}^{1}+P_{1}^{2}+\cdots+P_{1}^{K}=\sum_{K=1}^{\infty} P_{1}^{K}
$$

The concentration step in Charlie's location is analogous to that of Alice. He first chooses a suitable VBS, named $\mathrm{VBS}_{2}$, and makes the single photon become:

$$
|\psi\rangle_{g_{1} g_{2}}=\sqrt{1-t_{2}}|1,0\rangle_{g_{1} g_{2}}+\sqrt{t_{2}}|0,1\rangle_{g_{1} g_{2}}
$$

The two photons combined with the coherent state can be written as:

$$
\begin{aligned}
\left|\psi_{1}\right\rangle_{a_{1} b_{1} c_{1}} & \otimes|\psi\rangle_{g_{1} g_{2}} \otimes|\alpha\rangle \rightarrow \alpha \sqrt{1-t_{2}}|1,0,0,1,0\rangle_{a_{1} b_{1} c_{1} g_{1} g_{2}}\left|\alpha e^{-i \theta}\right\rangle+\alpha \sqrt{t_{2}}|1,0,0,0,1\rangle_{a_{1} b_{1} c_{1} g_{1} g_{2}}|\alpha\rangle \\
& +\alpha \sqrt{1-t_{2}}|0,1,0,1,0\rangle_{a_{1} b_{1} c_{1} g_{1} g_{2}}\left|\alpha e^{i \theta}\right\rangle+\alpha \sqrt{t_{2}}|0,1,0,0,1\rangle_{a_{1} b_{1} c_{1} g_{1} g_{2}}|\alpha\rangle \\
& +\gamma \sqrt{1-t_{2}}|0,0,1,1,0\rangle_{a_{1} b_{1} c_{1} g_{1} g_{2}}|\alpha\rangle+\gamma \sqrt{t_{2}}|0,0,1,0,1\rangle_{a_{1} b_{1} c_{1} g_{1} g_{2}}\left|\alpha e^{i \theta}\right\rangle
\end{aligned}
$$

If Charlie selects the cases that the coherent state picks up the phase shift $\theta$, the above equation will become:

$$
\begin{aligned}
\left|\Psi_{2}\right\rangle_{a_{1} b_{1} c_{1} g_{1} g_{2}} & =\alpha \sqrt{1-t_{2}}|1,0,0,1,0\rangle_{a_{1} b_{1} c_{1} g_{1} g_{2}}+\alpha \sqrt{1-t_{2}}|0,1,0,1,0\rangle_{a_{1} b_{1} c_{1} g_{1} g_{2}} \\
& +\gamma \sqrt{t_{2}}|0,0,1,0,1\rangle_{a_{1} b_{1} c_{1} g_{1} g_{2}}
\end{aligned}
$$

After passing through the BS, it becomes:

$$
\left|\psi_{5}\right\rangle_{a_{1} b_{1} c_{1}}=\alpha \sqrt{1-t_{2}}|1,0,0\rangle_{a_{1} b_{1} c_{1}}+\alpha \sqrt{1-t_{2}}|0,1,0\rangle_{a_{1} b_{1} c_{1}}+\gamma \sqrt{t_{2}}|0,0,1\rangle_{a_{1} b_{1} c_{1}}
$$

if the detector, $\mathrm{D}_{3}$, fires, and becomes:

$$
\left|\psi_{5}^{\prime}\right\rangle_{a_{1} b_{1} c_{1}}=\alpha \sqrt{1-t_{2}}|1,0,0\rangle_{a_{1} b_{1} c_{1}}-\alpha \sqrt{1-t_{2}}|0,1,0\rangle_{a_{1} b_{1} c_{1}}+\gamma \sqrt{t_{2}}|0,0,1\rangle_{a_{1} b_{1} c_{1}}
$$

if the detector, $\mathrm{D}_{4}$, fires. Obviously, if a suitable $\mathrm{VBS}_{2}$ with $\mathrm{t}_{2}=\frac{|\alpha|^{2}}{|\alpha|^{2}+|\gamma|^{2}}$ can be selected, Equation (119) can evolve to:

$$
\left|\psi_{5}\right\rangle_{a_{1} b_{1} c_{1}}=\frac{1}{\sqrt{3}}\left(|1,0,0\rangle_{a_{1} b_{1} c_{1}}+|0,1,0\rangle_{a_{1} b_{1} c_{1}}+|0,0,1\rangle_{a_{1} b_{1} c_{1}}\right)
$$

which is the maximally entangled W state. The Equation (120) also can become the maximally entangled W state with a phase flip operation. The success probability is:

$$
P_{2}^{1}=\frac{3|\alpha|^{2}|\gamma|^{2}}{\left(|\alpha|^{2}+|\gamma|^{2}\right)\left(2|\alpha|^{2}+|\gamma|^{2}\right)}
$$

where the subscript " 2 " means the second concentration step and the superscript " 1 " means the first concentration round. 
If the coherent state picks up no phase shift, they can obtain:

$$
\begin{aligned}
\left|\Psi_{2}^{\prime}\right\rangle_{a_{1} b_{1} c_{1} g_{1} g_{2}} & =\alpha^{2}|1,0,0,0,1\rangle_{a_{1} b_{1} c_{1} g_{1} g_{2}}+\alpha^{2}|0,1,0,0,1\rangle_{a_{1} b_{1} c_{1} g_{1} g_{2}} \\
& +\gamma^{2}|0,0,1,1,0\rangle_{a_{1} b_{1} c_{1} g_{1} g_{2}}
\end{aligned}
$$

From Equation (117), it can also be used to concentrate to the maximally entangled state, with the suitable VBS with $\mathrm{t}_{2}^{2}=\frac{|\alpha|^{4}}{|\alpha|^{4}+|\gamma|^{4}}$. The success probability is:

$$
P_{2}^{2}=\frac{3|\alpha|^{4}|\gamma|^{4}}{\left(|\alpha|^{2}+|\gamma|^{2}\right)\left(|\alpha|^{4}+|\gamma|^{4}\right)\left(2|\alpha|^{2}+|\gamma|^{2}\right)}
$$

In the $\mathrm{K} t h$ round, Charlie chooses the $\mathrm{VBS}_{2}$ with $\mathrm{t}_{2}^{K^{\prime}}=\frac{|\alpha|^{2^{K^{\prime}}}}{|\alpha|^{2^{\prime}}+|\gamma|^{2^{\prime}}}$, where the superscript "K" means the iteration time. The second concentration step can be reused to get a higher success probability. The success probability of the second concentration step in each round can be written as:

$$
P_{2}^{K^{\prime}}=\frac{3|\alpha|^{2^{K^{\prime}}}|\gamma|^{2^{K^{\prime}}}}{\left(|\alpha|^{2}+|\gamma|^{2}\right)\left(|\alpha|^{4}+|\gamma|^{4}\right) \cdots\left(|\alpha|^{2^{K^{\prime}}}+|\gamma|^{2^{K^{\prime}}}\right)\left(2|\alpha|^{2}+|\gamma|^{2}\right)}
$$

and the total success probability of the second concentration step is:

$$
P_{\text {total } 2}=P_{2}^{1}+P_{2}^{2}+\cdots+P_{2}^{K^{\prime}}=\sum_{K^{\prime}=1}^{\infty} P_{2}^{K^{\prime}}
$$

We can calculate the total success probability of this ECP as:

$$
P_{\text {total }}=P_{\text {total } 1} P_{\text {total } 2}=\sum_{K, K^{\prime}=1}^{\infty} P_{1}^{K} P_{2}^{K^{\prime}}
$$

\section{Some other ECPs}

\subsection{Entanglement Concentration for NOON State}

Recently, a special quantum state, named the NOON state, has drawn great attention [72-76]. It can be used to study the violations of quantum realism in the well-known GHZ contradictions[77]. It also shows great sensitivity for optical interferometry over a coherent state [78,79]. It can approach the Heisenberg limit of $1 / N$ [80-82]. For N-photon interference, the de Broglie wavelength is $\lambda / N$. The $\mathrm{N}$-photon NOON state can be written as:

$$
|N O O N\rangle_{a b}=\frac{1}{\sqrt{2}}\left(|0, N\rangle_{a b}+|N, 0\rangle_{a b}\right)
$$

Certainly, the maximally entangled NOON state inevitably interacts with the environment. For instance, the variation of the path length may induce a phase shift and makes it become:

$$
\left|N O O N^{\prime}\right\rangle_{a b}=\frac{1}{\sqrt{2}}\left(|0, N\rangle_{a b}+e^{i N \triangle \varphi}|N, 0\rangle_{a b}\right)
$$

where the $\triangle \varphi$ comes from the fluctuation of the path length [75,76]. If we consider other noise, the more general form of the partially entangled NOON state can be described as:

$$
\left|N O O N^{\prime \prime}\right\rangle_{a b}=\alpha|0, N\rangle_{a b}+\beta|N, 0\rangle_{a b}
$$


where $|\alpha|^{2}+|\beta|^{2}=1$ and $\alpha \neq \beta$. If the maximally entangled state is polluted, we should concentrate it into a maximally entangled one with the form of Equation (128).

Essentially, the single-photon entanglement described in Section 2.3 is the simplest NOON state. In this section, we will described the entanglement concentration for the NOON state following the similar principle in Section 2.3 [83].

From Figure 17, it is rather analogous with Figure 11. From Figure 17, by choosing two copies of partially entangled NOON states with the form of Equation (130), the whole 2N-photon system can be described as:

$$
|\Phi\rangle_{a_{1} a_{2} b_{1} b_{2}}^{N}=\left(\alpha^{2}|0,0, N, N\rangle+\beta^{2}|N, N, 0,0\rangle+\alpha \beta|0, N, N, 0\rangle+\alpha \beta|N, 0,0, N\rangle\right)_{a_{1} a_{2} b_{1} b_{2}}(13
$$

After the photons passes through the cross-Kerr nonlinearity, the state of the whole system combined with the coherent state can evolve to:

$$
\begin{aligned}
& |\Phi\rangle_{a_{1} a_{2} b_{1} b_{2}}^{N} \otimes|\alpha\rangle \rightarrow\left(\alpha^{2}|0,0, N, N\rangle|\alpha\rangle+\beta^{2}|N, N, 0,0\rangle|\alpha\rangle\right. \\
+ & \left.\alpha \beta|0, N, N, 0\rangle\left|\alpha e^{N i \theta}\right\rangle+\alpha \beta|N, 0,0, N\rangle\left|\alpha e^{-N i \theta}\right\rangle\right)_{a_{1} a_{2} b_{1} b_{2}}
\end{aligned}
$$

Then, they select the items, which make the coherent state pick up the phase shift of $\pm N \theta$, so that Equation (132) will collapse to:

$$
\left|\Phi_{1}\right\rangle_{a_{1} a_{2} b_{1} b_{2}}^{N}=\frac{1}{\sqrt{2}}(|0, N, N, 0\rangle+|N, 0,0, N\rangle)_{a_{1} a_{2} b_{1} b_{2}}
$$

with the success probability of $P_{1}^{N}=2|\alpha \beta|^{2}$. In order to obtain the maximally entangled NOON state, they let the photons pass through the BS simultaneously. With the help of the creation operators, Equation (133) becomes:

$$
\left.\left.\left|\Phi_{1}^{\prime}\right\rangle_{a_{1} a_{2} b_{1} b_{2}}^{N}=\frac{1}{N \sqrt{2}}\left(\left|\left(\hat{a}_{2}^{\dagger}\right)^{N}\left(\hat{b}_{1}^{\dagger}\right)^{N}\right| 0\right\rangle+\left|\left(\hat{a}_{1}^{\dagger}\right)^{N}\left(\hat{b}_{2}^{\dagger}\right)^{N}\right| 0\right\rangle\right)_{a_{1} a_{2} b_{1} b_{2}}
$$

Equation (134) can finally evolve to:

$$
\begin{aligned}
\left|\Phi_{1}\right\rangle_{a_{1} b_{1} d_{1} d_{2}}^{N} & =\frac{1}{\sqrt{2}(N+1)}\left(|N, 0, N, 0\rangle_{a_{1} b_{1} d_{1} d_{2}}+|0, N, N, 0\rangle_{a_{1} b_{1} d_{1} d_{2}}\right) \\
& +\frac{1}{\sqrt{2}^{(N+1)} \sqrt{N}} \sum_{i=1}^{N} \sqrt{i(N-i)} C_{N}^{i}\left[|N, 0,(N-i), i\rangle_{a_{1} b_{1} d_{1} d_{2}}\right. \\
& \left.+(-1)^{i}|0, N,(N-i), i\rangle_{a_{1} b_{1} d_{1} d_{2}}\right]
\end{aligned}
$$

If the photon number detected by $\mathrm{D}_{2}$ is even, Equation (135) will collapses to:

$$
|\Psi\rangle_{a_{1} b_{1}}^{N}=\frac{1}{\sqrt{2}}\left(|0, N\rangle_{a_{1} b_{1}}+|N, 0\rangle_{a_{1} b_{1}}\right)
$$

while if the photon number detected by $\mathrm{D}_{2}$ is odd, it will collapse to:

$$
\left|\Psi^{\prime}\right\rangle_{a_{1} b_{1}}^{N}=\frac{1}{\sqrt{2}}\left(|0, N\rangle_{a_{1} b_{1}}-|N, 0\rangle_{a_{1} b_{1}}\right)
$$

Both Equation (136) and Equation (137) are maximally entangled N-photon NOON states, and there is only a phase difference between them. 
Figure 17. A schematic drawing of the ECP for the N-photon partially entangled NOON state [83].

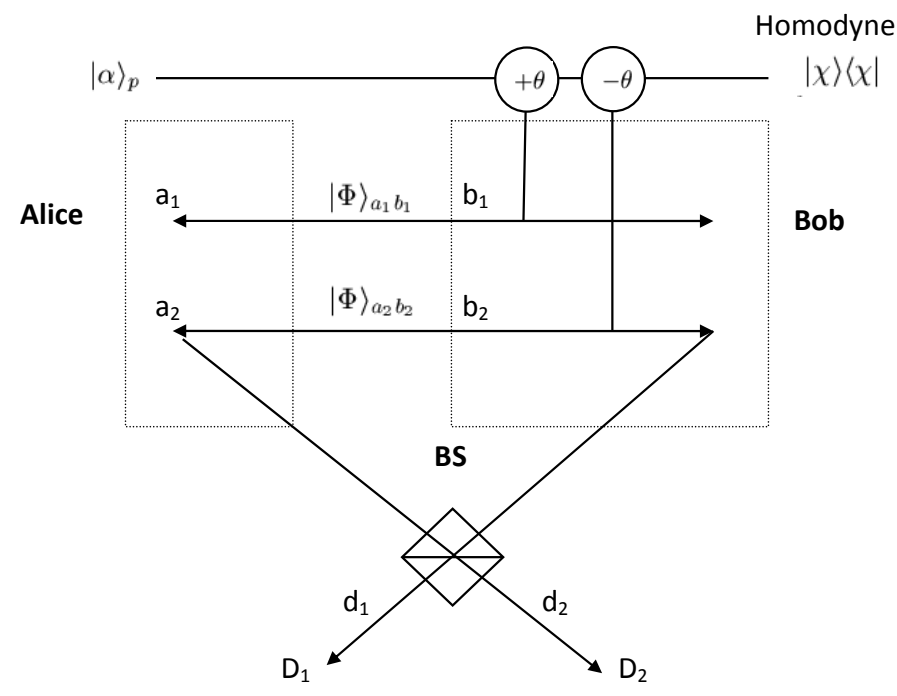

In order to obtain Equation (136), one of the two parties only needs to perform a phase flip operation on her or his photons to convert Equation (137) to Equation (136). The success probability is $P_{1}^{N}=$ $2|\alpha \beta|^{2}$.

On the other hand, if the previous step is a failure, which makes the coherent state pick up no phase shift, the remaining state is:

$$
\left|\Phi_{2}\right\rangle_{a_{1} a_{2} b_{1} b_{2}}^{N}=\alpha^{2}|0,0, N, N\rangle_{a_{1} a_{2} b_{1} b_{2}}+\beta^{2}|N, N, 0,0\rangle_{a_{1} a_{2} b_{1} b_{2}}
$$

By making the photons in modes $\mathrm{a}_{2}$ and $\mathrm{b}_{2}$ pass through the BS, Equation (138) can evolve to:

$$
\begin{aligned}
\left|\Phi_{2}\right\rangle_{a_{1} b_{1} d_{1} d_{2}}^{N} & \rightarrow \frac{\alpha^{2}}{\sqrt{2}^{N}}|0, N, N, 0\rangle_{a_{1} b_{1} d_{1} d_{2}}+\frac{\beta^{2}}{\sqrt{2}^{N}}|N, 0, N, 0\rangle_{a_{1} b_{1} d_{1} d_{2}} \\
& +\frac{1}{\sqrt{2}^{N} \sqrt{N}} \sum_{i=1}^{N} \sqrt{i(N-i)} C_{N}^{i}\left[\alpha^{2}|N, 0,(N-i), i\rangle_{a_{1} b_{1} d_{1} d_{2}}\right. \\
& \left.+(-1)^{i} \beta^{2}|0, N,(N-i), i\rangle_{a_{1} b_{1} d_{1} d_{2}}\right]
\end{aligned}
$$

With the same principle, if the photon number detected by $\mathrm{D}_{2}$ is even, Equation (139) will collapse to:

$$
\left|\Psi_{2}\right\rangle_{a_{1} b_{1}}^{N}=\alpha^{2}|0, N\rangle_{a_{1} b_{1}}+\beta^{2}|N, 0\rangle_{a_{1} b_{1}}
$$

If the photon number detected by $\mathrm{D}_{2}$ is odd, Equation (139) collapses to:

$$
\left|\Psi_{2}^{\prime}\right\rangle_{a_{1} b_{1}}^{N}=\alpha^{2}|0, N\rangle_{a_{1} b_{1}}-\beta^{2}|N, 0\rangle_{a_{1} b_{1}}
$$

From Equation (140) and Equation (141), they are both the partially entangled N-photon NOON states. Equation (141) can be converted to Equation (140) by performing the phase flip operation. Equation (140) has the same form with Equation (130), and following the same principle, it can be reconcentrated in the third round. In this way, we have proven that our ECP can be used repeatedly to further concentrate arbitrary N-photon NOON states. 


\subsection{Entanglement Concentration for Electrons with Charge Detection}

Quantum communication and computation can also be achieved with conduction electrons, since Beenakker et al. broke through the obstacle of the no-go theorem [84,85]. In 2004, with the help of a charge detector, they proposed a deterministic CNOT gate based on the spin degree of freedom [84]. The charge detector can distinguish the occupation number, 1 , from the occupation number, 0 and 2 , but cannot distinguish between 0 and 2. Interestingly, spin and charge commute, so that the measurement of the charge leaves the spin qubit unaffected. Based on such elements, several quantum information processing protocols were proposed [86-94].

The ECP for electrons with charge detection is rather analogous with the ECP described in Section 2. As shown in Figure 18, there are two pairs of entangled electrons in the following unknown polarization states [94]:

$$
\begin{aligned}
& |\Phi\rangle_{a_{1} b_{1}}=\alpha|\uparrow\rangle_{a_{1}}|\uparrow\rangle_{b_{1}}+\beta|\downarrow\rangle_{a_{1}}|\downarrow\rangle_{b_{1}} \\
& |\Phi\rangle_{a_{2} b_{2}}=\alpha|\uparrow\rangle_{a_{2}}|\uparrow\rangle_{b_{2}}+\beta|\downarrow\rangle_{a_{2}}|\downarrow\rangle_{b_{2}}
\end{aligned}
$$

where $|\alpha|^{2}+|\beta|^{2}=1$. The $|\uparrow\rangle$ is spin up, and $|\downarrow\rangle$ is spin down. After the state passes through the half-wave plates, which are used to transfer the $|\uparrow\rangle$ to $|\downarrow\rangle$ and vice versa, the whole state becomes:

$$
\begin{aligned}
|\Psi\rangle^{\prime} & =\alpha^{2}|\uparrow\rangle_{a_{1}}|\downarrow\rangle_{a_{3}}|\uparrow\rangle_{b_{1}}|\downarrow\rangle_{b_{3}}+\alpha \beta|\uparrow\rangle_{a_{1}}|\downarrow\rangle_{a_{3}}|\uparrow\rangle_{b_{1}}|\uparrow\rangle_{b_{3}} \\
& +\alpha \beta|\downarrow\rangle_{a_{1}}|\downarrow\rangle_{a_{3}}|\downarrow\rangle_{b_{1}}|\downarrow\rangle_{b_{3}}+\beta^{2}|\downarrow\rangle_{a_{1}}|\uparrow\rangle_{a_{3}}|\downarrow\rangle_{b_{1}}|\uparrow\rangle_{b_{3}}
\end{aligned}
$$

The PBS for the electron can transmit the spin up and reflect $|\uparrow\rangle$ the spin down $|\downarrow\rangle$. If the charge detector detects only one electron, the original state will collapse to:

$$
|\Psi\rangle^{\prime \prime}=\frac{1}{\sqrt{2}}\left(|\uparrow\rangle_{a_{1}}|\uparrow\rangle_{a_{3}}|\uparrow\rangle_{b_{1}}|\uparrow\rangle_{b_{3}}+|\downarrow\rangle_{a_{1}}|\downarrow\rangle_{a_{3}}|\downarrow\rangle_{b_{1}}|\downarrow\rangle_{b_{3}}\right)
$$

It is easy to get a two-particle maximally entangled state from Equation (144). They only need to use the Hadamard operation to change $a_{3} b_{3}$ to the x-axis. Finally, by measuring the electrons, $a_{4} c_{3}$, with the basis, $\mathrm{Z}$; if the two detectors, $D_{1}$ and $D_{2}$, have the same results, the $a_{1} b_{1}$ will collapse to the state:

$$
\left|\phi^{+}\right\rangle_{a_{1} b_{1}}=\frac{1}{\sqrt{2}}\left(|\uparrow\rangle_{a_{1}}|\uparrow\rangle_{b_{1}}+|\downarrow\rangle_{a_{1}}|\downarrow\rangle_{b_{1}}\right)
$$

Otherwise, thet will get:

$$
\left|\phi^{-}\right\rangle_{a_{1} b_{1}}=\frac{1}{\sqrt{2}}\left(|\uparrow\rangle_{a_{1}}|\uparrow\rangle_{b_{1}}-|\downarrow\rangle_{a_{1}}|\downarrow\rangle_{b_{1}}\right)
$$

In this ECP, the charge detector is used to detect the parity of the two electrons. If the two electrons are in even parity (both spin up or down), the charge detector will detect only one electron, and this above state can be concentrated to the maximally entangled state. Otherwise, the charge detector will detect zero or two electrons, which will make the state collapse to another partially entangled state as:

$$
\left|\Phi_{1}\right\rangle^{\prime \prime}=\alpha^{2}|\uparrow\rangle_{a_{1}}|\downarrow\rangle_{a_{3}}|\uparrow\rangle_{b_{1}}|\downarrow\rangle_{b_{3}}+\beta^{2}|\downarrow\rangle_{a_{1}}|\uparrow\rangle_{a_{3}}|\downarrow\rangle_{b_{1}}|\uparrow\rangle_{b_{3}}
$$


For $\left|\Phi_{1}\right\rangle^{\prime \prime}$, it can be concentrated to the maximally entangled state in the next round. As shown in Figure 19, after the measurements, Equation (147) becomes:

$$
\left|\Phi_{1}\right\rangle^{\prime \prime}=\alpha^{2}|\uparrow\rangle_{a_{1}}|\uparrow\rangle_{b_{1}} \pm \beta^{2}|\downarrow\rangle_{a_{1}}|\downarrow\rangle_{b_{1}}
$$

The "+" or "-" depends on the measurement results of $D_{1}$ and $D_{2}$. If the results of $D_{1}$ and $D_{2}$ are the same, both $|\uparrow \uparrow\rangle$ or $|\downarrow \downarrow\rangle$, they will get "+". Otherwise, they will get "-". Both of them are the partially entangled state and can be reconcentrated in the next round.

Figure 18. Schematic diagram of the proposed electronic ECP. Alice and Bob receive two pairs of identical less entangled photons, which are sent from source $S_{1}$ and $S_{2}$ [94]. The PBS plus the charge detector can make a parity check for the spin of electrons. The Hadamard operation is to change the spin in the $\mathrm{z}$-axis to the $\mathrm{x}$-axis.

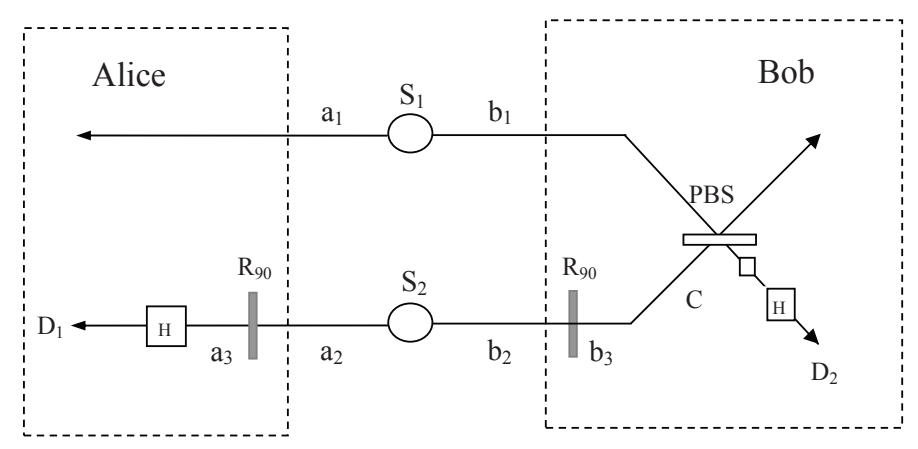

Figure 19. Another PBS is added in this protocol to make sure each mode contains only one electron. The device in Bob's laboratory is a complete parity measurement [84]. This protocol can be iterated and reach a higher yield and efficiency [94].

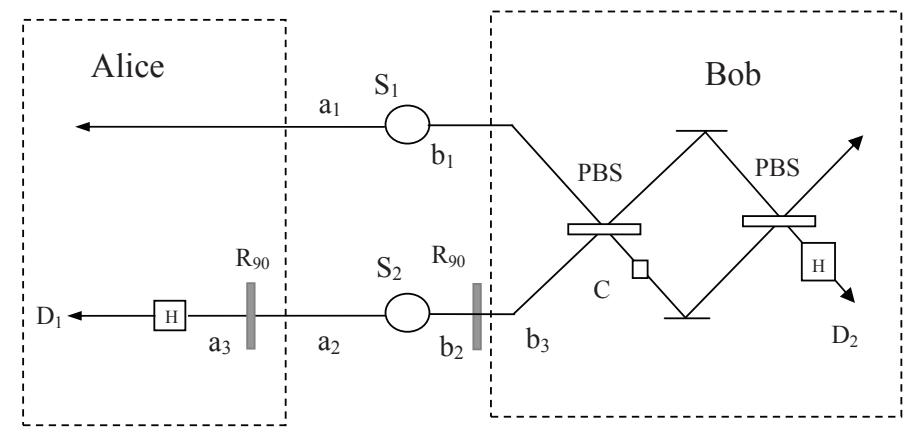

Certainly, the above ECP can also be improved, if we know the initial coefficients of the partially entangled state. We only need to use the single mobile electron to complete the entanglement concentration. The basic principle is shown in Figure 20. The basic idea is essentially similar to the optical ECP in Section 2.2. We only need to prepare the single electron of the form [90]:

$$
|\Phi\rangle_{b 2}=\alpha|\uparrow\rangle_{b 2}+\beta|\downarrow\rangle_{b 2}
$$

to start the protocol. Certainly, it can also be repeated if the first step is a failure, as discussed above. It can reach the same success probability as the ECP for photons. 
Figure 20. The schematic drawing of the principle for reconstructing the ECP [90]. If the measurement result of the charge detector is 0 , the remaining lesser-entangled pair can also be reused to perform the entanglement concentration. Another PBS, say $\mathrm{PBS}_{2}$, is used to couple the state into the same spatial mode. $\mathrm{P}$ denotes that it plays essentially the role of the parity check gate.

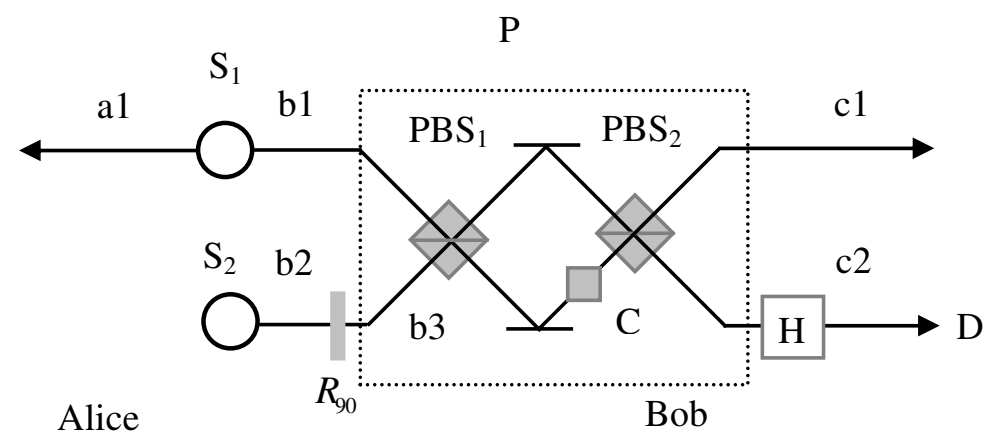

\subsection{Efficient Entanglement Concentration for Quantum Dot and Optical Microcavities Systems}

A charged quantum-dot carrying a single spin coupled to an optical microcavity is a novel system for quantum information processing [95]. In this system, the photon-spin entangling gate can be realized theoretically [96]. It is also shown that the neutral quantum-dot cavity systems behave like a beam splitter in the limit of the weak incoming field [97]. In 2009, Waks and Monroe described a scheme of creating hybrid entanglement between atomic quantum systems and semiconductors [95]. Entanglement purification and concentration protocols based on the hybrid entangled state using quantum-dot and microcavity coupled system were also proposed [100].

In the entanglement concentration, Wang et al. proposed an ECP for electron-spin entangled states using quantum-dot spins in optical microcavities in 2011 [100]. In this ECP, two pairs of partially entangled states and the ancillary photons are required. In 2012, He improved the ECP [101]. With the assistance of an ancillary quantum dot and a single photon, it can reach the same success probability. However, the second ECP of Wang et al. is still not an optimal one. In this section, we will describe the efficient ECP for such entangled electrons with the help of only one single photon [102]. Compared with the ECPs of Wang et al., the most significant advantage is that during the whole ECP, the single photon only needs to pass through one microcavity, which will increase the total success probability if the cavity is imperfect. Before we start to explain this ECP, we first describe the basic element of the ECP, say, the hybrid parity check gate, as shown in Figure 21. In the single charged GaAs/InAs quantum dot associated with the dipole transitions, it has four relevant electronic levels. There are two optically allowed transitions between the electron state and the exciton state, the bound state of two electrons and a hole. The spin of the holes are $J_{z}= \pm 3 / 2(|\Downarrow\rangle|\Uparrow\rangle)$, and the spin of the single electron states are $J_{z}= \pm 1 / 2(|\downarrow\rangle|\uparrow\rangle)$. The electron-spin state does not interact with the hole spin, because the total spin is zero, according to Pauli's principle. If the spin of the electron is in spin up, $|\uparrow\rangle$, and a photon is in state, $s_{z}=+1\left(\left|R^{\uparrow}\right\rangle\right.$ and $\left.\left|L^{\downarrow}\right\rangle\right)$, the circularly polarized light might change their polarization according to the direction of propagation, as well as the spin of the electron, after the photon passes through the cavity. 
Figure 21. A schematic drawing of the basic element of the ECP [102]. The quantum dot spin is coupled in optical microcavities. Input represents the input port of a photon. Output 1 and Output 2 are the output ports of the photon after coupled with the electron-spin system.

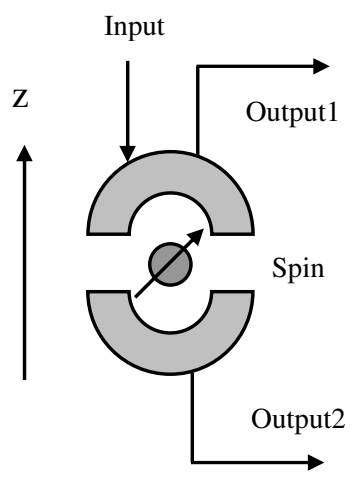

Figure 22. A schematic drawing of the basic principle of the ECP for quantum dot and optical microcavities systems [102]. The input mode means that the photon enters into the cavity. $\mathrm{D}_{1}, \mathrm{D}_{2}, \mathrm{D}_{3}$ and $\mathrm{D}_{4}$ are four single photon detectors. The half wave plate $\left(\mathrm{HWP}_{45}\right)$ can make a Hadamard operation.

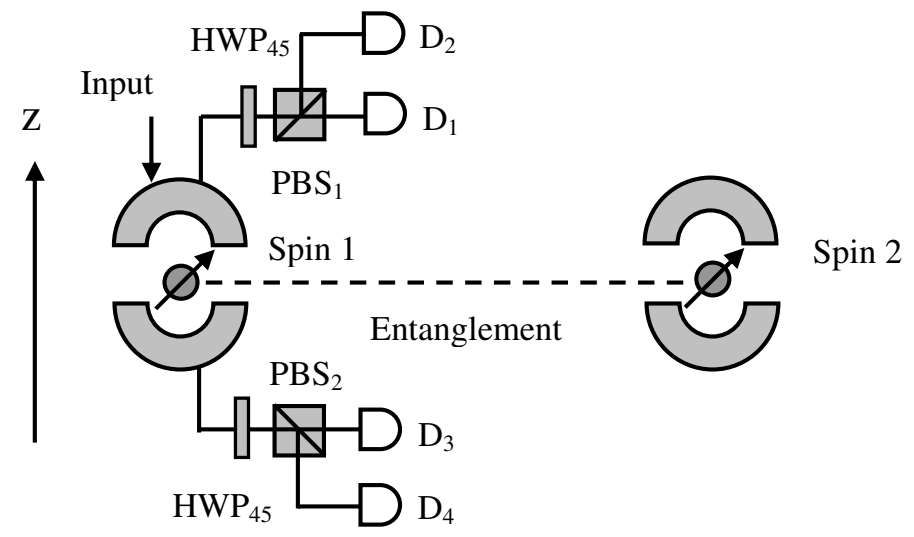

Alice

Bob

The total rules of the state change under the interaction of the photon with $s_{z}=+1\left(\left|R^{\uparrow}\right\rangle\right.$ and $\left.\left|L^{\downarrow}\right\rangle\right)$ and $s_{z}=-1\left(\left|R^{\downarrow}\right\rangle\right.$ and $\left.\left|L^{\uparrow}\right\rangle\right)$ can be described as [96,100]:

$$
\begin{array}{ll}
\left|R^{\uparrow}, \uparrow\right\rangle \rightarrow\left|L^{\downarrow}, \uparrow\right\rangle, & \left|R^{\downarrow}, \uparrow\right\rangle \rightarrow-\left|R^{\downarrow}, \uparrow\right\rangle \\
\left|R^{\uparrow}, \downarrow\right\rangle \rightarrow-\left|R^{\uparrow}, \downarrow\right\rangle, & \left|R^{\downarrow}, \downarrow\right\rangle \rightarrow\left|L^{\uparrow}, \downarrow\right\rangle \\
\left|L^{\uparrow}, \uparrow\right\rangle \rightarrow-\left|L^{\uparrow}, \uparrow\right\rangle, & \left|L^{\downarrow}, \uparrow\right\rangle \rightarrow\left|R^{\uparrow}, \uparrow\right\rangle \\
\left|L^{\uparrow}, \downarrow\right\rangle \rightarrow\left|R^{\downarrow}, \downarrow\right\rangle, & \left|L^{\downarrow}, \downarrow\right\rangle \rightarrow-\left|L^{\downarrow}, \downarrow\right\rangle
\end{array}
$$

Here, $|R\rangle$ and $|L\rangle$ denote the states of right-circular-polarized and left-circular-polarized photons, respectively. The $\uparrow$ and $\downarrow$ on the superscript arrow are the propagation direction along the z-axis, as shown in Figure 21. If the initial input state is the photon-spin product state, $\frac{1}{\sqrt{2}}(|R\rangle+|L\rangle) \otimes \frac{1}{\sqrt{2}}(\mid \uparrow$ \rangle$+|\downarrow\rangle)$, it will be converted into the two constituent hybrid entangled states, $\frac{1}{\sqrt{2}}(|R\rangle|\uparrow\rangle+|L\rangle|\downarrow\rangle)$, in the transmission port, say, output2 mode, and $\frac{1}{\sqrt{2}}(|R\rangle|\downarrow\rangle+|L\rangle|\uparrow\rangle)$ in the reflection port, say, output1 mode, with a success probability of $100 \%$, in principle. 
The initial partially entangled state shared by Alice and Bob is:

$$
\left|\phi^{+}\right\rangle_{12}=\alpha|\uparrow\rangle_{1}|\uparrow\rangle_{2}+\beta|\downarrow\rangle_{1}|\downarrow\rangle_{2}
$$

with $|\alpha|^{2}+|\beta|^{2}=1$. The subscripts "1" and "2" are spin 1 and spin 2 shown in Figure 22. Alice prepares another single photon as:

$$
|\Phi\rangle_{P}=\alpha|R\rangle+\beta|L\rangle
$$

The whole system evolves as:

$$
\begin{aligned}
& |\Phi\rangle_{P}\left|\phi^{+}\right\rangle_{12}=\left(\alpha\left|R^{\downarrow}\right\rangle+\beta\left|L^{\downarrow}\right\rangle\right)\left(\alpha|\uparrow\rangle_{1}|\uparrow\rangle_{2}+\beta|\downarrow\rangle_{1}|\downarrow\rangle_{2}\right) \\
= & \alpha^{2}\left|R^{\downarrow}\right\rangle|\uparrow\rangle_{1}|\uparrow\rangle_{2}+\beta^{2}\left|L^{\downarrow}\right\rangle|\downarrow\rangle_{1}|\downarrow\rangle_{2}+\alpha \beta\left(\left|R^{\downarrow}\right\rangle|\downarrow\rangle_{1}|\downarrow\rangle_{2}+\left|L^{\downarrow}\right\rangle|\uparrow\rangle_{1}|\uparrow\rangle_{2}\right) \\
\rightarrow & -\alpha^{2}\left|R^{\downarrow}\right\rangle|\uparrow\rangle_{1}|\uparrow\rangle_{2}-\beta^{2}\left|L^{\downarrow}\right\rangle|\downarrow\rangle_{1}|\downarrow\rangle_{2}+\alpha \beta\left(\left|L^{\uparrow}\right\rangle|\downarrow\rangle_{1}|\downarrow\rangle_{2}+\left|R^{\uparrow}\right\rangle|\uparrow\rangle_{1}|\uparrow\rangle_{2}\right)
\end{aligned}
$$

From the above description, if the photon is reflected and finally passes through the $\mathrm{HWP}_{45}$, Equation (153) will become:

$$
\begin{aligned}
& \frac{1}{\sqrt{2}}\left(\left|L^{\uparrow}\right\rangle|\downarrow\rangle_{1}|\downarrow\rangle_{2}+\left|R^{\uparrow}\right\rangle|\uparrow\rangle_{1}|\uparrow\rangle_{2}\right) \\
\rightarrow & \frac{1}{2}(|H\rangle-|V\rangle)|\downarrow\rangle_{1}|\downarrow\rangle_{2}+\frac{1}{2}(|H\rangle+|V\rangle)|\uparrow\rangle_{1}|\uparrow\rangle_{2} \\
= & \frac{1}{\sqrt{2}}|H\rangle \frac{1}{\sqrt{2}}\left(|\uparrow\rangle_{1}|\uparrow\rangle_{2}+|\downarrow\rangle_{1}|\downarrow\rangle_{2}\right)+\frac{1}{\sqrt{2}}|V\rangle \frac{1}{\sqrt{2}}\left(|\uparrow\rangle_{1}|\uparrow\rangle_{2}-|\downarrow\rangle_{1}|\downarrow\rangle_{2}\right)
\end{aligned}
$$

They will obtain $\frac{1}{\sqrt{2}}\left(|\uparrow\rangle_{1}|\uparrow\rangle_{2}+|\downarrow\rangle_{1}|\downarrow\rangle_{2}\right)$, if $D_{1}$ fires, and obtain $\frac{1}{\sqrt{2}}\left(|\uparrow\rangle_{1}|\uparrow\rangle_{2}-|\downarrow\rangle_{1}|\downarrow\rangle_{2}\right)$, if $D_{2}$ fires. The success probability is: $2|\alpha \beta|^{2}$.

Figure 23. Success probability, $P$, for obtaining a maximally entangled state after performing this ECP is altered with the initial coefficient, $\alpha \in(0,1)$ [102]. Curve A is the ideal case with no leakage. Curve $\mathrm{B}$ is the success probability with $\kappa_{s}=0.5 \kappa, g=0.5 \kappa$ and $\gamma=0.5 \kappa$ in this protocol. Curve $\mathrm{C}$ is the success probability of ECP in [101]. For numerical simulation, we let $K=5$ as a good approximation.

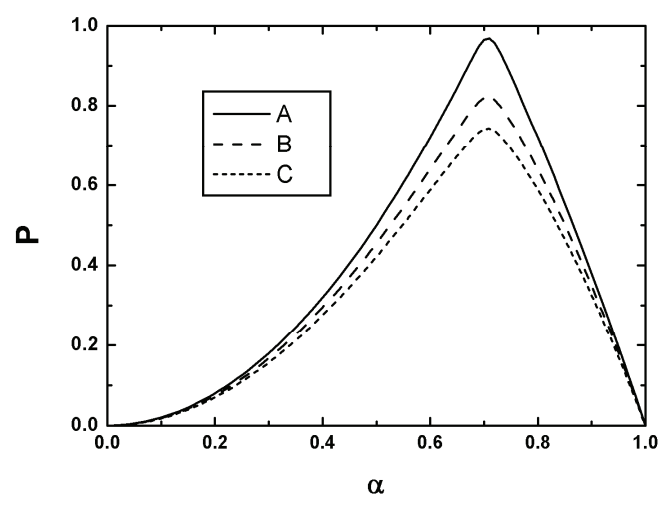

Certainly, from Equation (153), the photon may also be transmitted to another output mode and lead the state collapse to $\alpha^{2}\left|R^{\downarrow}\right\rangle|\uparrow\rangle_{1}|\uparrow\rangle_{2}+\beta^{2}\left|L^{\downarrow}\right\rangle|\downarrow\rangle_{1}|\downarrow\rangle_{2}$. By measuring the photon after passing through the $\mathrm{HWP}_{45}$ and $\mathrm{PBS}_{2}$, they will obtain:

$$
\left|\phi^{ \pm}\right\rangle_{12}^{\prime}=\alpha^{2}|\uparrow\rangle_{1}|\uparrow\rangle_{2} \pm \beta^{2}|\downarrow\rangle_{1}|\downarrow\rangle_{2}
$$


Both $\left|\phi^{ \pm}\right\rangle_{12}^{\prime}$ are lesser-entangled states and can be reconcentrated in the second round. If they obtain $\left|\phi^{+}\right\rangle_{12}^{\prime}$, Alice only needs to prepare another single photon of the form:

$$
|\Phi\rangle_{P}^{\prime}=\alpha^{2}|R\rangle+\beta^{2}|L\rangle
$$

In this way, the whole system can be written as:

$$
\begin{aligned}
& \left|\phi^{+}\right\rangle_{12}^{\prime}|\Phi\rangle_{P}^{\prime}=\left(\alpha^{2}|\uparrow\rangle_{1}|\uparrow\rangle_{2}+\beta^{2}|\downarrow\rangle_{1}|\downarrow\rangle_{2}\right)\left(\alpha^{2}\left|R^{\downarrow}\right\rangle+\beta^{2}\left|L^{\downarrow}\right\rangle\right) \\
= & \alpha^{4}|\uparrow\rangle_{1}|\uparrow\rangle_{2}\left|R^{\downarrow}\right\rangle+\beta^{4}|\downarrow\rangle_{1}|\downarrow\rangle_{2}\left|L^{\downarrow}\right\rangle+\alpha^{2} \beta^{2}\left(|\uparrow\rangle_{1}|\uparrow\rangle_{2}\left|L^{\downarrow}\right\rangle+|\downarrow\rangle_{1}|\downarrow\rangle_{2}\left|R^{\downarrow}\right\rangle\right) \\
\rightarrow & -\alpha^{4}\left|R^{\downarrow}\right\rangle|\uparrow\rangle_{1}|\uparrow\rangle_{2}-\beta^{4}\left|L^{\downarrow}\right\rangle|\downarrow\rangle_{1}|\downarrow\rangle_{2}+\alpha^{2} \beta^{2}\left(\left|L^{\uparrow}\right\rangle|\downarrow\rangle_{1}|\downarrow\rangle_{2}+\left|R^{\uparrow}\right\rangle|\uparrow\rangle_{1}|\uparrow\rangle_{2}\right)
\end{aligned}
$$

Obviously, from Equation (157), if the detector $\mathrm{D}_{1}$ or $\mathrm{D}_{2}$ fires, they will obtain the maximally entangled pair. If $\mathrm{D}_{1}$ or $\mathrm{D}_{2}$ fires, they will obtain another partially entangled pair:

$$
\left|\phi^{ \pm}\right\rangle_{12}^{\prime \prime}=\alpha^{4}|\uparrow\rangle_{1}|\uparrow\rangle_{2} \pm \beta^{4}|\downarrow\rangle_{1}|\downarrow\rangle_{2}
$$

It is still a partially entangled state, which can be reconcentrated for the third round. The success probability in the Kth round can be written as:

$$
P_{K}=\frac{2|\alpha \beta|^{2^{K}}}{\left(|\alpha|^{4}+|\beta|^{4}\right)\left(|\alpha|^{8}+|\beta|^{8}\right) \cdots\left(|\alpha|^{2^{K}}+|\beta|^{2^{k}}\right)}
$$

Actually, the realization of this ECP relies on the efficiency of transmission and the reflection for electrons and photon. By solving the Heisenberg equations of motion for the cavity-field operator and the trion dipole operator in weak excitation approximation, the reflection and transmission coefficients can be described as:

$$
\begin{aligned}
r(w) & =1+t(\omega) \\
t(\omega) & =\frac{-\kappa\left[i\left(\omega_{X^{-}}-\omega\right)+\frac{\gamma}{2}\right]}{\left[i\left(\omega_{X^{-}}-\omega\right)+\frac{\gamma}{2}\right]\left[i\left(\omega_{c}-\omega\right)+\kappa+\frac{\kappa_{s}}{2}\right]+g^{2}}
\end{aligned}
$$

where $g$ represents the coupling constant. $\frac{\gamma}{2}$ is the $X^{-}$dipole decay rate. $\kappa$ and $\kappa_{s} / 2$ are the cavity field decay rate into the input and output modes, and the leaky rate, respectively [98]. We can obtain the reflection and transmission coefficients in the approximation of weak excitation with $\omega_{c}=\omega_{X^{-}}=\omega_{0}$ and $g=0$ as:

$$
\begin{aligned}
& r_{0}(\omega)=\frac{i\left(\omega_{0}-\omega\right)+\frac{\kappa_{s}}{2}}{i\left(\omega_{0}-\omega\right)+\frac{\kappa_{s}}{2}+\kappa} \\
& t_{0}(\omega)=\frac{-\kappa}{i\left(\omega_{0}-\omega\right)+\frac{\kappa_{s}}{2}+\kappa}
\end{aligned}
$$

Here, the $\omega_{0}, \omega_{c}$ and $\omega_{X^{-}}$are the frequencies of the input photon, cavity mode and the spin-dependent optical transition, respectively. If we choose $\omega_{0}=\omega$, the reflection coefficient, $|r(\omega)| \approx 1$, and the transmission coefficient, $\left|t_{0}\right|(\omega) \approx 1$. Therefore, the transmission and reflection operators can be rewritten as:

$$
\begin{aligned}
\hat{t}(\omega)= & t_{0}(\omega)(|R\rangle\langle R|\otimes| \uparrow\rangle\langle\uparrow|+| L\rangle\langle L|\otimes| \downarrow\rangle\langle\downarrow|) \\
& +t(\omega)(|R\rangle\langle R|\otimes| \downarrow\rangle\langle\downarrow|+| L\rangle\langle L|\otimes| \uparrow\rangle\langle\uparrow|) \\
\hat{r}(\omega)= & r_{0}(\omega)(|R\rangle\langle R|\otimes| \uparrow\rangle\langle\uparrow|+| L\rangle\langle L|\otimes| \downarrow\rangle\langle\downarrow|) \\
& +r(\omega)(|R\rangle\langle R|\otimes| \downarrow\rangle\langle\downarrow|+| L\rangle\langle L|\otimes| \uparrow\rangle\langle\uparrow|)
\end{aligned}
$$


We can recalculate the success probability in each concentration round as:

$$
P_{K}^{\prime}=\left(\frac{\left|t_{0}(\omega)\right|}{\sqrt{\left|t_{0}(\omega)\right|^{2}+|t(\omega)|^{2}}}\right)^{K-1} \frac{|r(\omega)|}{\sqrt{\left|r_{0}(\omega)\right|^{2}+|r(\omega)|^{2}}} P_{K}
$$

It means that if the protocol is successful in the Kth round, it will have K-1 photons, which are transmitted from the cavity with the transmission coefficient, $\left(\frac{\left|t_{0}(\omega)\right|}{\sqrt{\left|t_{0}(\omega)\right|^{2}+|t(\omega)|^{2}}}\right)^{K-1}$. The total success probability is:

$$
P_{t}=\sum_{K=1}^{\infty} P_{K}^{\prime}
$$

In Figure 23, we calculate the total success probability in both the ideal case with no leakage and with $\kappa_{s}=0.5 \kappa, g=0.5 \kappa$ and $\gamma=0.5 \kappa$. We let $\mathrm{K}=5$ as a good approximation. If the photon should pass through two cavities one time, as shown in [101], the total success probability decreases, as shown in Curve C. The maximally value in Curve $\mathrm{C}$ is about 0.75 .

In a practical operation, we require a long coherent time of the quantum dot and the strong coupling of the quantum dot with the cavity to ensure that the photon can be completely coupled with quantum dot. The current experiment showed that the coherence time of the GaAs- or InAs-based quantum dots is long enough [99]. Interestingly, from Equation (150), the state of the spin does not flip after the photon passes through the cavity. It leads this ECP have the great advantage that the photon loss, and imperfect detection does not affect the fidelity of the protocol.

\section{Discussion and Conclusion}

Thus far, we have briefly explained some ECPs with nonlinear optics and the ECPs encoded in other solid qubits. Most ECPs described are different from the traditional ECPs. In the traditional ECPs, they all require two pairs of partially entangled states to complete the protocol. After performing the protocol, the success probability of obtaining one pair of partially entangled states is $2|\alpha \beta|^{2}$. Certainly, this kind of ECP can be easily extended to the multipartite GHZ state. In this review, we mainly described another way of entanglement concentration, i.e., the ECPs assisted with only single photons. This kind of ECPs have several advantages: First, they can reach the same success probability, but only require one pair of partially entangled states. Therefore, they are optimal. Second, only one of the parities, say Alice, needs to perform the whole protocol, and leads Bob do nothing for Bell-state concentration. It is more useful for concentrating the partially entangled GHZ state. In the previous ECPs, all of the parties should measure their particles. This kind of ECP can save much practical operations during the experiments. Moreover, in traditional ECPs, after the measurement, all of the parties should check their measurement results to decide their remaining maximally entangled states, using classical communications. In these ECPs, only Alice needs to ask all the other parties to retain or discard their particles. It is very economical.

On the other hand, the single photon can be used as an assistant to complete the concentration of the arbitrary partially entangled $\mathrm{W}$ state. In the previous works of concentration of the partially entangled $\mathrm{W}$ state, one cannot complete such task. One can only concentrate some special types of partially entangled $\mathrm{W}$ states. From the above discussion, using the cross-Kerr nonlinearity, the ECPs for the W state can also be repeated to reach a higher success probability. 
The key element for entanglement concentration is the parity check gate. In this way, it is similar to the traditional ECPs. In the optical system, the parity check can be constructed by PBS or cross-Kerr nonlinearity. However, using PBS, one cannot realize a complete parity check gate. One can only obtain the even parity states, $|H H\rangle$ and $|V V\rangle$, and lead the odd parity states, $|H V\rangle$ and $|V H\rangle$, in the different spatial modes. The QND constructed by cross-Kerr nonlinearity acts in the same role as the parity check gate. By measuring the phase shift of the coherent state, one can decide the parity of the two photons. In this review, we have described several QNDs, such as Figure 1, Figure 3, Figures 11 and 15. The QNDs shown in Figures 1 and 3 are used to make the parity check for the polarization degree of freedom, and Figures 11 and 15 are used to make the parity check for spatial modes. The QND in Figure 3 is a little different from Figure 1. To realize a complete parity check, in Figure 1, we should require two different kinds of Kerr material. The one is to generate $\theta$ phase shift, and the other is to generate $-\theta$ phase shift. We also should make the homodyne measurement to make the $\pm \theta$ unable to be distinguished. In Figure 3, we should resort the suitable Kerr material to obtain the $\pi$ phase shift. In the current experiment condition, large phase shift cannot be easily obtained. If we adopt the QND in Figure 3, an effective way is to use the coherent rotation by $\theta$ [54,60]. After performing the coherent rotation, the $\left|\alpha e^{i 2 \theta}\right\rangle$ becomes $\left|\alpha e^{i \theta}\right\rangle$, and $|\alpha\rangle$ becomes $\left|\alpha e^{-i \theta}\right\rangle$. The $\left|\alpha e^{ \pm i \theta}\right\rangle$ cannot be distinguished in a general homodyne measurement. In the electronic system, the PBS and charge detection can also act in the role of the complete parity check gate. It is similar to the quantum dot and optical microcavities systems, as shown in Section 4.3. In Section 4.3, one can judge the parity of the two spin states according to the different spatial mode of the photons.

The cross-Kerr nonlinearity provides us a powerful tool for realizing the entanglement concentration. However, we should acknowledge that it is still a quite-controversial topic to have a clean cross-Kerr-nonlinearity. Shapiro and Razavi performed a detailed analysis and suggested that, in fact, the QND scheme cannot function under realistic conditions [103,104]. Moreover, there has not been any experimental demonstration of schemes making use of the gate. Gea-Banacloche also showed that large shifts via the giant Kerr effect with a single-photon wave packet are impossible with current technology [105]. On the other hand, He et al. have discussed the cross-Kerr nonlinearity between continuous-mode coherent states and single photons, and their work constitutes significant progress in making the treatment of coherent state and single-photon interactions more realistic [106]. With the help of weak measurement, it is possible to amplify a cross-Kerr phase shift to an observable value, as discussed by Feizpour et al.. The phase shift is much larger than the intrinsic magnitude of the single-photon-level nonlinearity[107]. Recently, Zhu and Huang also showed that giant cross-Kerr nonlinearities can be obtained with nearly vanishing optical absorption, investigating the linear and nonlinear propagation of probe and signal pulses, which are coupled in a double-quantum-well structure with a four-level, double-type configuration [108].

The experiment of the ECPs based on the QND depends on the realization of the cross-Kerr nonlinearity. It is not an easy task in the current experimental condition. However, the ECPs with linear optics in this review can be completed, such as the ECP assisted with a single photon, described in Section 2.2, the ECP for the W state in the polarization degree of freedom and the spatial modes degree of freedom. We take the ECP in Section 2.2, for example. As shown in Figure 6, we need to prepare one pair of partially entangled states and a single photon. It can be easily realized with the 
SPDC source. Actually, preparing both the one pair of polarization entanglement and a single polarized photon has been realized in the early work of experimental teleportation [109]. We first prepare two pairs of partially entangled states, like [35]. Then, we trace over one of the photons in the second pair to prepare the single photon.

Certainly, some other ECPs were proposed [110-118]. For example, the ECPs for atoms were proposed [110]. Recently, other ECPs, both for the Bell state and W state, were proposed. In 2012, Deng proposed an ECP with some special polarized photons $\frac{1}{\sqrt{2}}(|H\rangle+|V\rangle)$ [111]. With the help of cross-Kerr nonlinearity, the ECPs for the $\mathrm{W}$ state were also proposed $[112,113]$. Zhou also proposed the ECP for the W state with the help of charge detection [114].

\section{Acknowledgments}

This work is supported by the National Natural Science Foundation of China under Grant No. 11104159, the Open Research Fund Program of the State Key Laboratory of Low-Dimensional Quantum Physics, Tsinghua University, the Open Research Fund Program of National Laboratory of Solid State Microstructures under Grant No. M25022, Nanjing University, the open research fund of Key Lab of Broadband Wireless Communication and Sensor Network Technology, Nanjing University of Posts and Telecommunications, Ministry of Education (No. NYKL201303), and the Project Funded by the Priority Academic Program Development of Jiangsu Higher Education Institutions.

\section{References}

1. Nielsen, M.A.; Chuang, I.L. Quantum Computation and Quantum Information; Cambridge University Press: Cambridge, UK, 2000.

2. Gisin, N.; Ribordy, G.; Tittel, W.; Zbinden, H. Quantum cryptography. Rev. Mod. Phys. 2002, 74, 145-195.

3. Bennett, C.H.; Brassard, G.; Crepeau, C.; Jozsa, R.; Peres, A.; Wootters, W.K. Teleporting an unknown quantum state via dual classical and Einstein-Podolsky-Rosen channels. Phys. Rev. Lett. 1993, 70, 1895-1899.

4. Ekert, A.K. Quantum cryptography based on Bell's theorem. Phys. Rev. Lett. 1991, 67, 661-663.

5. Bennett, C.H.; Brassard, G.; Mermin, N.D. Quantum cryptography without Bell's theorem. Phys. Rev. Lett. 1992, 68, 557-559.

6. Bennett, C.H.; Brassard, G. Quantum Cryptography: Public Key Distribution and Coin Tossing. In Proceedings of IEEE international Conference on Computers, Systems and Signal Processing, Bangalore, India, 10-19 December 1984; pp. 175-179.

7. Long, G.L.; Liu, X.S. Theoretically efficient high-capacity quantum-key-distribution scheme. Phys. Rev. A 2002, 65, 032302.

8. Deng, F.G.; Long, G.L.; Liu, X.S. Two-step quantum direct communication protocol using the Einstein-Podolsky-Rosen pair block. Phys. Rev. A 2003, 68, 042317.

9. Wang, C.; Deng, F.G.; Li, Y.S.; Liu, X.S.; Long, G.L. Quantum secure direct communication with high-dimension quantum superdense coding. Phys. Rev. A 2005, 71, 044305. 
10. Hillery, M.; Bužek, V.; Berthiaume, A. Quantum secret sharing. Phys. Rev. A 1999, 59, 1829-1834.

11. Karlsson, A.; Koashi, M.; Imoto, N. Quantum entanglement for secret sharing and secret splitting. Phys. Rev. A 1999, 59, 162-168.

12. Xiao, L.; Long, G.L.; Deng, F.G.; Pan, J.W. Efficient multiparty quantum-secret-sharing schemes. Phys. Rev. A 2004, 69, 052307.

13. Briegel, H.J.; Dür, W.; Cirac, J.I.; Zoller, P. Quantum repeaters: The role of imperfect local operations in quantum communication. Phys. Rev. Lett. 1998, 81, 5932-5935.

14. Simon, C.; de Riedmatten, H.; Afzelius, M.; Sangouard, N.; Zbinden, H.; Gisin, N. Quantum repeaters with photon pair sources and multimode memories. Phys. Rev. Lett. 2007, 98, 190503.

15. Sangouard, N.; Simon, C.; de Riedmatten, H.; Gisin, N. Quantum repeaters based on atomic ensembles and linear optics. Rev. Mod. Phys. 2011, 83, 33-80.

16. Duan, L.M.; Lukin, M.D.; Cirac, J.T.; Zoller, P. Long-distance quantum communication with atomic ensembles and linear optics. Nature 2001, 414, 413-418.

17. Thew, R.T.; Munro, W.J. Entanglement manipulation and concentration. Phys. Rev. A 2001, 63, 030302(R).

18. Thew, R.T.; Munro, W.J. Mixed state entanglement: Manipulating polarization-entangled photons. Phys. Rev. A 2001, 64, 022320.

19. Bennett, C.H.; Brassard, G.; Popescu, S.; Schumacher, B.; Smolin, J.A.; Wootters, W.K. Purification of noisy entanglement and faithful teleportation via noisy channels. Phys. Rev. Lett. 1996, 76, 722-725.

20. Pan, J.W.; Simon, C.; Zellinger, A. Entanglement purification for quantum communication. Nature 2001, 410, 1067-1070.

21. Simon, C.; Pan, J.W. Polarization entanglement purification using spatial entanglement. Phys. Rev. Lett. 2002, 89, 257901.

22. Sheng, Y.B.; Deng, F.G.; Zhou, H.Y. Efficient polarization-entanglement purification based on parametric down-conversion sources with cross-Kerr nonlinearity. Phys. Rev. A 2008, 77, 042308.

23. Sheng, Y.B.; Deng, F.G. Deterministic entanglement purification and complete nonlocal Bell-state analysis with hyperentanglement. Phys. Rev. A 2010, 81, 032307.

24. Sheng, Y.B.; Deng, F.G. One-step deterministic polarization-entanglement purification using spatial entanglement. Phys. Rev. A 2010, 82, 044305.

25. Deng, F.G. One-step error correction for multipartite polarization entanglement. Phys. Rev. A 2011, 83, 062316.

26. Deng, F.G. Efficient multipartite entanglement purification with the entanglement link from a subspace. Phys. Rev. A 2011, 84, 052312.

27. Li, X.H. Deterministic polarization-entanglement purification using spatial entanglement. Phys. Rev. A 2010, 82, 044304.

28. Cao, C.; Wang, C.; He, L.Y.; Zhang, R. Atomic entanglement purification and concentration using coherent state input-output process in low-Q cavity QED regime. Opt. Exp. 2013, 21, 4093-4105.

29. Wang, C.; Zhang, Y.; Zhang, R. Entanglement purification based on hybrid entangled state using quantum-dot and microcavity coupled system. Opt. Exp. 2011, 19, 25685-25695. 
30. Bennett, C.H.; Bernstein, H.J.; Popescu, S.; Schumacher, B. Concentrating partial entanglement by local operations. Phys. Rev. A 1996, 53, 2046-2052.

31. Bose, S.; Vedral, V.; Knight, P.L. Purification via entanglement swapping and conserved entanglement. Phys. Rev. A 1999, 60, 194-197.

32. Shi, B.S.; Jiang, Y.K.; Guo, G.C. Optimal entanglement purification via entanglement swapping. Phys. Rev. A 2000, 62, 054301.

33. Zhao, Z.; Pan, J.W.; Zhan, M.S. Practical scheme for entanglement concentration. Phys. Rev. A 2001, 64, 014301.

34. Yamamoto, T.; Koashi, M.; Imoto, N. Concentration and purification scheme for two partially entangled photon pairs. Phys. Rev. A 2001, 64, 012304.

35. Zhao, Z.; Yang, T.; Chen, Y.A.; Zhang, A.N.; Pan, J.W. Experimental realization of entanglement concentration and a quantum repeater. Phys. Rev. Lett. 2003, 90, 207901.

36. Yamamoto, T.; Koashi, M.; Ozdemir, S.K.; Imoto, N. Experimental extraction of an entangled photon pair from two identically decohered pairs. Nature 2003, 421, 343-346.

37. Wang, X.B.; Heng, F. Entanglement concentration by ordinary linear optical devices without postselection. Phys. Rev. A 2003, 68, 060302.

38. Boileau, J.-C.; Gottesman, D.; Laflamme, R.; Poulin, D.; Spekkens, R.W. Robust polarization-based quantum key distribution over a collective-noise channel. Phys. Rev. lett. 2004, 92, 017901.

39. Yamamoto, T.; Shimamura, J.; Özdemir, S.K.; Koashi, M.; Imoto, N. Faithful qubit distribution assisted by one additional qubit against collective noise. Phys. Rev. lett. 2005, 95, 040503.

40. Li, X.H.; Deng, F.G.; Zhou, H.Y. Faithful qubit transmission against collective noise without ancillary qubits. Appl. Phys. Lett. 2007, 91, 144101.

41. Deng, F.G.; Li, X.H.; Deng, F.G. Passively self-error-rejecting qubit transmission over a collective-noise channel. Quant. Inf. Comput. 2011, 91, 913-924.

42. Wilde, M.M. From Classical to Quantum Shannon Theory; Cambridge University Press: Cambridge, UK, 2012; in press.

43. Nemoto, K.; Munro, W.J. Nearly deterministic linear optical controlled-not gate. Phys. Rev. Lett. 2004, 93, 250502.

44. Lin, Q.; Li, J. Quantum control gates with weak cross-Kerr nonlinearity. Phys. Rev. A 2009, 79, 022301.

45. Munro, W.J.; Nemoto, K.; Spiller, T.P. Weak nonlinearities: A new route to optical quantum computation. New J. Phys. 2005, doi:10.1088/1367-2630/7/1/137.

46. Barrett, S.D.; Kok, P.; Nemoto, K.; Beausoleil, R.G.; Munro, W.J.; Spiller, T.P. Symmetry analyzer for nondestructive Bell-state detection using weak nonlinearities. Phys. Rev. A 2005, 71, 060302(R).

47. Kurucz, Z.; Adam, P. Preparable ensembles for remote state preparation. J. Opt. B 2005, 7, 135-140.

48. Sheng, Y.B.; Deng, F.G.; Long, G.L. Complete hyperentangled-Bell-state analysis for quantum communication. Phys. Rev. A 2010, 82, 032318. 
49. Lin, Q.; He, B. Single-photon logic gates using minimal resources. Phys. Rev. A 2009, $80,042310$.

50. He, B.; Ren, Y.; Bergou, J.A. Creation of high-quality long-distance entanglement with flexible resources. Phys. Rev. A 2009, 79, 052323.

51. Munro, W.J.; Nemoto, K.; Beausoleil, R.G.; Spiller, T.P. High-efficiency quantum-nondemolition single-photon-number-resolving detector. Phys. Rev. A 2005, 71, 033819.

52. Sheng, Y.B.; Deng, F.G. Efficient quantum entanglement distribution over an arbitrary collective-noise channel. Phys. Rev. A 2010, 81, 042332.

53. Sheng, Y.B.; Deng, F.G.; Zhou, H.Y. Nonlocal entanglement concentration scheme for partially entangled multipartite systems with nonlinear optics. Phys. Rev. A 2008, 77, 062325.

54. Sheng, Y.B.; Zhou, L.; Zhao, S.M.; Zheng, B.Y. Efficient single-photon-assisted entanglement concentration for partially entangled photon pairs. Phys. Rev. A 2012, 85, 012307.

55. Sheng, Y.B.; Deng, F.G.; Zhou, H.Y. Single-photon entanglement concentration for long distance quantum communication. Quant. Inf. Comput. 2010, 10, 272-281.

56. Munro, W.J.; Nemoto, K.; Spiller, T.P.; Beausoleil, R.G.; Kok, P.; Barrett, S.D. Optical quantum information processing utilizing weak nonlinearities: A little goes a long way. Proc. SPIE 2005, 5893, Quantum Communications and Quantum Imaging III, 58930I, doi:10.1117/12.613614.

57. Cao, Z.L.; Yang, M. Entanglement distillation for three-particle W class states. J. Phys. B 2003, 36, 4245-4253.

58. Zhang, L.H.; Yang, M.; Cao, Z.L. Entanglement concentration for unknown W class states. Phys. Stat. Mech. Appl. 2007, 374, 611-616.

59. Yildiz, A. Optimal distillation of three-qubit W states. Phys. Rev. A 2010, 82, 012317.

60. Sheng, Y.B.; Zhou, L.; Zhao, S.M. Efficient two-step entanglement concentration for arbitrary W states. Phys. Rev. A 2012, 85, 044305.

61. Zhou, L.; Sheng, Y.B.; Cheng, W.W.; Gong, L.Y.; Zhao, S.M. Efficient entanglement concentration for arbitrary single-photon multimode W state. J. Opt. Soc. Am. B 2013, 30, 71-78.

62. Furusawa, A.; Søensen, J.L.; Braunstein, S.L.; Fuchs, C.A.; Kimble, H.J.; Polzik, E.S. Unconditional quantum teleportation. Science 1998, 282, 706-709.

63. Jing, J.; Zhang, J.; Yan, Y.; Zhao, F.; Xie, C.; Peng, K. Experimental demonstration of tripartite entanglement and controlled dense coding for continuous variables. Phys. Rev. Lett. 2003, 90, 167903.

64. Aoki, T.; Takey, N.; Yonezawa, H.; Wakui, K.; Hiraoka, T.; Furusawa, A.; van Loock, P. Experimental creation of a fully inseparable tripartite continuous-variable state. Phys. Rev. Lett. 2003, 91, 080404.

65. Göckl, O.; Lorenz, S.; Marquardt, C.; Heersink, J.; Brownnutt, M.; Silberhorn, C.; Pan, Q.; van Loock, P.; Korolkova, N.; Leuchs, G. Experiment towards continuous-variable entanglement swapping: Highly correlated four-partite quantum state. Phys. Rev. A 2003, 68, 012319.

66. Sen(De), A.; Sen, U.; Wieśniak, M.; Kaszlikowski, D.; Żukowski, M. Multiqubit W states lead to stronger nonclassicality than Greenberger-Horne-Zeilinger states. Phys. Rev. A 2003, 68, 062306. 
67. Dür, W.; Vidal, G.; Cirac, J.I. Three qubits can be entangled in two inequivalent ways. Phys. Rev. A 2000, 62, 062314.

68. Chaves, R.; Davidovich, L. Robustness of entanglement as a resource. Phys. Rev. A 2010, 82, 052308 .

69. Heaney, L.; Cabello, A.; Santos, M.F.; Vedral, V. Extreme nonlocality with one photon. New J. Phys. 2011, 13, 053054-053065.

70. Gottesman, D.; Jennewein, T.; Croke, S. Longer-Baseline Telescopes using quantum repeaters. Phys. Rev. Lett. 2012, 109, 070503.

71. Osorio, C.I.; Bruno, N.; Sangouard, N.; Zbinden, H.; Gisin, N.; Thew, R.T. Heralded photon amplification for quantum communication. Phys. Rev. A 2012, 86, 023815.

72. Dowling, J.P. Quantum optical metrology-the lowdown on high-N00N states. Contemp. Phys. 2008, 49, 125-143.

73. Huelga, S.F.; Macchiavello, C.; Pellizzari, T.; Ekert, A.K. Improvement of frequency standards with quantum entanglement. Phys. Rev. Lett. 1997, 79, 3865-3868.

74. Mitchell, M.W.; Lundeen, J.S.; Steinberg, A.M. Super-resolving phase measurements with a multiphoton entangled state. Nature 2004, 429, 161-164.

75. Walther, P.; Pan, J.W.; Aspelmeyer, M.; Ursin, R.; Gasparoni, S.; Zeilinger, A. De Broglie wavelength of a non-local four-photon state. Natue 2004, 429, 158-161.

76. Nagata, T.; Okamoto, R.; ÓBrien, J.L.; Sasaki, K.; Takeuchi, S. Beating the standard quantum limit with four-entangled photons. Science 2007, 316, 726-729.

77. Mermin, N.D. Extreme quantum entanglement in a superposition of macroscopically distinct states. Phys. Rev. Lett. 1990, 65, 1838-1841.

78. Boto, A.N.; Kok, P.; Abrams, D.S.; Braunstein, S.L.; Williams, C.P.; Dowling, J.P. Quantum interferometric optical lithography: Exploiting entanglement to beat the diffraction limit. Phys. Rev. Lett. 2000, 85, 2733-2736.

79. Bollinger, J.J.; Itano, W.M.; Wineland, D.J.; Heinzen, D.J. Optimal frequency measurements with maximally correlated states. Phys. Rev. A 1996, 54, R4649-R4652.

80. D'Angelo, M.; Chekhova, M.V.; Shih, Y. Two-photon diffraction and quantum lithography. Phys. Rev. Lett. 2001, 87, 013602.

81. Sun, F.W.; Ou, Z.Y.; Guo, G.C. Simple proof of fault tolerance in the graph-state model. Phys. Rev. A 2006, 73, 032308.

82. Liu, B.; Ou, Z.Y. Feasibility of Bell tests with the W state. Phys. Rev. A 2010, 81, 033823.

83. Zhou, L.; Sheng, Y.B.; Cheng, W.W.; Gong, L.Y.; Zhao, S.M. Efficient entanglement concentration for arbitrary less-entangled NOON states. Quant. Inf. Process. 2013, 12, 1307-1320.

84. Beenakker, C.W.J.; DiVincenzo, D.P.; Emary, C.; Kindermann, M. Charge detection enables free-electron quantum computation. Phys. Rev. Lett. 2004, 93, 020501.

85. Terhal, B.M.; DiVincenzo, D.P. Classical simulation of noninteracting-fermion quantum circuits. Phys. Rev. A 2002, 65, 032325.

86. Zhang, X.L.; Feng, M.; Gao, K.L. Cluster-state preparation and multipartite entanglement analyzer with fermions. Phys. Rev. A 2006, 73, 014301. 
87. Feng, X.L.; Kwek, L.C.; Oh, C.H. Electronic entanglement purification scheme enhanced by charge detections. Phys. Rev. A 2005, 71, 064301.

88. Li, T.; Ren, B.C.; Wei, H.R.; Hua, M.; Deng, F.G. High-efficiency multipartite entanglement purification of electron-spin states with charge detection. Quant. Inf. Process. 2013, 12, 855-876.

89. Sheng, Y.B.; Deng, F.G.; Zhou, H.Y. Multipartite electronic entanglement purification with charge detection. Phys. Lett. A 2011, 375, 396-400.

90. Sheng, Y.B.; Zhou, L.; Cheng, W.W.; Gong, L.Y.; Zhao, S.M. Efficient electronic entanglement concentration assisted with single mobile electron. Chin. Phys. B 2013, accepted.

91. Wang, C.; Zhang, R.; Zhang, Y.; Ma, H.Q. Multipartite electronic entanglement purification using quantum-dot spin and microcavity system. Quant. Inf. Process. 2013, 12, 525-536.

92. Trauzettel, B.; Jordan, A.N.; Beenakker, C.W.; Büttiker, M. Parity meter for charge qubits: An efficient quantum entangler. Phys. Rev. B 2006, 73, 235331.

93. Ionicioiu, R. Entangling spins by measuring charge: A parity-gate toolbox. Phys. Rev. A 2007, 75, 032339.

94. Sheng, Y.B.; Deng, F.G.; Zhou, H.Y. Efficient polarization entanglement concentration for electrons with charge detection. Phys. Lett. A 2009, 373, 1823-1825.

95. Waks, E.; Vuckovic, J. Dipole induced transparency in drop-filter cavity-waveguide systems. Phys. Rev. Lett. 2006, 96, 153601.

96. Bonato, C.; Haupt, F.; Oemrawsingh, S.S.R.; Gudat, J.; Ding, D.; van Exter, M.P.; Bouwmeester, D. CNOT and Bell-state analysis in the weak-coupling cavity QED regime. Phys. Rev. Lett. 2010, 104, 160503.

97. Hu, C.Y.; Munro, W.J.; Rarity, J.G. Deterministic photon entangler using a charged quantum dot inside a microcavity. Phys. Rev. B 2008, 78, 125318.

98. Hu, C.Y.; Munro, W.J.; ÓBrien, J.L.; Rarity, J.G. Proposed entanglement beam splitter using a quantum- dot spin in a double-sided optical microcavity. Phys. Rev. B 2009, 80, 205326.

99. Xu, X.; Yao, W.; Sun, B.; Steel, D.G.; Bracker, A.S.; Gammon, D.; Sham, L.J. A Jurassic ceratosaur from China helps clarify avian digital homologies. Nature 2009, 459, 940.

100. Wang, C.; Zhang, Y.; Jin, G.S. Entanglement purification and concentration of electron-spin entangled states using quantum-dot spins in optical microcavities. Phys. Rev. A 2011, 84, 032307.

101. Wang, C. Efficient entanglement concentration for partially entangled electrons using a quantum-dot and microcavity coupled system. Phys. Rev. A 2012, 86, 012323.

102. Sheng, Y.B.; Zhou, L.; Wang, L.; Zhao, S.M. Efficient entanglement concentration for quantum dot and optical microcavities systems. Quant. Inf. Process. 2013, 12, 1885-1895.

103. Shapiro, J.H. Single-photon Kerr nonlinearities do not help quantum computation. Phys. Rev. A 2006, 73, 062305.

104. Shapiro, J.H.; Razavi, M. Continuous-time cross-phase modulation and quantum computation. New J. Phys. 2007, doi:10.1088/1367-2630/9/1/016.

105. Gea-Banacloche, J. Impossibility of large phase shifts via the giant Kerr effect with single-photon wave packets. Phys. Rev. A 2010, 81, 043823. 
106. He, B.; Lin, Q.; Simon, C. Cross-Kerr nonlinearity between continuous-mode coherent states and single photons. Phys. Rev. A 2011, 83, 053826.

107. Feizpour, A.; Xing, X.; Steinberg, A.M. Amplifying single-photon nonlinearity using weak measurements. Phys. Rev. Lett. 2011, 107, 133603.

108. Zhu, C.; Huang, G. Giant Kerr nonlinearity, controlled entangled photons and polarization phase gates in coupled quantum-well structures. Opt. Express 2011, 19, 23364-23376 .

109. Bouwmeester, D.; Pan, J.W.; Mattle, K.; Eibl, M.; Weinfurter, H.; Zeilinger, A. Experimental quantum teleportation. Nature 1997, 390, 575-579.

110. Peng, Z.H.; Zou, J.; Liu, X.J.; Xiao, Y.J.; Kuang, L.M. Atomic and photonic entanglement concentration via photonic Faraday rotation. Phys. Rev. A 2012, 86, 034305.

111. Deng, F.G. Optimal nonlocal multipartite entanglement concentration based on projection measurements. Phys. Rev. A 2012, 85, 022311.

112. Du, F.F.; Li, T.; Ren, B.C.; Wei, H.R.; Deng, F.G. Single-photon-assisted entanglement concentration of a multi-photon system in a partially entangled $\mathrm{W}$ state with weak cross-Kerr nonlinearity. J. Opt. Soc. Am. B 2012, 29, 1399-1405.

113. Gu, B. Single-photon-assisted entanglement concentration of partially entangled multiphoton W states with linear optics. J. Opt. Soc. Am. B 2012, 29, 1685-1689.

114. Zhou, L. Efficient entanglement concentration for electron-spin W state with the charge detection. Quant. Inf. Process. 2013, 12, 2087-2101.

115. Sheng, Y.B.; Zhou, L. Efficient W-state entanglement concentration using quantum-dot and optical microcavities. J. Opt. Soc. Am. B 2013, 30, 678-686.

116. Wang, H.F.; Zhang, S.; Yeon, K.H. Linear-optics-based entanglement concentration of unknown partially entangled three photon W states. J. Opt. Soc. Am. B 2010, 27, 2159-2164.

117. Xiong, W.; Ye, L. Schemes for entanglement concentration of two unknown partially entangled states with cross-Kerr nonlinearity. J. Opt. Soc. Am. B 2011, 28, 2030-2037.

118. Wang, T.J.; Long, G.L. Entanglement concentration for arbitrary unknown less-entangled three-photon W states with linear optics. J. Opt. Soc. Am. B 2013, 30, 1069-1076.

(c) 2013 by the authors; licensee MDPI, Basel, Switzerland. This article is an open access article distributed under the terms and conditions of the Creative Commons Attribution license (http://creativecommons.org/licenses/by/3.0/). 